\title{
Provenance characteristics of Scandinavian basement terrains: Constraints from detrital zircon ages in modern river sediments
}

\author{
Andrew Morton ${ }^{\mathrm{a}, \mathrm{b}, *}$, Mark Fanning ${ }^{\mathrm{c}}$, Paul Milner ${ }^{\mathrm{d}, 1}$

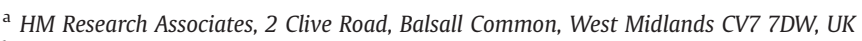 \\ b CASP, Department of Earth Sciences, University of Cambridge, 181a Huntingdon Road, Cambridge CB3 ODH, UK \\ ${ }^{c}$ Research School of Earth Sciences, The Australian National University, Canberra, ACT 0200, Australia \\ ${ }^{d}$ BP Norge a/s, BP-gården, Godesetdalen 8, P.O. Box 197, 4065 Stavanger, Norway
}

\section{A R T I C L E I N F O}

\section{Article history:}

Received 24 February 2008

Received in revised form 20 June 2008

Accepted 4 July 2008

\section{Keywords:}

Provenance

Zircon ages

Fennoscandian Shield

Caledonian Nappes

Cretaceous

\begin{abstract}
A B S T R A C T
A study of modern river sediments from northern Scandinavia has shown that detrital zircon populations derived from the Fennoscandian Shield are fundamentally different to those derived from the Caledonian Nappe Domain. The zircon age spectra derived from the Fennoscandian Shield are essentially unimodal or bimodal, whereas the Caledonian Nappe Domain spectra are much more diverse, showing multiple peaks and a wider range of ages. The greater diversity of the Caledonian Domain zircon populations compared with those from the Fennoscandian Shield reflects the fundamentally different nature of the source regions. The Caledonian Nappe Domain largely comprises metasedimentary rocks, which were themselves derived from the variety of precursor sources available at the time, mainly situated in Baltica but with Laurentian influences in the Uppermost Allochthon. By contrast, the Fennoscandian Shield zircons were largely derived from granitoid rocks formed during specific crust-forming events. Thus, the Fennoscandian Shield spectra are typical of first-cycle, basement-derived detritus, whereas the Caledonian Nappe Domain spectra are typical of a multicyclic source. The study suggests that zircon age spectra, when combined with mineralogical or petrographic data, can help to distinguish first-cycle from polycyclic sediment, with complex zircon age spectra being likely to diagnose polycyclic sources, and simple spectra more likely to indicate first-cycle sediment. Comparison between the zircons in Scandinavian rivers and Cretaceous sandstones in the Norwegian Sea support previous interpretations of provenance data that indicate a non-Scandinavian source (East Greenland) for some of the Cretaceous sandstones.
\end{abstract}

(c) 2008 Elsevier B.V. All rights reserved.

\section{Introduction}

In recent years, sediment provenance studies have been revolutionised by technological developments that enable single-grain radiometric dating of some components of detrital grain populations, for example U-Pb dating of zircon (e.g. Ireland, 1992) and monazite (e.g. Evans et al., 2001), or Ar-Ar dating of hornblende (Hemming et al., 2000) and mica (e.g. Von Eynatten et al., 1996). Radiometric data provide constraints on the geological history of sediment source regions. These supplement lithological and mineralogical information acquired by more traditional methods, such as petrography, heavy mineral analysis or whole-rock geochemistry, and a combined approach that integrates radiometric, mineralogical and geochemical data provides a more

\footnotetext{
* Corresponding author. HM Research Associates, 2 Clive Road, Balsall Common, West Midlands CV7 7DW, UK.

E-mail address: a.c.morton@heavyminerals.fsnet.co.uk (A. Morton).

Current address: Gaz de France Norge AS, Vestre Svanholmen 6, Sandnes, Postboks 242, 4066 Stavanger, Norway.
}

complete and accurate characterisation of sediment provenance (e.g. Von Eynatten and Gaupp, 1999; Morton et al., 2005a).

Single-grain U-Pb dating of detrital zircon, either by sensitive highresolution ion microprobe (SHRIMP: Williams, 1998) or laser ablation inductively-coupled mass spectrometry (LA-ICPMS: Kosler and Sylvester, 2003) has proved especially useful in provenance studies, largely because of the stability of zircon in both weathering and diagenetic regimes (Fedo et al., 2003). Thus, zircon is not liable to destruction during the sedimentary cycle unless it has become metamict. Zircon forms predominantly in felsic-intermediate igneous rocks and highgrade (granulite facies) metamorphic rocks, thereby providing a record of major crust-forming events in the hinterland. Owing to its chemical and mechanical stability, zircon is readily recycled through successive sedimentary cycles. By establishing a direct link between sediment and source, detrital zircon geochronology has major applications in paleogeographic studies, for example establishing the sourcelands for ancient continental margin successions (Cawood et al., 2003), resolving tectonic problems of terrane translation (Mahoney et al., 1999), or constraining intrabasinal sand distribution by identifying sediment input from different entry points (Morton et al., 2005a). 
Most previous detrital zircon studies have been carried out by characterising detrital zircon age spectra, and linking the main peaks in these spectra with known crust-forming events in the hinterland. This approach is valid providing the ages of the zircons in the hinterland are well known through previous radiometric studies. This is generally true, to a greater or lesser extent, for first cycle zircons directly derived from their igneous or metamorphic source, although in more remote areas of the world, ages of basement rocks may be relatively poorly constrained. However, it is generally more difficult to establish the detrital zircon age spectra supplied by terrains dominated by pre-existing sedimentary or metasedimentary rocks, since there are comparatively few datasets available from such lithologies. Even when datasets are available, they are generally based on analysis of a relatively small number of individual samples. Given the likelihood of complex heterogeneity in provenance within pre-existing sedimentary or metasedimentary successions, such data are unlikely to give a balanced view of the zircon age spectra provided by such terrains.

This paper takes an alternative approach to detrital zircon provenance studies, by analysing zircons in modern river sediments from locations between $62^{\circ}-69^{\circ} \mathrm{N}$ in Norway and Sweden. This region comprises two contrasting crustal domains, the Fennoscandian Shield in the east and the Caledonian Nappe Domain in the west (Fig. 1). The main aim of the study was to compare and contrast the nature of the zircon age populations supplied from these two domains. Subsidiary aims were to establish whether the age

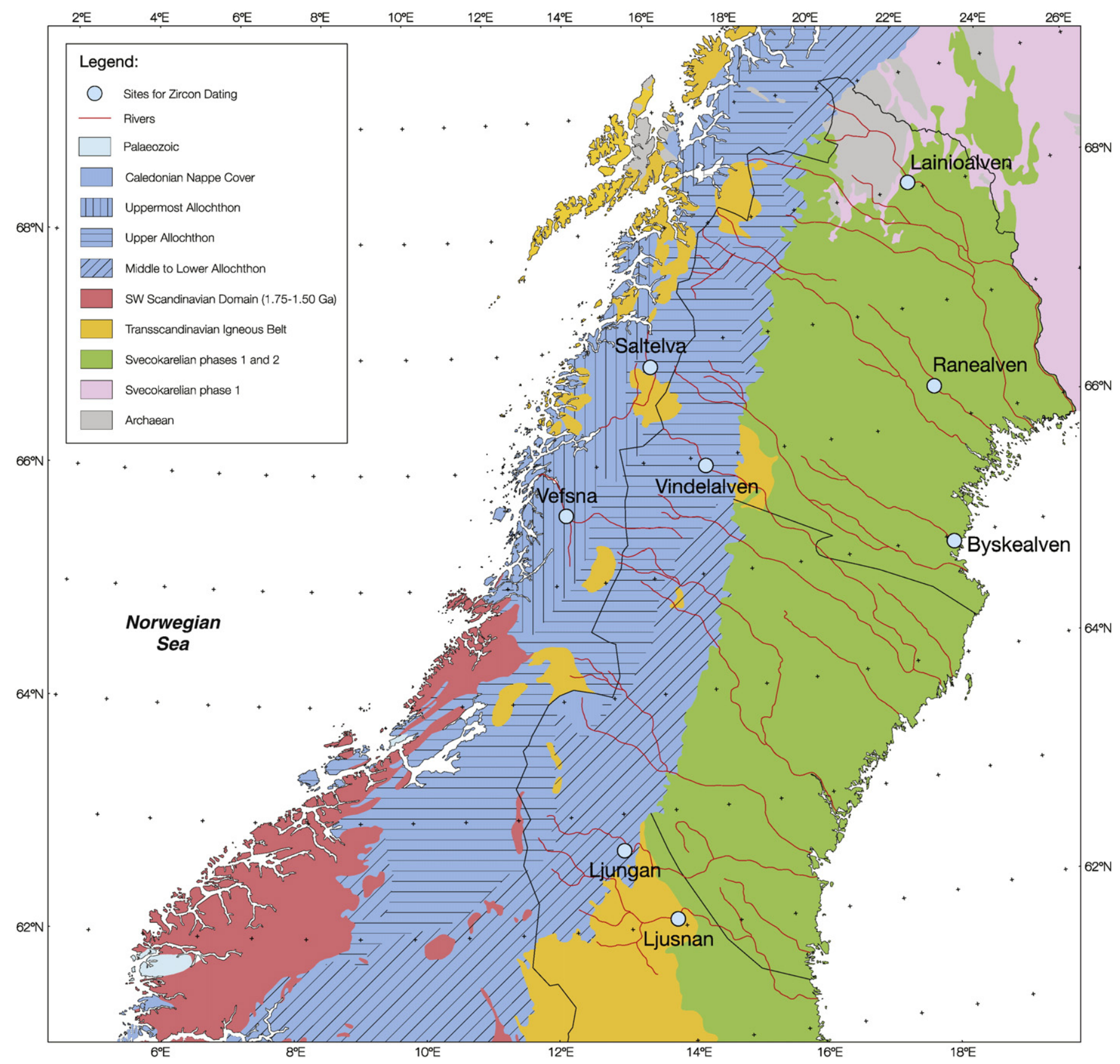

Fig. 1. Generalised geological map of central and northern Scandinavia, showing major rivers and the location of the eight sample sites discussed in this paper. 
structures of the detrital zircon populations can be reconciled with the known ages of the Fennoscandian Shield parent rocks, and to provide broad constraints on the basement rocks that supplied the metasediments of the Caledonian Nappe Domain. The study also provides constraints concerning provenance of hydrocarbon-bearing Cretaceous-Paleocene sandstones in the Norwegian Sea. Some of these sandstones have zircon age populations that suggest derivation from the conjugate East Greenland margin (Fonneland et al., 2004; Morton et al., 2005a,b). However, it is not possible to entirely rule out provenance from Scandinavia, because the zircon age spectra from Caledonian metasediments are poorly known, and because of possible sourcing from the Fennoscandian Shield east of the Caledonian front (Morton et al., 2005a,b). This possibility can be tested directly by comparing Cretaceous-Paleocene zircon ages from sediments offshore mid-Norway with those from the Scandinavian rivers.

\section{Geological setting and sampling strategy}

The Fennoscandian Shield dominantly consists of crystalline rocks that originated in a series of major crust-forming events, with relatively limited metasedimentary cover (Gaal and Gorbatschev, 1987; Koistinen et al., 2001). In northern Sweden, the Fennoscandian Shield largely comprises granitoids and coeval volcanic rocks that formed during Svecokarelian phase 1 (1.90-1.88 Ga) and Svecokarelian phase 2 (1.86-1.75 Ga), together with metasedimentary units (Koistinen et al., 2001). In the northwest of the study area, there is an outcrop of Archaean basement rocks (mainly granitoids, migmatites and grey gneisses, formed between $2.75-2.50 \mathrm{Ga}$ ), overlain by the Lapponian volcanic succession, which consists predominantly of mafic or ultramafic rocks with an upper age limit of c. $2.3 \mathrm{Ga}$ (Gaal and Gorbatschev, 1987; Koistinen et al., 2001). The Trans-Scandinavian Igneous Belt, which is a late-stage component of the Fennoscandian Shield that outcrops in the south of the region (Fig. 1), consists of three magmatic episodes in the Trans-Scandinavian Igneous Belt, TIB1 at 1.81-1.77 Ga, TIB2 at $1.7 \mathrm{Ga}$, and TIB3 at 1.68-1.65 Ga (Larson and Berglund, 1992).

By contrast, the Caledonian Nappe Domain, which comprises a series of thrust sheets (successively, the Lower, Middle, Upper and Uppermost Allochthons) emplaced onto the Fennoscandian Shield during the Early Paleozoic, consists largely of metasedimentary rocks of varying metamorphic grades (Roberts and Gee, 1985), although a wide variety of subordinate lithologies, including granites, ophiolites and basic gneisses, are also present. The rocks comprising the Lower and Middle Allochthons represent shelf and continental rise deposits on the Baltoscandian margin of Baltica, whereas the Upper Allochthon comprises ophiolites of oceanic origin, igneous rocks of magmatic arc affinity, and marginal basin successions within or peripheral to the Iapetus Ocean (Roberts, 2003). The Uppermost Allochthon, which is largely metasedimentary, is believed to have had a Laurentian ancestry (Roberts, 2003; Kirkland et al., 2007). In addition to the thrust sheets, there are outcrops of basement windows within the Caledonian Nappe Domain. These basement windows correlate with Svecokarelian phase 2 granitoids and the oldest Trans-Scandinavian event, TIB1 (Skår, 2002; Rehnström and Corfu, 2004).

The rationale adopted in this paper is to characterise the zircon age spectra supplied by these two strongly contrasting types of sediment source terrain. The geological history of the Fennoscandian Shield is well known, with a long history of geochronological investigations (Gaal and Gorbatschev, 1987; Koistinen et al., 2001). By contrast, comparatively little is known of the ages of detrital zircons in the metasediments of the Caledonian Nappe Domain. The only data in the study area are from the Seve Nappes (Williams and Claesson, 1987), although there are also data from the Kalak Nappe Complex (Kirkland et al., 2007), which lies in northern Norway, outside the area currently under investigation.
Table 1

Location of samples discussed in this study

\begin{tabular}{llll}
\hline river & draining & latitude & longitude \\
\hline Byskeälven & Fennoscandian shield & 6457.460 & 2108.905 \\
Raneälven & Fennoscandian shield & 6618.445 & 2122.012 \\
Lainioälven & Archaean & 6805.386 & 2140.957 \\
Ljusnan & Trans Scandinavian Igneous Belt & 6202.691 & 1449.557 \\
Saltelva & Norwegian Caledonides & 6650.286 & 1520.579 \\
Vefsna & Norwegian Caledonides & 6536.703 & 1318.401 \\
Vindelälven & Swedish Caledonides & 6554.480 & 1618.853 \\
Ljungan & Swedish Caledonides & 6241.010 & 1358.222 \\
\hline
\end{tabular}

Zircon age data have been acquired from eight samples between $62-69^{\circ} \mathrm{N}$ in Norway and Sweden (Fig. 1; Table 1). Four of the samples are from rivers draining the Fennoscandian Shield. One of these (Lainioälven) taps the outcrop of Archaean rocks in the northern part of the area, and two (Byskeälven and Raneälven) drain basement rocks formed in Svecokarelian phases 1 and 2 (Koistinen et al., 2001). The other sample is from the river Ljusnan, which drains the northern part (Dalarna province) of the Trans-Scandinavian Igneous Belt (Larson and Berglund, 1992). The other four samples are from river sediments draining the Caledonian Nappe Domain. One river (Ljungan) drains the Middle and Lower Allochthons, one (Vindelälven) drains the Upper Allochthon, and two (Vefsna and Saltelva) drain the Upper and Uppermost Allochthons. The catchments of Vindelälven, Saltelva and Vesfna either include, or are closely adjacent to, basement windows within the Caledonian Nappe Domain.

\section{Analytical methods}

Zircon concentrates were obtained using standard density and magnetic separation techniques. Arbitrary, and presumed representative, fractions for each sample were poured onto double sided tape, cast into an epoxy resin disk, sectioned and polished. Transmitted and reflected light photomicrographs, together with cathodoluminescence (CL) images, were prepared for all grains. The $\mathrm{U}-\mathrm{Pb}$ analyses were undertaken using SHRIMP I and SHRIMP RG at The Australian National University in Canberra. The procedures employed for zircon U-Pb dating followed Williams (1998) and references therein. The number of scans through the mass stations was limited to four, thereby achieving rapid data acquisition at the expense of some counting precision per analysis. In the first instance, an arbitrary group of 60 zircons were analysed from each sample. Subjectivity in zircon dating was avoided by analysing all zircons encountered during the traverse of the mount, unless the grain showed evidence of being metamict or otherwise structurally compromised as determined from examination of the reflected and transmitted light photomicrographs and CL images. Normalisation of $\mathrm{Pb} / \mathrm{U}$ isotopic ratios was achieved by reference to analyses of the FC1 reference zircon (1099 Ma: ${ }^{206} \mathrm{~Pb} /{ }^{238} \mathrm{U}=0.1589$ : Paces and Miller, 1993). The raw SHRIMP data were processed using SQUID (Ludwig, 2001), with plots generated using Isoplot/Ex (Ludwig, 1999). For zircon areas that are older than approximately $800 \mathrm{Ma}$, the measured ${ }^{206} \mathrm{~Pb} /{ }^{204} \mathrm{~Pb}$ ratios have been used to correct for common $\mathrm{Pb}$ and the radiogenic ${ }^{207} \mathrm{~Pb} /{ }^{206} \mathrm{~Pb}$ ratio used to calculate the preferred age. For zircon areas that are younger than approximately $800 \mathrm{Ma}$, correction for common $\mathrm{Pb}$ was made using the measured ${ }^{207} \mathrm{~Pb} /{ }^{206} \mathrm{~Pb}$ and ratios ${ }^{238} \mathrm{U} /{ }^{206} \mathrm{~Pb}$ ratios, giving a radiogenic ${ }^{206} \mathrm{~Pb} /$ ${ }^{238} \mathrm{U}$ ratio and age following Tera and Wasserburg (1972) as described by Williams (1998). For Neoproterozoic and older zircons, when an analysis is more than $20 \%$ discordant it has been excluded from the relative probability plots. For the younger zircons, the validity of the radiogenic ${ }^{206} \mathrm{~Pb} /{ }^{238} \mathrm{U}$ age has been determined on the basis of a number of factors, including the amount of common $\mathrm{Pb}$ (that is, if the total ${ }^{207} \mathrm{~Pb} /{ }^{206} \mathrm{~Pb}$ ratio deviates significantly from 
Table 2

Summary of SHRIMP U-Pb zircon results for Byskeälven river sand

\begin{tabular}{|c|c|c|c|c|c|c|c|c|c|c|c|c|c|c|c|c|c|c|c|c|c|c|}
\hline \multirow[b]{2}{*}{$\begin{array}{l}\text { Grain. } \\
\text { spot }\end{array}$} & \multirow[b]{2}{*}{$\begin{array}{l}\mathrm{U} \\
(\mathrm{ppm})\end{array}$} & \multirow[b]{2}{*}{$\begin{array}{l}\text { Th } \\
(\mathrm{ppm})\end{array}$} & \multirow[b]{2}{*}{$\mathrm{Th} / \mathrm{U}$} & \multirow[b]{2}{*}{$\begin{array}{l}\mathrm{Pb}^{*} \\
(\mathrm{ppm})\end{array}$} & \multirow[b]{2}{*}{${ }^{204} \mathrm{~Pb} /{ }^{206} \mathrm{~Pb}$} & \multirow[b]{2}{*}{$\mathrm{f}_{206} \%$} & \multicolumn{4}{|l|}{ Total Ratios } & \multicolumn{6}{|c|}{ Radiogenic Ratios } & \multirow[b]{2}{*}{$\mathrm{r}$} & \multicolumn{4}{|l|}{ Age (Ma) } & \multirow[b]{2}{*}{ \% Disc } \\
\hline & & & & & & & ${ }^{238} \mathrm{U} /{ }^{206} \mathrm{~Pb}$ & \pm & ${ }^{207} \mathrm{~Pb} /{ }^{206} \mathrm{~Pb}$ & \pm & ${ }^{206} \mathrm{~Pb} /{ }^{238} \mathrm{U}$ & \pm & ${ }^{207} \mathrm{~Pb} /{ }^{235} \mathrm{U}$ & \pm & ${ }^{207} \mathrm{~Pb} /{ }^{206} \mathrm{~Pb}$ & \pm & & ${ }^{206} \mathrm{~Pb} /{ }^{238} \mathrm{U}$ & \pm & ${ }^{207} \mathrm{~Pb} /{ }^{206} \mathrm{~Pb}$ & \pm & \\
\hline 1.1 & 103 & 70 & 0.68 & 23.3 & 0.000017 & 0.03 & 3.787 & 0.054 & 0.0942 & 0.0010 & 0.2640 & 0.0038 & 3.419 & 0.062 & 0.0939 & 0.0010 & 0.788 & 1510 & 19 & 1507 & 21 & 0 \\
\hline 2.1 & 137 & 31 & 0.23 & 38.4 & 0.000032 & 0.05 & 3.063 & 0.040 & 0.1086 & 0.0009 & 0.3264 & 0.0043 & 4.868 & 0.075 & 0.1082 & 0.0009 & 0.847 & 1821 & 21 & 1769 & 15 & -3 \\
\hline 3.1 & 190 & 56 & 0.29 & 54.1 & 0.000056 & 0.09 & 3.023 & 0.038 & 0.1085 & 0.0008 & 0.3305 & 0.0041 & 4.910 & 0.070 & 0.1077 & 0.0008 & 0.869 & 1841 & 21 & 1762 & 13 & -4 \\
\hline 4.1 & 132 & 47 & 0.35 & 19.9 & - & $<0.01$ & 5.701 & 0.077 & 0.0763 & 0.0013 & 0.1752 & 0.0024 & 1.819 & 0.040 & 0.0753 & 0.0013 & 0.623 & 1041 & 14 & 1077 & 34 & 3 \\
\hline 5.1 & 266 & 57 & 0.22 & 46.7 & 0.000011 & 0.02 & 4.898 & 0.058 & 0.0836 & 0.0007 & 0.2038 & 0.0024 & 2.304 & 0.034 & 0.0820 & 0.0007 & 0.809 & 1196 & 13 & 1246 & 17 & 4 \\
\hline 6.1 & 57 & 38 & 0.68 & 7.8 & 0.000000 & $<0.01$ & 6.251 & 0.112 & 0.0768 & 0.0016 & 0.1588 & 0.0028 & 1.544 & 0.048 & 0.0705 & 0.0018 & 0.573 & 950 & 18 & 943 & 52 & -1 \\
\hline 7.1 & 445 & 120 & 0.27 & 131.3 & 0.000028 & 0.04 & 2.913 & 0.032 & 0.1090 & 0.0005 & 0.3431 & 0.0038 & 5.137 & 0.063 & 0.1086 & 0.0005 & 0.913 & 1902 & 18 & 1776 & 9 & -7 \\
\hline 8.1 & 53 & 37 & 0.70 & 11.2 & - & $<0.01$ & 4.058 & 0.071 & 0.0897 & 0.0015 & 0.2469 & 0.0043 & 3.108 & 0.084 & 0.0913 & 0.0019 & 0.647 & 1422 & 22 & 1453 & 39 & 2 \\
\hline 9.1 & 147 & 41 & 0.28 & 23.3 & 0.000027 & 0.05 & 5.421 & 0.072 & 0.0777 & 0.0009 & 0.1844 & 0.0024 & 1.965 & 0.035 & 0.0773 & 0.0009 & 0.737 & 1091 & 13 & 1129 & 24 & 3 \\
\hline 10.1 & 133 & 29 & 0.22 & 41.9 & 0.000000 & $<0.01$ & 2.735 & 0.037 & 0.1147 & 0.0010 & 0.3657 & 0.0050 & 5.781 & 0.092 & 0.1147 & 0.0010 & 0.854 & 2009 & 23 & 1875 & 15 & -7 \\
\hline 11.1 & 473 & 140 & 0.30 & 126.3 & 0.000035 & 0.05 & 3.219 & 0.035 & 0.1134 & 0.0005 & 0.3105 & 0.0034 & 4.836 & 0.057 & 0.1129 & 0.0005 & 0.920 & 1743 & 17 & 1847 & 8 & 6 \\
\hline 12.1 & 584 & 140 & 0.24 & 145.4 & 0.000014 & 0.02 & 3.453 & 0.037 & 0.1097 & 0.0004 & 0.2896 & 0.0031 & 4.373 & 0.051 & 0.1095 & 0.0005 & 0.929 & 1639 & 16 & 1792 & 8 & 8 \\
\hline 13.1 & 94 & 63 & 0.66 & 16.9 & 0.000074 & 0.12 & 4.798 & 0.069 & 0.0848 & 0.0013 & 0.2082 & 0.0030 & 2.403 & 0.053 & 0.0837 & 0.0014 & 0.658 & 1219 & 16 & 1286 & 32 & 5 \\
\hline 14.1 & 625 & 149 & 0.24 & 179.3 & 0.000133 & 0.21 & 2.995 & 0.032 & 0.1162 & 0.0004 & 0.3332 & 0.0036 & 5.255 & 0.062 & 0.1144 & 0.0006 & 0.907 & 1854 & 17 & 1870 & 9 & 1 \\
\hline 15.1 & 268 & 117 & 0.44 & 75.0 & - & $<0.01$ & 3.073 & 0.036 & 0.1098 & 0.0006 & 0.3255 & 0.0038 & 4.932 & 0.064 & 0.1099 & 0.0006 & 0.898 & 1816 & 18 & 1798 & 10 & -1 \\
\hline 16.1 & 474 & 166 & 0.35 & 131.1 & 0.000002 & $<0.01$ & 3.107 & 0.034 & 0.1107 & 0.0005 & 0.3219 & 0.0035 & 4.913 & 0.058 & 0.1107 & 0.0005 & 0.932 & 1799 & 17 & 1811 & 8 & 1 \\
\hline 17.1 & 524 & 189 & 0.36 & 141.1 & - & $<0.01$ & 3.191 & 0.035 & 0.1103 & 0.0005 & 0.3134 & 0.0034 & 4.770 & 0.056 & 0.1104 & 0.0005 & 0.932 & 1757 & 17 & 1806 & 8 & 3 \\
\hline 18.1 & 32 & 21 & 0.65 & 5.0 & 0.000641 & 1.10 & 5.628 & 0.114 & 0.0784 & 0.0020 & 0.1765 & 0.0036 & 1.778 & 0.062 & 0.0731 & 0.0021 & 0.582 & 1048 & 22 & 1016 & 57 & -3 \\
\hline 19.1 & 181 & 90 & 0.50 & 43.5 & - & $<0.01$ & 3.566 & 0.044 & 0.0982 & 0.0008 & 0.2805 & 0.0035 & 3.797 & 0.055 & 0.0982 & 0.0008 & 0.850 & 1594 & 17 & 1590 & 14 & 0 \\
\hline 20.1 & 287 & 65 & 0.23 & 79.2 & 0.000015 & 0.02 & 3.115 & 0.036 & 0.1101 & 0.0006 & 0.3209 & 0.0037 & 4.863 & 0.062 & 0.1099 & 0.0006 & 0.900 & 1794 & 18 & 1798 & 10 & 0 \\
\hline 21.1 & 186 & 35 & 0.19 & 48.5 & 0.000036 & 0.06 & 3.286 & 0.040 & 0.1126 & 0.0008 & 0.3041 & 0.0037 & 4.702 & 0.068 & 0.1121 & 0.0008 & 0.855 & 1712 & 18 & 1834 & 14 & 7 \\
\hline 22.1 & 184 & 152 & 0.83 & 43.3 & 0.000197 & 0.31 & 3.645 & 0.045 & 0.1123 & 0.0008 & 0.2735 & 0.0034 & 4.135 & 0.069 & 0.1096 & 0.0012 & 0.748 & 1559 & 17 & 1794 & 20 & 13 \\
\hline 23.1 & 123 & 73 & 0.59 & 16.6 & 0.000062 & 0.11 & 6.363 & 0.090 & 0.0730 & 0.0011 & 0.1570 & 0.0022 & 1.561 & 0.033 & 0.0721 & 0.0012 & 0.657 & 940 & 12 & 989 & 33 & 5 \\
\hline 24.1 & 174 & 31 & 0.18 & 38.6 & 0.000031 & 0.05 & 3.879 & 0.054 & 0.0919 & 0.0008 & 0.2577 & 0.0036 & 3.251 & 0.055 & 0.0915 & 0.0008 & 0.834 & 1478 & 19 & 1457 & 18 & -1 \\
\hline 25.1 & 1192 & 214 & 0.18 & 302.5 & 0.000202 & 0.31 & 3.386 & 0.035 & 0.1147 & 0.0003 & 0.2944 & 0.0031 & 4.546 & 0.053 & 0.1120 & 0.0006 & 0.894 & 1663 & 15 & 1832 & 9 & 9 \\
\hline 26.1 & 62 & 17 & 0.28 & 17.3 & 0.000064 & 0.10 & 3.098 & 0.047 & 0.1143 & 0.0013 & 0.3225 & 0.0049 & 5.046 & 0.097 & 0.1135 & 0.0013 & 0.792 & 1802 & 24 & 1856 & 21 & 3 \\
\hline 27.1 & 204 & 63 & 0.31 & 56.2 & 0.000030 & 0.05 & 3.120 & 0.038 & 0.1101 & 0.0007 & 0.3204 & 0.0039 & 4.844 & 0.068 & 0.1097 & 0.0008 & 0.860 & 1792 & 19 & 1794 & 13 & 0 \\
\hline 28.1 & 404 & 136 & 0.34 & 94.7 & 0.000042 & 0.07 & 3.669 & 0.041 & 0.1098 & 0.0006 & 0.2724 & 0.0030 & 4.104 & 0.051 & 0.1093 & 0.0006 & 0.895 & 1553 & 15 & 1787 & 10 & 13 \\
\hline 29.1 & 229 & 50 & 0.22 & 60.7 & 0.000026 & 0.04 & 3.239 & 0.039 & 0.1110 & 0.0007 & 0.3086 & 0.0037 & 4.706 & 0.064 & 0.1106 & 0.0007 & 0.881 & 1734 & 18 & 1809 & 12 & 4 \\
\hline 30.1 & 381 & 85 & 0.22 & 106.5 & - & $<0.01$ & 3.075 & 0.034 & 0.1146 & 0.0005 & 0.3253 & 0.0036 & 5.143 & 0.063 & 0.1147 & 0.0005 & 0.920 & 1815 & 18 & 1875 & 9 & 3 \\
\hline 31.1 & 168 & 46 & 0.28 & 46.9 & 0.000098 & 0.15 & 3.081 & 0.042 & 0.1108 & 0.0009 & 0.3240 & 0.0045 & 4.890 & 0.085 & 0.1094 & 0.0012 & 0.789 & 1809 & 22 & 1790 & 19 & -1 \\
\hline
\end{tabular}




\begin{tabular}{|c|c|c|c|c|c|c|c|c|c|c|c|c|c|c|c|c|c|c|c|c|c|c|}
\hline 32.1 & 214 & 63 & 0.30 & 61.9 & - & $<0.01$ & 2.965 & 0.038 & 0.1099 & 0.0008 & 0.3377 & 0.0044 & 5.169 & 0.085 & 0.1110 & 0.0011 & 0.785 & 1876 & 21 & 1816 & 19 & -3 \\
\hline 33.1 & 508 & 122 & 0.24 & 146.4 & 0.002040 & 3.20 & 2.979 & 0.036 & 0.1343 & 0.0006 & 0.3249 & 0.0040 & 4.771 & 0.194 & 0.1065 & 0.0041 & 0.305 & 1814 & 20 & 1740 & 71 & -4 \\
\hline 34.1 & 578 & 125 & 0.22 & 174.6 & 0.000007 & 0.01 & 2.844 & 0.032 & 0.1149 & 0.0005 & 0.3516 & 0.0040 & 5.568 & 0.068 & 0.1149 & 0.0005 & 0.932 & 1942 & 19 & 1878 & 8 & -3 \\
\hline 35.1 & 53 & 25 & 0.47 & 13.8 & - & $<0.01$ & 3.279 & 0.067 & 0.1018 & 0.0016 & 0.3057 & 0.0063 & 4.387 & 0.125 & 0.1041 & 0.0021 & 0.718 & 1720 & 31 & 1698 & 37 & -1 \\
\hline 36.1 & 435 & 211 & 0.49 & 104.8 & 0.000947 & 1.49 & 3.562 & 0.043 & 0.1194 & 0.0007 & 0.2766 & 0.0034 & 4.059 & 0.103 & 0.1064 & 0.0024 & 0.481 & 1574 & 17 & 1739 & 41 & 9 \\
\hline 37.1 & 1205 & 264 & 0.22 & 183.1 & 0.000179 & 0.29 & 5.652 & 0.062 & 0.1008 & 0.0005 & 0.1764 & 0.0019 & 2.392 & 0.032 & 0.0983 & 0.0007 & 0.830 & 1047 & 11 & 1593 & 14 & 34 \\
\hline 38.1 & 135 & 31 & 0.23 & 38.0 & - & $<0.01$ & 3.050 & 0.046 & 0.1104 & 0.0011 & 0.3280 & 0.0050 & 5.005 & 0.090 & 0.1107 & 0.0011 & 0.839 & 1829 & 24 & 1811 & 18 & -1 \\
\hline 39.1 & 84 & 21 & 0.24 & 24.5 & 0.000136 & 0.21 & 2.960 & 0.052 & 0.1097 & 0.0013 & 0.3380 & 0.0059 & 5.137 & 0.109 & 0.1102 & 0.0013 & 0.822 & 1877 & 29 & 1803 & 22 & -4 \\
\hline 40.1 & 639 & 131 & 0.20 & 189.5 & 0.000039 & 0.06 & 2.900 & 0.033 & 0.1138 & 0.0005 & 0.3451 & 0.0040 & 5.435 & 0.067 & 0.1142 & 0.0005 & 0.933 & 1911 & 20 & 1868 & 8 & -2 \\
\hline 41.1 & 306 & 96 & 0.31 & 84.5 & 0.000094 & 0.15 & 3.109 & 0.040 & 0.1110 & 0.0008 & 0.3212 & 0.0041 & 4.860 & 0.076 & 0.1098 & 0.0010 & 0.822 & 1795 & 20 & 1795 & 16 & 0 \\
\hline 42.1 & 2172 & 1045 & 0.48 & 483.3 & 0.005814 & 9.16 & 3.860 & 0.041 & 0.1842 & 0.0005 & 0.2353 & 0.0029 & 3.399 & 0.381 & 0.1048 & 0.0117 & 0.112 & 1362 & 15 & 1710 & 205 & 20 \\
\hline 43.1 & 194 & 47 & 0.24 & 49.3 & 0.000098 & 0.15 & 3.373 & 0.049 & 0.1117 & 0.0010 & 0.2960 & 0.0043 & 4.503 & 0.084 & 0.1103 & 0.0013 & 0.779 & 1672 & 21 & 1805 & 21 & 7 \\
\hline 44.1 & 334 & 260 & 0.78 & 69.4 & 0.000024 & 0.04 & 4.127 & 0.054 & 0.0900 & 0.0008 & 0.2422 & 0.0032 & 2.995 & 0.050 & 0.0897 & 0.0009 & 0.791 & 1398 & 17 & 1419 & 20 & 1 \\
\hline 45.1 & 132 & 101 & 0.76 & 32.7 & 0.000163 & 0.26 & 3.473 & 0.057 & 0.1028 & 0.0015 & 0.2872 & 0.0047 & 3.982 & 0.098 & 0.1006 & 0.0018 & 0.666 & 1628 & 23 & 1635 & 34 & 0 \\
\hline 46.1 & 129 & 21 & 0.16 & 35.8 & - & $<0.01$ & 3.086 & 0.050 & 0.1093 & 0.0012 & 0.3243 & 0.0053 & 4.919 & 0.113 & 0.1100 & 0.0018 & 0.713 & 1811 & 26 & 1800 & 29 & -1 \\
\hline 47.1 & 364 & 120 & 0.33 & 81.3 & 0.000042 & 0.07 & 3.845 & 0.049 & 0.0996 & 0.0008 & 0.2599 & 0.0033 & 3.548 & 0.057 & 0.0990 & 0.0010 & 0.797 & 1489 & 17 & 1606 & 18 & 7 \\
\hline 48.1 & 81 & 74 & 0.91 & 19.8 & 0.000152 & 0.24 & 3.525 & 0.068 & 0.1020 & 0.0015 & 0.2830 & 0.0055 & 3.898 & 0.105 & 0.0999 & 0.0019 & 0.718 & 1607 & 27 & 1622 & 35 & 1 \\
\hline 49.1 & 605 & 135 & 0.22 & 175.1 & 0.000012 & 0.02 & 2.971 & 0.035 & 0.1142 & 0.0005 & 0.3366 & 0.0039 & 5.292 & 0.067 & 0.1140 & 0.0006 & 0.920 & 1870 & 19 & 1865 & 9 & 0 \\
\hline 50.1 & 211 & 74 & 0.35 & 58.5 & 0.000023 & 0.04 & 3.097 & 0.044 & 0.1091 & 0.0009 & 0.3236 & 0.0046 & 4.944 & 0.082 & 0.1108 & 0.0009 & 0.858 & 1807 & 23 & 1813 & 15 & 0 \\
\hline 51.1 & 167 & 125 & 0.75 & 22.9 & 0.000013 & 0.02 & 6.274 & 0.099 & 0.0715 & 0.0012 & 0.1594 & 0.0025 & 1.568 & 0.036 & 0.0714 & 0.0012 & 0.681 & 953 & 14 & 968 & 35 & 1 \\
\hline 52.1 & 359 & 108 & 0.30 & 85.4 & 0.000032 & 0.05 & 3.613 & 0.046 & 0.1006 & 0.0008 & 0.2767 & 0.0035 & 3.821 & 0.061 & 0.1002 & 0.0010 & 0.793 & 1575 & 18 & 1627 & 18 & 3 \\
\hline 53.1 & 173 & 86 & 0.50 & 38.8 & 0.000095 & 0.15 & 3.836 & 0.064 & 0.0936 & 0.0013 & 0.2607 & 0.0043 & 3.358 & 0.072 & 0.0934 & 0.0013 & 0.776 & 1493 & 24 & 1497 & 26 & 0 \\
\hline 54.1 & 256 & 64 & 0.25 & 69.7 & 0.000070 & 0.11 & 3.161 & 0.045 & 0.1117 & 0.0010 & 0.3160 & 0.0045 & 4.826 & 0.087 & 0.1108 & 0.0012 & 0.793 & 1770 & 22 & 1812 & 20 & 2 \\
\hline 55.1 & 837 & 287 & 0.34 & 221.5 & 0.000038 & 0.06 & 3.244 & 0.037 & 0.1094 & 0.0006 & 0.3081 & 0.0035 & 4.627 & 0.059 & 0.1089 & 0.0006 & 0.895 & 1731 & 17 & 1782 & 10 & 3 \\
\hline 56.1 & 68 & 13 & 0.20 & 15.8 & - & $<0.01$ & 3.664 & 0.089 & 0.1112 & 0.0017 & 0.2730 & 0.0066 & 4.194 & 0.121 & 0.1114 & 0.0017 & 0.840 & 1556 & 34 & 1823 & 28 & 15 \\
\hline 58.1 & 164 & 44 & 0.27 & 45.5 & 0.000085 & 0.13 & 3.093 & 0.049 & 0.1099 & 0.0011 & 0.3237 & 0.0052 & 4.947 & 0.094 & 0.1108 & 0.0011 & 0.839 & 1808 & 26 & 1813 & 19 & 0 \\
\hline 59.1 & 278 & 90 & 0.32 & 54.7 & - & $<0.01$ & 4.373 & 0.063 & 0.0874 & 0.0010 & 0.2289 & 0.0033 & 2.783 & 0.064 & 0.0882 & 0.0016 & 0.634 & 1329 & 18 & 1387 & 34 & 4 \\
\hline 60.1 & 19 & 19 & 0.99 & 4.5 & 0.000058 & 0.09 & 3.567 & 0.131 & 0.1048 & 0.0035 & 0.2787 & 0.0102 & 3.840 & 0.196 & 0.0999 & 0.0035 & 0.720 & 1585 & 61 & 1623 & 66 & 2 \\
\hline
\end{tabular}

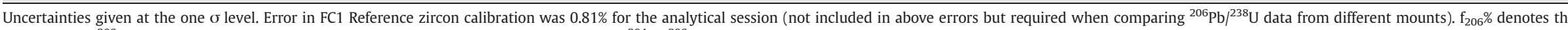
percentage of ${ }^{206} \mathrm{~Pb}$ that is common $\mathrm{Pb}$. Correction for common $\mathrm{Pb}$ made using the measured ${ }^{204} \mathrm{~Pb} /{ }^{206} \mathrm{~Pb}$ ratio. For \% Disc, $0 \%$ denotes a concordant analysis. $\mathrm{Pb}$ is the proportion of $\mathrm{Pb}$ that is radiogenic. 
Table 3

Summary of SHRIMP U-Pb zircon results for Raneälven river sand

\begin{tabular}{|c|c|c|c|c|c|c|c|c|c|c|c|c|c|c|c|c|c|c|c|c|c|c|}
\hline \multirow[b]{2}{*}{$\begin{array}{l}\text { Grain. } \\
\text { spot }\end{array}$} & \multirow[b]{2}{*}{$\begin{array}{l}\mathrm{U} \\
(\mathrm{ppm})\end{array}$} & \multirow[b]{2}{*}{$\begin{array}{l}\text { Th } \\
\text { (ppm) }\end{array}$} & \multirow[b]{2}{*}{$\mathrm{Th} / \mathrm{U}$} & \multirow[b]{2}{*}{$\begin{array}{l}\mathrm{Pb}^{*} \\
(\mathrm{ppm})\end{array}$} & \multirow[b]{2}{*}{${ }^{204} \mathrm{~Pb} /{ }^{206} \mathrm{~Pb}$} & \multirow[b]{2}{*}{$\mathrm{F}_{206} \%$} & \multicolumn{4}{|l|}{ Total ratios } & \multicolumn{6}{|c|}{ Radiogenic ratios } & \multirow[b]{2}{*}{$r$} & \multicolumn{4}{|l|}{ Age (Ma) } & \multirow[b]{2}{*}{ \% Disc } \\
\hline & & & & & & & ${ }^{238} \mathrm{U} /{ }^{206} \mathrm{~Pb}$ & \pm & ${ }^{207} \mathrm{~Pb} /{ }^{206} \mathrm{~Pb}$ & \pm & ${ }^{206} \mathrm{~Pb} /{ }^{238} \mathrm{U}$ & \pm & ${ }^{207} \mathrm{~Pb} /{ }^{235} \mathrm{U}$ & \pm & ${ }^{207} \mathrm{~Pb} /{ }^{206} \mathrm{~Pb}$ & \pm & & ${ }^{206} \mathrm{~Pb} /{ }^{238} \mathrm{U}$ & \pm & ${ }^{207} \mathrm{~Pb} /{ }^{206} \mathrm{~Pb}$ & \pm & \\
\hline 1.1 & 192 & 53 & 0.27 & 51.8 & 0.000072 & 0.11 & 3.181 & 0.047 & 0.1104 & 0.0010 & 0.3140 & 0.0047 & 4.738 & 0.087 & 0.1094 & 0.0012 & 0.809 & 1760 & 23 & 1790 & 20 & 2 \\
\hline 2.1 & 298 & 149 & 0.50 & 82.5 & 0.000022 & 0.03 & 3.101 & 0.041 & 0.1149 & 0.0008 & 0.3223 & 0.0043 & 5.093 & 0.078 & 0.1146 & 0.0009 & 0.871 & 1801 & 21 & 1874 & 14 & 4 \\
\hline 3.1 & 615 & 183 & 0.30 & 141.1 & 0.000203 & 0.31 & 3.742 & 0.045 & 0.1148 & 0.0007 & 0.2664 & 0.0032 & 4.115 & 0.061 & 0.1120 & 0.0010 & 0.802 & 1523 & 16 & 1832 & 16 & 17 \\
\hline 4.1 & 88 & 37 & 0.42 & 24.7 & - & $<0.01$ & 3.045 & 0.059 & 0.1171 & 0.0016 & 0.3292 & 0.0064 & 5.412 & 0.138 & 0.1192 & 0.0020 & 0.759 & 1834 & 31 & 1945 & 30 & 6 \\
\hline 5.1 & 122 & 61 & 0.50 & 35.1 & - & $<0.01$ & 2.980 & 0.051 & 0.1123 & 0.0013 & 0.3368 & 0.0057 & 5.343 & 0.110 & 0.1151 & 0.0013 & 0.824 & 1871 & 29 & 1881 & 21 & 1 \\
\hline 6.1 & 220 & 101 & 0.46 & 57.4 & 0.000034 & 0.05 & 3.293 & 0.048 & 0.1171 & 0.0011 & 0.3035 & 0.0044 & 4.881 & 0.086 & 0.1166 & 0.0012 & 0.826 & 1709 & 22 & 1905 & 18 & 10 \\
\hline 7.1 & 335 & 155 & 0.46 & 93.8 & 0.000018 & 0.03 & 3.072 & 0.041 & 0.1154 & 0.0008 & 0.3255 & 0.0043 & 5.167 & 0.078 & 0.1151 & 0.0008 & 0.878 & 1816 & 21 & 1882 & 13 & 3 \\
\hline 8.1 & 220 & 70 & 0.32 & 62.1 & 0.000109 & 0.17 & 3.045 & 0.044 & 0.1174 & 0.0010 & 0.3278 & 0.0048 & 5.239 & 0.106 & 0.1159 & 0.0016 & 0.724 & 1828 & 23 & 1894 & 25 & 3 \\
\hline 9.1 & 520 & 470 & 0.90 & 123.3 & 0.000009 & 0.01 & 3.620 & 0.044 & 0.1028 & 0.0007 & 0.2762 & 0.0034 & 3.909 & 0.055 & 0.1026 & 0.0007 & 0.862 & 1572 & 17 & 1672 & 13 & 6 \\
\hline 10.1 & 158 & 64 & 0.40 & 41.9 & - & $<0.01$ & 3.238 & 0.053 & 0.1116 & 0.0016 & 0.3089 & 0.0051 & 4.769 & 0.105 & 0.1120 & 0.0017 & 0.742 & 1736 & 25 & 1831 & 27 & 5 \\
\hline 11.1 & 225 & 219 & 0.97 & 64.0 & 0.000044 & 0.07 & 3.019 & 0.050 & 0.1164 & 0.0010 & 0.3310 & 0.0055 & 5.286 & 0.114 & 0.1158 & 0.0016 & 0.763 & 1843 & 26 & 1893 & 25 & 3 \\
\hline 12.1 & 142 & 84 & 0.59 & 40.3 & 0.000049 & 0.08 & 3.034 & 0.050 & 0.1158 & 0.0013 & 0.3294 & 0.0055 & 5.231 & 0.115 & 0.1152 & 0.0016 & 0.758 & 1835 & 27 & 1883 & 26 & 3 \\
\hline 13.1 & 129 & 107 & 0.83 & 37.6 & 0.000094 & 0.15 & 2.938 & 0.059 & 0.1138 & 0.0014 & 0.3413 & 0.0069 & 5.466 & 0.128 & 0.1161 & 0.0014 & 0.860 & 1893 & 37 & 1898 & 21 & 0 \\
\hline 14.1 & 80 & 34 & 0.43 & 22.7 & - & $<0.01$ & 3.027 & 0.063 & 0.1163 & 0.0018 & 0.3316 & 0.0070 & 5.470 & 0.184 & 0.1196 & 0.0031 & 0.624 & 1846 & 34 & 1951 & 47 & 5 \\
\hline 15.1 & 115 & 60 & 0.52 & 35.5 & - & $<0.01$ & 2.794 & 0.044 & 0.1141 & 0.0012 & 0.3580 & 0.0056 & 5.654 & 0.109 & 0.1145 & 0.0013 & 0.818 & 1973 & 27 & 1873 & 20 & -5 \\
\hline 16.1 & 156 & 84 & 0.54 & 46.8 & - & $<0.01$ & 2.852 & 0.043 & 0.1112 & 0.0011 & 0.3513 & 0.0053 & 5.469 & 0.098 & 0.1129 & 0.0011 & 0.845 & 1941 & 27 & 1847 & 17 & -5 \\
\hline 17.1 & 131 & 44 & 0.34 & 39.6 & 0.000285 & 0.44 & 2.830 & 0.044 & 0.1154 & 0.0012 & 0.3522 & 0.0054 & 5.468 & 0.104 & 0.1126 & 0.0012 & 0.812 & 1945 & 27 & 1842 & 20 & -6 \\
\hline 18.1 & 174 & 69 & 0.40 & 49.8 & 0.000009 & 0.01 & 2.996 & 0.050 & 0.1160 & 0.0011 & 0.3337 & 0.0056 & 5.333 & 0.104 & 0.1159 & 0.0011 & 0.862 & 1856 & 27 & 1894 & 18 & 2 \\
\hline 19.1 & 158 & 200 & 1.26 & 50.4 & - & $<0.01$ & 2.700 & 0.040 & 0.1162 & 0.0010 & 0.3709 & 0.0055 & 6.001 & 0.104 & 0.1173 & 0.0010 & 0.859 & 2034 & 31 & 1916 & 16 & -6 \\
\hline 20.1 & 158 & 77 & 0.49 & 51.8 & - & $<0.01$ & 2.616 & 0.041 & 0.1153 & 0.0011 & 0.3824 & 0.0060 & 6.092 & 0.112 & 0.1155 & 0.0011 & 0.850 & 2088 & 28 & 1888 & 17 & -11 \\
\hline 21.1 & 294 & 487 & 1.66 & 71.6 & - & $<0.01$ & 3.527 & 0.052 & 0.0821 & 0.0008 & 0.2861 & 0.0043 & 3.536 & 0.071 & 0.0896 & 0.0012 & 0.745 & 1622 & 32 & 1418 & 25 & -14 \\
\hline 22.1 & 65 & 89 & 1.36 & 10.4 & - & $<0.01$ & 5.415 & 0.118 & 0.0756 & 0.0018 & 0.1858 & 0.0041 & 2.069 & 0.101 & 0.0808 & 0.0035 & 0.449 & 1099 & 22 & 1216 & 86 & 10 \\
\hline 23.1 & 101 & 13 & 0.13 & 31.5 & 0.000050 & 0.08 & 2.753 & 0.049 & 0.1157 & 0.0014 & 0.3640 & 0.0065 & 5.888 & 0.127 & 0.1173 & 0.0014 & 0.823 & 2001 & 31 & 1916 & 22 & -4 \\
\hline 24.1 & 85 & 57 & 0.67 & 24.5 & 0.000156 & 0.25 & 2.982 & 0.059 & 0.1078 & 0.0016 & 0.3357 & 0.0066 & 5.028 & 0.123 & 0.1086 & 0.0016 & 0.810 & 1866 & 35 & 1777 & 26 & -5 \\
\hline 25.1 & 345 & 262 & 0.76 & 100.4 & 0.000080 & 0.12 & 2.953 & 0.039 & 0.1159 & 0.0008 & 0.3382 & 0.0045 & 5.355 & 0.087 & 0.1148 & 0.0011 & 0.817 & 1878 & 22 & 1877 & 17 & 0 \\
\hline 26.1 & 355 & 123 & 0.35 & 102.2 & - & $<0.01$ & 2.985 & 0.040 & 0.1139 & 0.0009 & 0.3351 & 0.0045 & 5.274 & 0.082 & 0.1142 & 0.0009 & 0.862 & 1863 & 22 & 1867 & 14 & 0 \\
\hline 27.1 & 268 & 96 & 0.36 & 75.0 & 0.000021 & 0.03 & 3.071 & 0.043 & 0.1114 & 0.0009 & 0.3255 & 0.0046 & 4.987 & 0.083 & 0.1111 & 0.0010 & 0.852 & 1817 & 22 & 1817 & 16 & 0 \\
\hline 28.1 & 170 & 87 & 0.51 & 50.4 & - & $<0.01$ & 2.901 & 0.046 & 0.1165 & 0.0013 & 0.3449 & 0.0055 & 5.563 & 0.109 & 0.1170 & 0.0013 & 0.817 & 1910 & 26 & 1911 & 20 & 0 \\
\hline 29.1 & 171 & 119 & 0.70 & 46.1 & 0.000319 & 0.49 & 3.180 & 0.051 & 0.1159 & 0.0013 & 0.3129 & 0.0050 & 4.815 & 0.102 & 0.1116 & 0.0016 & 0.751 & 1755 & 24 & 1826 & 25 & 4 \\
\hline
\end{tabular}




\begin{tabular}{|c|c|c|c|c|c|c|c|c|c|c|c|c|c|c|c|c|c|c|c|c|c|c|}
\hline 30.1 & 139 & 36 & 0.26 & 42.7 & - & $<0.01$ & 2.805 & 0.050 & 0.1123 & 0.0014 & 0.3567 & 0.0064 & 5.546 & 0.130 & 0.1128 & 0.0017 & 0.767 & 1967 & 30 & 1844 & 27 & -7 \\
\hline 31.1 & 172 & 59 & 0.35 & 25.8 & 0.000644 & 1.02 & 5.711 & 0.102 & 0.1111 & 0.0018 & 0.1692 & 0.0030 & 1.938 & 0.098 & 0.0831 & 0.0039 & 0.353 & 1008 & 19 & 1271 & 93 & 21 \\
\hline 32.1 & 447 & 234 & 0.52 & 120.7 & 0.000019 & 0.03 & 3.180 & 0.044 & 0.1080 & 0.0009 & 0.3144 & 0.0043 & 4.670 & 0.077 & 0.1078 & 0.0010 & 0.839 & 1762 & 21 & 1762 & 16 & 0 \\
\hline 33.1 & 275 & 81 & 0.30 & 81.5 & 0.000043 & 0.07 & 2.903 & 0.044 & 0.1099 & 0.0010 & 0.3460 & 0.0053 & 5.413 & 0.098 & 0.1135 & 0.0011 & 0.842 & 1915 & 26 & 1856 & 18 & -3 \\
\hline 34.1 & 184 & 153 & 0.83 & 49.9 & 0.000084 & 0.13 & 3.169 & 0.055 & 0.1095 & 0.0014 & 0.3151 & 0.0055 & 4.707 & 0.111 & 0.1083 & 0.0017 & 0.735 & 1766 & 27 & 1771 & 29 & 0 \\
\hline 35.1 & 216 & 129 & 0.60 & 66.2 & - & $<0.01$ & 2.811 & 0.046 & 0.1130 & 0.0014 & 0.3562 & 0.0058 & 5.602 & 0.118 & 0.1141 & 0.0015 & 0.774 & 1964 & 28 & 1865 & 24 & -5 \\
\hline 36.1 & 223 & 119 & 0.54 & 68.3 & - & $<0.01$ & 2.803 & 0.050 & 0.1165 & 0.0012 & 0.3574 & 0.0063 & 5.801 & 0.119 & 0.1177 & 0.0012 & 0.865 & 1970 & 32 & 1922 & 18 & -2 \\
\hline 37.1 & 106 & 53 & 0.49 & 30.7 & 0.000131 & 0.21 & 2.976 & 0.059 & 0.1096 & 0.0016 & 0.3356 & 0.0067 & 5.028 & 0.125 & 0.1087 & 0.0016 & 0.803 & 1866 & 35 & 1777 & 27 & -5 \\
\hline 38.1 & 102 & 38 & 0.37 & 32.2 & 0.000021 & 0.03 & 2.722 & 0.055 & 0.1148 & 0.0016 & 0.3682 & 0.0075 & 5.921 & 0.147 & 0.1166 & 0.0017 & 0.818 & 2021 & 37 & 1905 & 26 & -6 \\
\hline 39.1 & 89 & 80 & 0.90 & 25.8 & - & $<0.01$ & 2.962 & 0.066 & 0.1150 & 0.0018 & 0.3386 & 0.0075 & 5.487 & 0.160 & 0.1175 & 0.0022 & 0.759 & 1880 & 36 & 1919 & 34 & 2 \\
\hline 40.1 & 357 & 7864 & 22.0 & 88.3 & 0.001648 & 2.55 & 3.478 & 0.048 & 0.1357 & 0.0016 & 0.2802 & 0.0041 & 4.383 & 0.200 & 0.1135 & 0.0049 & 0.320 & 1592 & 21 & 1855 & 78 & 14 \\
\hline 41.1 & 235 & 111 & 0.47 & 65.3 & 0.000066 & 0.10 & 3.093 & 0.048 & 0.1159 & 0.0011 & 0.3230 & 0.0050 & 5.121 & 0.096 & 0.1150 & 0.0012 & 0.820 & 1804 & 24 & 1880 & 19 & 4 \\
\hline 42.1 & 24 & 14 & 0.58 & 4.7 & - & $<0.01$ & 4.291 & 0.168 & 0.0864 & 0.0037 & 0.2348 & 0.0098 & 3.009 & 0.442 & 0.0929 & 0.0131 & 0.285 & 1360 & 51 & 1487 & 266 & 9 \\
\hline 43.1 & 278 & 147 & 0.53 & 80.8 & 0.000037 & 0.06 & 2.953 & 0.043 & 0.1155 & 0.0010 & 0.3385 & 0.0049 & 5.367 & 0.097 & 0.1150 & 0.0012 & 0.804 & 1879 & 24 & 1880 & 19 & 0 \\
\hline 44.1 & 369 & 240 & 0.65 & 108.1 & 0.000005 & 0.01 & 2.932 & 0.039 & 0.1161 & 0.0008 & 0.3410 & 0.0046 & 5.457 & 0.083 & 0.1161 & 0.0008 & 0.878 & 1892 & 22 & 1896 & 13 & 0 \\
\hline 45.1 & 104 & 88 & 0.84 & 31.5 & 0.000160 & 0.25 & 2.839 & 0.060 & 0.1124 & 0.0016 & 0.3546 & 0.0075 & 5.759 & 0.149 & 0.1178 & 0.0018 & 0.815 & 1957 & 40 & 1923 & 27 & -2 \\
\hline 46.1 & 235 & 119 & 0.51 & 69.4 & 0.000154 & 0.24 & 2.904 & 0.043 & 0.1157 & 0.0010 & 0.3454 & 0.0051 & 5.622 & 0.098 & 0.1181 & 0.0011 & 0.855 & 1913 & 26 & 1927 & 16 & 1 \\
\hline 47.1 & 263 & 87 & 0.33 & 77.1 & 0.000070 & 0.11 & 2.930 & 0.042 & 0.1138 & 0.0010 & 0.3410 & 0.0049 & 5.307 & 0.089 & 0.1129 & 0.0010 & 0.848 & 1891 & 23 & 1846 & 16 & -2 \\
\hline 48.1 & 476 & 104 & 0.22 & 138.4 & 0.024495 & 28.74 & 2.958 & 0.382 & 0.5565 & 0.0201 & 0.2409 & 0.0362 & 10.498 & 3.111 & 0.3160 & 0.0807 & 0.507 & 1392 & 188 & 3551 & 393 & 61 \\
\hline 49.1 & 75 & 36 & 0.48 & 21.0 & - & $<0.01$ & 3.081 & 0.069 & 0.1107 & 0.0019 & 0.3251 & 0.0073 & 5.024 & 0.151 & 0.1121 & 0.0022 & 0.746 & 1815 & 35 & 1834 & 36 & 1 \\
\hline 50.1 & 157 & 63 & 0.40 & 45.9 & - & $<0.01$ & 2.944 & 0.049 & 0.1094 & 0.0013 & 0.3400 & 0.0057 & 5.166 & 0.111 & 0.1102 & 0.0015 & 0.786 & 1887 & 28 & 1802 & 24 & -5 \\
\hline 51.1 & 170 & 58 & 0.34 & 49.9 & 0.000054 & 0.08 & 2.932 & 0.048 & 0.1141 & 0.0013 & 0.3407 & 0.0055 & 5.325 & 0.109 & 0.1133 & 0.0014 & 0.795 & 1890 & 27 & 1854 & 22 & -2 \\
\hline 52.1 & 300 & 162 & 0.54 & 90.7 & 0.000063 & 0.10 & 2.845 & 0.040 & 0.1156 & 0.0009 & 0.3511 & 0.0049 & 5.557 & 0.093 & 0.1148 & 0.0011 & 0.831 & 1940 & 23 & 1876 & 17 & -3 \\
\hline 53.1 & 171 & 76 & 0.45 & 54.8 & - & $<0.01$ & 2.688 & 0.051 & 0.1166 & 0.0012 & 0.3726 & 0.0071 & 6.058 & 0.148 & 0.1179 & 0.0018 & 0.774 & 2041 & 33 & 1925 & 28 & -6 \\
\hline 54.1 & 331 & 235 & 0.71 & 93.9 & 0.000044 & 0.07 & 3.029 & 0.052 & 0.1155 & 0.0013 & 0.3299 & 0.0057 & 5.227 & 0.108 & 0.1149 & 0.0013 & 0.833 & 1838 & 27 & 1879 & 21 & 2 \\
\hline 55.1 & 172 & 76 & 0.44 & 51.8 & 0.000160 & 0.25 & 2.847 & 0.047 & 0.1153 & 0.0013 & 0.3503 & 0.0057 & 5.463 & 0.119 & 0.1131 & 0.0016 & 0.751 & 1936 & 27 & 1850 & 26 & -5 \\
\hline 56.1 & 349 & 248 & 0.71 & 97.8 & - & $<0.01$ & 3.064 & 0.046 & 0.1167 & 0.0009 & 0.3265 & 0.0049 & 5.270 & 0.090 & 0.1171 & 0.0009 & 0.882 & 1821 & 24 & 1912 & 14 & 5 \\
\hline 57.1 & 798 & 500 & 0.63 & 264.9 & 0.000572 & 0.88 & 2.588 & 0.031 & 0.1226 & 0.0006 & 0.3836 & 0.0046 & 6.175 & 0.088 & 0.1168 & 0.0009 & 0.831 & 2093 & 23 & 1907 & 14 & -10 \\
\hline 58.1 & 151 & 56 & 0.37 & 45.4 & - & $<0.01$ & 2.852 & 0.049 & 0.1158 & 0.0013 & 0.3518 & 0.0060 & 5.749 & 0.118 & 0.1185 & 0.0013 & 0.832 & 1943 & 30 & 1934 & 20 & 0 \\
\hline 59.1 & 181 & 45 & 0.25 & 79.9 & 0.000086 & 0.13 & 1.944 & 0.037 & 0.1146 & 0.0011 & 0.5164 & 0.0098 & 8.383 & 0.180 & 0.1177 & 0.0012 & 0.882 & 2684 & 43 & 1922 & 18 & -40 \\
\hline 60.1 & 263 & 136 & 0.51 & 78.9 & - & $<0.01$ & 2.866 & 0.046 & 0.1149 & 0.0010 & 0.3491 & 0.0056 & 5.551 & 0.102 & 0.1153 & 0.0010 & 0.874 & 1930 & 27 & 1885 & 16 & -2 \\
\hline
\end{tabular}

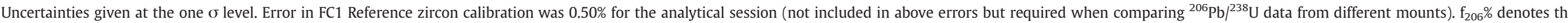
percentage of ${ }^{206} \mathrm{~Pb}$ that is common $\mathrm{Pb}$. Correction for common $\mathrm{Pb}$ made using the measured ${ }^{204} \mathrm{~Pb} /{ }^{206} \mathrm{~Pb}$ ratio. For \% Disc, $0 \%$ denotes a concordant analysis. Pb* is the proportion of $\mathrm{Pb}$ that is radiogenic. 
Table 4

Summary of SHRIMP U-Pb zircon results for Lainioälven river sand

\begin{tabular}{|c|c|c|c|c|c|c|c|c|c|c|c|c|c|c|c|c|c|c|c|c|c|c|}
\hline \multirow[b]{2}{*}{$\begin{array}{l}\text { Grain. } \\
\text { spot }\end{array}$} & \multirow[b]{2}{*}{$\begin{array}{l}\mathrm{U} \\
(\mathrm{ppm})\end{array}$} & \multirow[b]{2}{*}{$\begin{array}{l}\text { Th } \\
(\mathrm{ppm})\end{array}$} & \multirow[b]{2}{*}{$\mathrm{Th} / \mathrm{U}$} & \multirow[b]{2}{*}{$\begin{array}{l}\mathrm{Pb}^{*} \\
(\mathrm{ppm})\end{array}$} & \multirow[b]{2}{*}{${ }^{204} \mathrm{~Pb} /{ }^{206} \mathrm{~Pb}$} & \multirow[b]{2}{*}{$f_{206} \%$} & \multicolumn{4}{|l|}{ Total ratios } & \multicolumn{6}{|c|}{ Radiogenic ratios } & \multirow[b]{2}{*}{$\mathrm{r}$} & \multicolumn{4}{|l|}{ Age (Ma) } & \multirow[b]{2}{*}{$\%$ Disc } \\
\hline & & & & & & & ${ }^{238} \mathrm{U} /{ }^{206} \mathrm{~Pb}$ & \pm & ${ }^{207} \mathrm{~Pb} /{ }^{206} \mathrm{~Pb}$ & \pm & ${ }^{206} \mathrm{~Pb} /{ }^{238} \mathrm{U}$ & \pm & ${ }^{207} \mathrm{~Pb} /{ }^{235} \mathrm{U}$ & \pm & ${ }^{207} \mathrm{~Pb} /{ }^{206} \mathrm{~Pb}$ & \pm & & ${ }^{206} \mathrm{~Pb} /{ }^{238} \mathrm{U}$ & \pm & ${ }^{207} \mathrm{~Pb} /{ }^{206} \mathrm{~Pb}$ & \pm & \\
\hline 1.1 & 63 & 60 & 0.96 & 17.1 & 0.000081 & 0.13 & 3.154 & 0.062 & 0.1150 & 0.0020 & 0.3166 & 0.0062 & 4.973 & 0.142 & 0.1139 & 0.0024 & 0.686 & 1773 & 30 & 1863 & 37 & 5 \\
\hline 2.1 & 145 & 92 & 0.64 & 38.7 & 0.000086 & 0.14 & 3.210 & 0.047 & 0.1084 & 0.0012 & 0.3111 & 0.0046 & 4.599 & 0.092 & 0.1072 & 0.0014 & 0.735 & 1746 & 22 & 1752 & 25 & 0 \\
\hline 3.1 & 45 & 79 & 1.77 & 13.1 & 0.000325 & 0.51 & 2.955 & 0.067 & 0.1146 & 0.0022 & 0.3417 & 0.0077 & 5.769 & 0.171 & 0.1224 & 0.0023 & 0.764 & 1895 & 49 & 1992 & 34 & 5 \\
\hline 4.1 & 119 & 129 & 1.09 & 31.5 & 0.000079 & 0.12 & 3.232 & 0.055 & 0.1125 & 0.0013 & 0.3090 & 0.0052 & 4.749 & 0.104 & 0.1115 & 0.0016 & 0.770 & 1736 & 26 & 1823 & 25 & 5 \\
\hline 5.1 & 30 & 39 & 1.31 & 8.1 & 0.000166 & 0.26 & 3.140 & 0.133 & 0.1140 & 0.0029 & 0.3177 & 0.0135 & 4.895 & 0.323 & 0.1118 & 0.0056 & 0.646 & 1778 & 66 & 1828 & 91 & 3 \\
\hline 6.1 & 116 & 101 & 0.87 & 48.4 & 0.000126 & 0.17 & 2.056 & 0.048 & 0.1924 & 0.0015 & 0.4856 & 0.0112 & 12.777 & 0.318 & 0.1908 & 0.0017 & 0.930 & 2552 & 49 & 2749 & 15 & 7 \\
\hline 7.1 & 134 & 94 & 0.70 & 36.9 & 0.000049 & 0.08 & 3.112 & 0.050 & 0.1107 & 0.0011 & 0.3211 & 0.0052 & 4.870 & 0.105 & 0.1100 & 0.0016 & 0.748 & 1795 & 25 & 1799 & 26 & 0 \\
\hline 8.1 & 1083 & 168 & 0.16 & 179.4 & 0.000245 & 0.36 & 5.183 & 0.057 & 0.1391 & 0.0013 & 0.1922 & 0.0021 & 3.601 & 0.056 & 0.1359 & 0.0015 & 0.711 & 1133 & 12 & 2175 & 19 & 48 \\
\hline 9.1 & 313 & 163 & 0.52 & 87.6 & 0.000049 & 0.08 & 3.072 & 0.040 & 0.1142 & 0.0008 & 0.3252 & 0.0043 & 5.091 & 0.078 & 0.1135 & 0.0009 & 0.859 & 1815 & 21 & 1857 & 14 & 2 \\
\hline 10.1 & 481 & 207 & 0.43 & 116.7 & 0.000025 & 0.04 & 3.537 & 0.042 & 0.1121 & 0.0007 & 0.2826 & 0.0034 & 4.356 & 0.060 & 0.1118 & 0.0008 & 0.871 & 1604 & 17 & 1829 & 12 & 12 \\
\hline 11.1 & 45 & 52 & 1.16 & 11.9 & 0.000058 & 0.09 & 3.241 & 0.074 & 0.1113 & 0.0024 & 0.3083 & 0.0070 & 4.698 & 0.150 & 0.1105 & 0.0025 & 0.713 & 1732 & 35 & 1808 & 41 & 4 \\
\hline 12.1 & 28 & 32 & 1.13 & 8.0 & 0.000305 & 0.47 & 3.047 & 0.089 & 0.1138 & 0.0026 & 0.3267 & 0.0095 & 4.940 & 0.200 & 0.1097 & 0.0031 & 0.719 & 1822 & 46 & 1794 & 51 & -2 \\
\hline 13.1 & 50 & 67 & 1.34 & 13.5 & 0.000139 & 0.22 & 3.157 & 0.064 & 0.1105 & 0.0019 & 0.3161 & 0.0065 & 4.733 & 0.186 & 0.1086 & 0.0036 & 0.524 & 1771 & 32 & 1776 & 61 & 0 \\
\hline 14.1 & 63 & 44 & 0.70 & 17.5 & 0.000117 & 0.18 & 3.080 & 0.067 & 0.1118 & 0.0019 & 0.3241 & 0.0071 & 4.924 & 0.168 & 0.1102 & 0.0029 & 0.640 & 1810 & 35 & 1803 & 48 & 0 \\
\hline 15.1 & 89 & 76 & 0.85 & 24.5 & 0.000057 & 0.09 & 3.139 & 0.051 & 0.1087 & 0.0014 & 0.3193 & 0.0052 & 4.871 & 0.101 & 0.1106 & 0.0014 & 0.787 & 1786 & 29 & 1810 & 23 & 1 \\
\hline 16.1 & 25 & 20 & 0.81 & 6.8 & - & $<0.01$ & 3.137 & 0.083 & 0.1119 & 0.0028 & 0.3205 & 0.0086 & 5.153 & 0.215 & 0.1166 & 0.0037 & 0.642 & 1792 & 42 & 1905 & 57 & 6 \\
\hline 17.1 & 44 & 38 & 0.86 & 12.1 & 0.000058 & 0.09 & 3.120 & 0.066 & 0.1125 & 0.0021 & 0.3202 & 0.0068 & 4.932 & 0.157 & 0.1117 & 0.0027 & 0.665 & 1791 & 33 & 1828 & 43 & 2 \\
\hline 18.1 & 144 & 116 & 0.81 & 38.9 & 0.000022 & 0.03 & 3.170 & 0.047 & 0.1096 & 0.0012 & 0.3154 & 0.0047 & 4.753 & 0.091 & 0.1093 & 0.0013 & 0.781 & 1767 & 23 & 1788 & 22 & 1 \\
\hline 19.1 & 117 & 131 & 1.12 & 32.7 & 0.000146 & 0.22 & 3.065 & 0.048 & 0.1165 & 0.0013 & 0.3255 & 0.0051 & 5.139 & 0.115 & 0.1145 & 0.0018 & 0.696 & 1817 & 25 & 1872 & 29 & 3 \\
\hline 20.1 & 83 & 75 & 0.91 & 23.2 & 0.000100 & 0.16 & 3.063 & 0.053 & 0.1113 & 0.0015 & 0.3268 & 0.0057 & 5.046 & 0.112 & 0.1120 & 0.0015 & 0.782 & 1823 & 31 & 1832 & 25 & 1 \\
\hline 21.1 & 53 & 41 & 0.77 & 22.6 & 0.000092 & 0.12 & 2.007 & 0.045 & 0.1922 & 0.0023 & 0.4978 & 0.0111 & 13.117 & 0.334 & 0.1911 & 0.0024 & 0.872 & 2604 & 48 & 2752 & 20 & 5 \\
\hline 22.1 & 36 & 43 & 1.21 & 10.1 & 0.000095 & 0.15 & 3.064 & 0.075 & 0.1139 & 0.0025 & 0.3259 & 0.0081 & 5.062 & 0.239 & 0.1126 & 0.0045 & 0.528 & 1819 & 39 & 1842 & 73 & 1 \\
\hline 23.1 & 239 & 16 & 0.07 & 112.8 & 0.000036 & 0.05 & 1.818 & 0.024 & 0.1946 & 0.0011 & 0.5496 & 0.0073 & 14.712 & 0.215 & 0.1941 & 0.0011 & 0.915 & 2824 & 31 & 2778 & 10 & -2 \\
\hline 24.1 & 1728 & 9 & 0.01 & 525.3 & 0.000148 & 0.20 & 2.825 & 0.031 & 0.1796 & 0.0005 & 0.3532 & 0.0038 & 8.660 & 0.098 & 0.1778 & 0.0006 & 0.957 & 1950 & 18 & 2632 & 5 & 26 \\
\hline 25.1 & 54 & 75 & 1.40 & 21.8 & 0.000173 & 0.24 & 2.111 & 0.042 & 0.1859 & 0.0022 & 0.4727 & 0.0095 & 11.979 & 0.284 & 0.1838 & 0.0023 & 0.846 & 2495 & 41 & 2688 & 21 & 7 \\
\hline 26.1 & 105 & 86 & 0.82 & 29.4 & 0.000128 & 0.20 & 3.063 & 0.049 & 0.1114 & 0.0014 & 0.3258 & 0.0053 & 4.925 & 0.140 & 0.1096 & 0.0026 & 0.574 & 1818 & 26 & 1793 & 42 & -1 \\
\hline 27.1 & 604 & 216 & 0.36 & 241.5 & - & $<0.01$ & 2.150 & 0.028 & 0.1840 & 0.0010 & 0.4651 & 0.0061 & 11.807 & 0.166 & 0.1841 & 0.0010 & 0.926 & 2462 & 27 & 2690 & 9 & 8 \\
\hline 28.1 & 61 & 98 & 1.61 & 14.9 & 0.000704 & 1.11 & 3.493 & 0.071 & 0.1134 & 0.0020 & 0.2831 & 0.0059 & 4.051 & 0.180 & 0.1038 & 0.0041 & 0.465 & 1607 & 29 & 1693 & 73 & 5 \\
\hline 29.1 & 183 & 372 & 2.03 & 31.7 & 0.000398 & 0.62 & 4.969 & 0.083 & 0.1123 & 0.0016 & 0.2000 & 0.0034 & 2.947 & 0.085 & 0.1069 & 0.0025 & 0.580 & 1175 & 18 & 1747 & 43 & 33 \\
\hline 30.1 & 73 & 82 & 1.11 & 20.4 & - & $<0.01$ & 3.087 & 0.059 & 0.1149 & 0.0018 & 0.3239 & 0.0061 & 5.134 & 0.125 & 0.1149 & 0.0018 & 0.779 & 1809 & 30 & 1879 & 28 & 4 \\
\hline
\end{tabular}




\begin{tabular}{|c|c|c|c|c|c|c|c|c|c|c|c|c|c|c|c|c|c|c|c|c|c|c|}
\hline 31.1 & 31 & 22 & 0.69 & 13.1 & - & $<0.01$ & 2.059 & 0.054 & 0.1797 & 0.0031 & 0.4859 & 0.0127 & 12.063 & 0.381 & 0.1801 & 0.0032 & 0.831 & 2553 & 55 & 2654 & 29 & 4 \\
\hline 32.1 & 90 & 171 & 1.90 & 40.2 & 0.000147 & 0.20 & 1.929 & 0.034 & 0.1864 & 0.0017 & 0.5175 & 0.0092 & 13.174 & 0.292 & 0.1846 & 0.0025 & 0.801 & 2688 & 39 & 2695 & 22 & 0 \\
\hline 33.1 & 59 & 18 & 0.30 & 20.3 & - & $<0.01$ & 2.503 & 0.059 & 0.1880 & 0.0034 & 0.3996 & 0.0095 & 10.371 & 0.309 & 0.1882 & 0.0034 & 0.795 & 2167 & 44 & 2727 & 30 & 21 \\
\hline 34.1 & 130 & 49 & 0.38 & 58.4 & - & $<0.01$ & 1.908 & 0.030 & 0.1889 & 0.0015 & 0.5242 & 0.0082 & 13.658 & 0.239 & 0.1890 & 0.0015 & 0.894 & 2717 & 35 & 2733 & 13 & 1 \\
\hline 34.2 & 61 & 36 & 0.60 & 14.2 & - & $<0.01$ & 3.667 & 0.163 & 0.1241 & 0.0098 & 0.2727 & 0.0121 & 4.668 & 0.423 & 0.1241 & 0.0098 & 0.492 & 1555 & 62 & 2017 & 140 & 23 \\
\hline 35.2 & 504 & 505 & 1.00 & 95.1 & 0.001359 & 2.09 & 4.559 & 0.066 & 0.1345 & 0.0036 & 0.2148 & 0.0032 & 3.441 & 0.174 & 0.1162 & 0.0056 & 0.298 & 1254 & 17 & 1899 & 87 & 34 \\
\hline 36.1 & 133 & 99 & 0.74 & 56.2 & 0.000000 & 0.00 & 2.027 & 0.056 & 0.1853 & 0.0043 & 0.4933 & 0.0137 & 12.601 & 0.455 & 0.1853 & 0.0043 & 0.769 & 2585 & 59 & 2701 & 38 & 4 \\
\hline 37.1 & 53 & 53 & 0.99 & 13.3 & 0.000310 & 0.48 & 3.436 & 0.102 & 0.1125 & 0.0030 & 0.2896 & 0.0088 & 4.324 & 0.275 & 0.1083 & 0.0060 & 0.478 & 1640 & 44 & 1770 & 102 & 7 \\
\hline 38.1 & 72 & 45 & 0.63 & 27.9 & 0.002583 & 3.61 & 2.219 & 0.061 & 0.2016 & 0.0049 & 0.4344 & 0.0122 & 10.147 & 0.584 & 0.1694 & 0.0085 & 0.490 & 2326 & 55 & 2552 & 84 & 9 \\
\hline 39.1 & 75 & 37 & 0.49 & 23.6 & 0.000307 & 0.50 & 2.739 & 0.070 & 0.0904 & 0.0176 & 0.3633 & 0.0093 & 4.312 & 0.897 & 0.0861 & 0.0178 & 0.123 & 1998 & 44 & 1340 & 399 & -49 \\
\hline 40.1 & 172 & 101 & 0.59 & 73.9 & 0.000134 & 0.18 & 2.003 & 0.040 & 0.1846 & 0.0020 & 0.4984 & 0.0100 & 12.575 & 0.290 & 0.1830 & 0.0021 & 0.871 & 2607 & 43 & 2680 & 19 & 3 \\
\hline 41.1 & 125 & 122 & 1.00 & 54.4 & 0.000442 & 0.60 & 1.977 & 0.042 & 0.1943 & 0.0029 & 0.5028 & 0.0106 & 13.101 & 0.353 & 0.1890 & 0.0032 & 0.785 & 2626 & 46 & 2733 & 28 & 4 \\
\hline 42.1 & 68 & 45 & 0.66 & 21.6 & 0.000673 & 1.06 & 2.728 & 0.061 & 0.1143 & 0.0024 & 0.3626 & 0.0083 & 5.254 & 0.287 & 0.1051 & 0.0052 & 0.419 & 1995 & 39 & 1716 & 91 & -16 \\
\hline 43.1 & 86 & 63 & 0.74 & 23.9 & - & $<0.01$ & 3.087 & 0.066 & 0.1156 & 0.0019 & 0.3243 & 0.0070 & 5.220 & 0.144 & 0.1167 & 0.0020 & 0.780 & 1811 & 34 & 1907 & 31 & 5 \\
\hline 44.1 & 37 & 38 & 1.00 & 10.1 & 0.000595 & 0.93 & 3.182 & 0.085 & 0.1163 & 0.0028 & 0.3114 & 0.0084 & 4.645 & 0.221 & 0.1082 & 0.0042 & 0.569 & 1747 & 41 & 1769 & 72 & 1 \\
\hline 45.1 & 141 & 278 & 1.97 & 40.3 & 0.000178 & 0.28 & 3.010 & 0.048 & 0.1152 & 0.0017 & 0.3313 & 0.0053 & 5.155 & 0.118 & 0.1128 & 0.0019 & 0.697 & 1845 & 26 & 1845 & 30 & 0 \\
\hline 46.1 & 73 & 62 & 0.85 & 21.2 & 0.000721 & 1.14 & 2.949 & 0.073 & 0.1137 & 0.0021 & 0.3398 & 0.0085 & 5.396 & 0.167 & 0.1152 & 0.0021 & 0.806 & 1886 & 46 & 1883 & 33 & 0 \\
\hline 48.1 & 56 & 63 & 1.12 & 14.9 & 0.000243 & 0.38 & 3.235 & 0.075 & 0.1095 & 0.0020 & 0.3079 & 0.0071 & 4.509 & 0.152 & 0.1062 & 0.0026 & 0.686 & 1730 & 35 & 1735 & 45 & 0 \\
\hline 49.1 & 136 & 268 & 1.96 & 37.5 & 0.000073 & 0.11 & 3.125 & 0.051 & 0.1106 & 0.0013 & 0.3196 & 0.0052 & 4.832 & 0.115 & 0.1096 & 0.0019 & 0.691 & 1788 & 26 & 1794 & 31 & 0 \\
\hline 50.1 & 641 & 143 & 0.22 & 139.6 & 0.000424 & 0.66 & 3.945 & 0.047 & 0.1146 & 0.0007 & 0.2518 & 0.0030 & 3.780 & 0.065 & 0.1089 & 0.0014 & 0.688 & 1448 & 15 & 1781 & 23 & 19 \\
\hline 51.1 & 93 & 89 & 0.95 & 26.5 & 0.000071 & 0.11 & 3.025 & 0.054 & 0.1093 & 0.0013 & 0.3310 & 0.0059 & 5.025 & 0.108 & 0.1101 & 0.0013 & 0.825 & 1843 & 33 & 1801 & 22 & -2 \\
\hline 52.1 & 267 & 179 & 0.67 & 93.9 & 0.000137 & 0.19 & 2.443 & 0.032 & 0.1816 & 0.0010 & 0.4086 & 0.0054 & 10.140 & 0.152 & 0.1800 & 0.0013 & 0.885 & 2209 & 25 & 2653 & 12 & 17 \\
\hline 53.1 & 49 & 52 & 1.05 & 13.9 & 0.000302 & 0.48 & 3.054 & 0.072 & 0.1100 & 0.0021 & 0.3280 & 0.0077 & 5.043 & 0.153 & 0.1115 & 0.0021 & 0.776 & 1829 & 44 & 1824 & 35 & 0 \\
\hline 54.1 & 35 & 53 & 1.53 & 9.8 & 0.000415 & 0.66 & 3.041 & 0.085 & 0.1068 & 0.0023 & 0.3314 & 0.0092 & 5.162 & 0.182 & 0.1130 & 0.0025 & 0.788 & 1845 & 56 & 1848 & 39 & 0 \\
\hline 55.1 & 41 & 47 & 1.15 & 11.1 & 0.000064 & 0.10 & 3.139 & 0.077 & 0.1143 & 0.0022 & 0.3183 & 0.0078 & 4.980 & 0.167 & 0.1135 & 0.0026 & 0.732 & 1781 & 38 & 1856 & 41 & 4 \\
\hline 56.1 & 178 & 64 & 0.36 & 82.3 & 0.000002 & 0.00 & 1.856 & 0.027 & 0.1874 & 0.0012 & 0.5389 & 0.0079 & 13.921 & 0.221 & 0.1874 & 0.0012 & 0.918 & 2779 & 33 & 2719 & 10 & -2 \\
\hline 57.1 & 26 & 31 & 1.21 & 7.1 & 0.001608 & 2.54 & 3.096 & 0.108 & 0.1245 & 0.0037 & 0.3205 & 0.0112 & 5.231 & 0.249 & 0.1184 & 0.0038 & 0.733 & 1792 & 67 & 1932 & 58 & 7 \\
\hline 58.1 & 16 & 14 & 0.88 & 4.6 & 0.001824 & 2.95 & 2.888 & 0.116 & 0.1180 & 0.0038 & 0.3484 & 0.0140 & 5.900 & 0.299 & 0.1228 & 0.0038 & 0.794 & 1927 & 76 & 1998 & 55 & 4 \\
\hline 59.1 & 96 & 78 & 0.81 & 40.2 & 0.000319 & 0.44 & 2.048 & 0.040 & 0.1823 & 0.0032 & 0.4862 & 0.0094 & 11.959 & 0.338 & 0.1784 & 0.0037 & 0.686 & 2554 & 41 & 2638 & 34 & 3 \\
\hline 60.1 & 69 & 98 & 1.41 & 19.0 & 0.000461 & 0.73 & 3.133 & 0.067 & 0.1095 & 0.0018 & 0.3272 & 0.0070 & 5.829 & 0.186 & 0.1292 & 0.0030 & 0.675 & 1825 & 42 & 2087 & 41 & 13 \\
\hline
\end{tabular}

percentage of ${ }^{206} \mathrm{~Pb}$ that is common Pb. Correction for common Pb made using the measured ${ }^{204} \mathrm{~Pb} /{ }^{206} \mathrm{~Pb}$ ratio. For \% Disc, $0 \%$ denotes a concordant analysis. $\mathrm{Pb} *$ is the proportion of $\mathrm{Pb}$ that is radiogenic. 
Table 5

Summary of SHRIMP U-Pb zircon results for Ljusnan river sand

\begin{tabular}{|c|c|c|c|c|c|c|c|c|c|c|c|c|c|c|c|c|c|c|c|c|c|c|}
\hline \multirow[b]{2}{*}{$\begin{array}{l}\text { Grain. } \\
\text { spot }\end{array}$} & \multirow[b]{2}{*}{$\begin{array}{l}\mathrm{U} \\
(\mathrm{ppm})\end{array}$} & \multirow[b]{2}{*}{$\begin{array}{l}\text { Th } \\
\text { (ppm) }\end{array}$} & \multirow[b]{2}{*}{$\mathrm{Th} / \mathrm{U}$} & \multirow[b]{2}{*}{$\begin{array}{l}\mathrm{Pb}^{*} \\
(\mathrm{ppm})\end{array}$} & \multirow[b]{2}{*}{${ }^{204} \mathrm{~Pb} /{ }^{206} \mathrm{~Pb}$} & \multirow[b]{2}{*}{$f_{206} \%$} & \multicolumn{4}{|l|}{ Total ratios } & \multicolumn{6}{|c|}{ Radiogenic ratios } & \multirow[b]{2}{*}{$\mathrm{r}$} & \multicolumn{4}{|l|}{ Age (Ma) } & \multirow[b]{2}{*}{ \% Disc } \\
\hline & & & & & & & ${ }^{238} \mathrm{U} /{ }^{206} \mathrm{~Pb}$ & \pm & ${ }^{207} \mathrm{~Pb} /{ }^{206} \mathrm{~Pb}$ & \pm & ${ }^{206} \mathrm{~Pb} /{ }^{238} \mathrm{U}$ & \pm & ${ }^{207} \mathrm{~Pb} /\left.\right|^{235} \mathrm{U}$ & \pm & ${ }^{207} \mathrm{~Pb} /{ }^{206} \mathrm{~Pb}$ & \pm & & ${ }^{206} \mathrm{~Pb} /{ }^{238} \mathrm{U}$ & \pm & ${ }^{207} \mathrm{~Pb} /{ }^{206} \mathrm{~Pb}$ & \pm & \\
\hline 1.1 & 158 & 103 & 0.65 & 40.4 & 0.000060 & 0.09 & 3.356 & 0.041 & 0.1038 & 0.0007 & 0.2977 & 0.0036 & 4.227 & 0.061 & 0.1030 & 0.0008 & 0.843 & 1680 & 18 & 1678 & 14 & 0 \\
\hline 2.1 & 226 & 178 & 0.79 & 59.2 & 0.000025 & 0.04 & 3.281 & 0.038 & 0.1039 & 0.0006 & 0.3046 & 0.0035 & 4.349 & 0.058 & 0.1035 & 0.0007 & 0.873 & 1714 & 17 & 1688 & 12 & -2 \\
\hline 3.1 & 215 & 132 & 0.61 & 56.4 & 0.000028 & 0.04 & 3.274 & 0.041 & 0.1035 & 0.0006 & 0.3053 & 0.0038 & 4.340 & 0.062 & 0.1031 & 0.0007 & 0.872 & 1718 & 19 & 1680 & 13 & -2 \\
\hline 4.1 & 24 & 15 & 0.63 & 6.1 & - & $<0.01$ & 3.351 & 0.069 & 0.1018 & 0.0019 & 0.2995 & 0.0062 & 4.328 & 0.145 & 0.1048 & 0.0027 & 0.622 & 1689 & 31 & 1711 & 48 & 1 \\
\hline 5.1 & 105 & 67 & 0.64 & 28.2 & 0.000055 & 0.09 & 3.195 & 0.042 & 0.1024 & 0.0009 & 0.3127 & 0.0041 & 4.383 & 0.069 & 0.1016 & 0.0009 & 0.831 & 1754 & 20 & 1654 & 16 & -6 \\
\hline 6.1 & 226 & 112 & 0.49 & 54.0 & 0.000024 & 0.04 & 3.594 & 0.042 & 0.1026 & 0.0009 & 0.2782 & 0.0033 & 3.924 & 0.058 & 0.1023 & 0.0009 & 0.797 & 1582 & 16 & 1666 & 16 & 5 \\
\hline 7.1 & 108 & 75 & 0.69 & 26.3 & - & $<0.01$ & 3.532 & 0.047 & 0.1037 & 0.0009 & 0.2833 & 0.0038 & 4.070 & 0.073 & 0.1042 & 0.0012 & 0.747 & 1608 & 19 & 1700 & 22 & 5 \\
\hline 8.1 & 944 & 314 & 0.33 & 154.7 & 0.001162 & 1.93 & 5.243 & 0.055 & 0.0983 & 0.0007 & 0.1870 & 0.0020 & 2.112 & 0.068 & 0.0819 & 0.0025 & 0.331 & 1105 & 11 & 1243 & 59 & 11 \\
\hline 9.1 & 385 & 193 & 0.50 & 79.0 & - & $<0.01$ & 4.190 & 0.047 & 0.1050 & 0.0006 & 0.2387 & 0.0027 & 3.460 & 0.044 & 0.1051 & 0.0006 & 0.892 & 1380 & 14 & 1716 & 10 & 20 \\
\hline 10.1 & 144 & 81 & 0.56 & 28.8 & 0.000072 & 0.11 & 4.303 & 0.055 & 0.1036 & 0.0009 & 0.2321 & 0.0030 & 3.285 & 0.052 & 0.1026 & 0.0010 & 0.801 & 1346 & 15 & 1672 & 18 & 20 \\
\hline 11.1 & 411 & 152 & 0.37 & 83.4 & 0.000218 & 0.35 & 4.229 & 0.046 & 0.1044 & 0.0005 & 0.2357 & 0.0026 & 3.296 & 0.046 & 0.1014 & 0.0009 & 0.785 & 1364 & 13 & 1650 & 16 & 17 \\
\hline 12.1 & 608 & 301 & 0.49 & 150.8 & 0.000022 & 0.04 & 3.460 & 0.037 & 0.1036 & 0.0004 & 0.2889 & 0.0031 & 4.114 & 0.047 & 0.1033 & 0.0004 & 0.938 & 1636 & 15 & 1684 & 7 & 3 \\
\hline 13.1 & 109 & 67 & 0.62 & 31.6 & 0.000003 & 0.00 & 2.956 & 0.041 & 0.1047 & 0.0009 & 0.3383 & 0.0046 & 4.881 & 0.081 & 0.1046 & 0.0010 & 0.830 & 1879 & 22 & 1708 & 17 & -10 \\
\hline 14.1 & 194 & 175 & 0.90 & 53.4 & 0.000021 & 0.03 & 3.123 & 0.037 & 0.1044 & 0.0007 & 0.3201 & 0.0038 & 4.594 & 0.063 & 0.1041 & 0.0007 & 0.877 & 1790 & 19 & 1698 & 12 & -5 \\
\hline 15.1 & 111 & 93 & 0.84 & 26.8 & - & $<0.01$ & 3.573 & 0.047 & 0.1054 & 0.0009 & 0.2800 & 0.0037 & 4.077 & 0.066 & 0.1056 & 0.0010 & 0.818 & 1591 & 19 & 1725 & 17 & 8 \\
\hline 16.1 & 92 & 58 & 0.63 & 23.8 & 0.000160 & 0.25 & 3.340 & 0.045 & 0.1049 & 0.0010 & 0.2987 & 0.0041 & 4.230 & 0.080 & 0.1027 & 0.0013 & 0.718 & 1685 & 20 & 1674 & 24 & -1 \\
\hline 17.1 & 50 & 29 & 0.58 & 13.7 & - & $<0.01$ & 3.144 & 0.051 & 0.1057 & 0.0013 & 0.3184 & 0.0051 & 4.673 & 0.099 & 0.1065 & 0.0014 & 0.766 & 1782 & 25 & 1740 & 25 & -2 \\
\hline 18.1 & 193 & 119 & 0.62 & 49.9 & 0.000020 & 0.03 & 3.331 & 0.040 & 0.1057 & 0.0007 & 0.3001 & 0.0036 & 4.363 & 0.059 & 0.1054 & 0.0007 & 0.879 & 1692 & 18 & 1722 & 12 & 2 \\
\hline 19.1 & 230 & 89 & 0.39 & 53.2 & 0.000011 & 0.02 & 3.707 & 0.043 & 0.1039 & 0.0007 & 0.2697 & 0.0031 & 3.859 & 0.052 & 0.1038 & 0.0007 & 0.866 & 1539 & 16 & 1692 & 12 & 9 \\
\hline 20.1 & 97 & 61 & 0.63 & 19.6 & 0.000104 & 0.17 & 4.259 & 0.058 & 0.0906 & 0.0010 & 0.2344 & 0.0032 & 2.881 & 0.054 & 0.0891 & 0.0011 & 0.727 & 1358 & 17 & 1407 & 24 & 4 \\
\hline 21.1 & 150 & 77 & 0.51 & 21.4 & 0.000003 & 0.01 & 6.005 & 0.070 & 0.0775 & 0.0009 & 0.1665 & 0.0020 & 1.779 & 0.030 & 0.0775 & 0.0009 & 0.697 & 993 & 11 & 1134 & 24 & 12 \\
\hline 22.1 & 417 & 247 & 0.59 & 100.4 & 0.000012 & 0.02 & 3.570 & 0.039 & 0.1030 & 0.0005 & 0.2800 & 0.0030 & 3.970 & 0.047 & 0.1028 & 0.0005 & 0.920 & 1592 & 15 & 1676 & 9 & 5 \\
\hline 23.1 & 350 & 192 & 0.55 & 89.6 & 0.000018 & 0.03 & 3.352 & 0.037 & 0.1028 & 0.0005 & 0.2983 & 0.0033 & 4.219 & 0.051 & 0.1026 & 0.0005 & 0.914 & 1683 & 16 & 1671 & 9 & -1 \\
\hline 24.1 & 137 & 69 & 0.51 & 23.6 & 0.000010 & 0.02 & 4.968 & 0.064 & 0.0768 & 0.0010 & 0.2012 & 0.0026 & 2.126 & 0.041 & 0.0766 & 0.0011 & 0.671 & 1182 & 14 & 1111 & 28 & -6 \\
\hline 25.1 & 161 & 74 & 0.46 & 29.8 & 0.000047 & 0.08 & 4.641 & 0.057 & 0.0791 & 0.0007 & 0.2153 & 0.0027 & 2.329 & 0.038 & 0.0784 & 0.0008 & 0.763 & 1257 & 14 & 1158 & 21 & -9 \\
\hline 26.1 & 436 & 136 & 0.31 & 105.2 & 0.000018 & 0.03 & 3.556 & 0.039 & 0.0935 & 0.0004 & 0.2811 & 0.0031 & 3.613 & 0.043 & 0.0932 & 0.0004 & 0.917 & 1597 & 15 & 1492 & 9 & -7 \\
\hline 27.1 & 243 & 187 & 0.77 & 70.2 & 0.000028 & 0.04 & 2.976 & 0.035 & 0.1041 & 0.0006 & 0.3359 & 0.0040 & 4.802 & 0.064 & 0.1037 & 0.0006 & 0.885 & 1867 & 19 & 1691 & 12 & -10 \\
\hline 28.1 & 130 & 77 & 0.59 & 35.7 & - & $<0.01$ & 3.129 & 0.047 & 0.1043 & 0.0012 & 0.3197 & 0.0048 & 4.609 & 0.089 & 0.1046 & 0.0013 & 0.777 & 1788 & 23 & 1707 & 22 & -5 \\
\hline 29.1 & 79 & 47 & 0.59 & 21.4 & 0.000013 & 0.02 & 3.172 & 0.049 & 0.0986 & 0.0012 & 0.3152 & 0.0049 & 4.278 & 0.084 & 0.0984 & 0.0012 & 0.784 & 1766 & 24 & 1595 & 23 & -11 \\
\hline
\end{tabular}




\begin{tabular}{|c|c|c|c|c|c|c|c|c|c|c|c|c|c|c|c|c|c|c|c|c|c|c|}
\hline 30.1 & 58 & 83 & 1.44 & 13.2 & 0.000024 & 0.04 & 3.759 & 0.059 & 0.0925 & 0.0013 & 0.2659 & 0.0042 & 3.381 & 0.073 & 0.0922 & 0.0014 & 0.732 & 1520 & 21 & 1472 & 28 & -3 \\
\hline 31.1 & 317 & 168 & 0.53 & 76.9 & 0.000015 & 0.02 & 3.547 & 0.040 & 0.1004 & 0.0006 & 0.2819 & 0.0032 & 3.896 & 0.050 & 0.1002 & 0.0006 & 0.894 & 1601 & 16 & 1629 & 11 & 2 \\
\hline 32.1 & 133 & 111 & 0.84 & 16.4 & 0.000071 & 0.12 & 6.958 & 0.100 & 0.0758 & 0.0020 & 0.1436 & 0.0021 & 1.480 & 0.050 & 0.0748 & 0.0023 & 0.428 & 865 & 12 & 1062 & 61 & 19 \\
\hline 33.1 & 405 & 243 & 0.60 & 94.7 & - & $<0.01$ & 3.675 & 0.040 & 0.1043 & 0.0005 & 0.2721 & 0.0030 & 3.916 & 0.047 & 0.1044 & 0.0005 & 0.915 & 1551 & 15 & 1703 & 9 & 9 \\
\hline 34.1 & 60 & 16 & 0.26 & 15.2 & - & $<0.01$ & 3.384 & 0.052 & 0.1008 & 0.0013 & 0.2955 & 0.0045 & 4.105 & 0.082 & 0.1008 & 0.0013 & 0.759 & 1669 & 22 & 1638 & 24 & -2 \\
\hline 35.1 & 76 & 26 & 0.35 & 10.3 & 0.000159 & 0.27 & 6.307 & 0.099 & 0.0743 & 0.0013 & 0.1581 & 0.0025 & 1.571 & 0.051 & 0.0720 & 0.0021 & 0.482 & 946 & 14 & 987 & 58 & 4 \\
\hline 36.1 & 176 & 79 & 0.45 & 47.2 & 0.000576 & 0.91 & 3.202 & 0.039 & 0.1113 & 0.0007 & 0.3095 & 0.0038 & 4.415 & 0.089 & 0.1035 & 0.0017 & 0.607 & 1738 & 19 & 1687 & 30 & -3 \\
\hline 37.1 & 483 & 357 & 0.74 & 137.5 & 0.000026 & 0.04 & 3.019 & 0.033 & 0.1044 & 0.0005 & 0.3311 & 0.0037 & 4.748 & 0.057 & 0.1040 & 0.0005 & 0.916 & 1844 & 18 & 1697 & 9 & -9 \\
\hline 38.1 & 57 & 40 & 0.70 & 15.5 & - & $<0.01$ & 3.157 & 0.049 & 0.1063 & 0.0013 & 0.3175 & 0.0050 & 4.744 & 0.106 & 0.1084 & 0.0017 & 0.699 & 1777 & 24 & 1772 & 29 & 0 \\
\hline 39.1 & 779 & 369 & 0.47 & 191.1 & 0.000038 & 0.06 & 3.503 & 0.037 & 0.1032 & 0.0004 & 0.2853 & 0.0030 & 4.040 & 0.046 & 0.1027 & 0.0004 & 0.929 & 1618 & 15 & 1674 & 8 & 3 \\
\hline 40.1 & 271 & 294 & 1.09 & 46.4 & 0.000017 & 0.03 & 5.013 & 0.062 & 0.0929 & 0.0008 & 0.1994 & 0.0025 & 2.548 & 0.039 & 0.0927 & 0.0009 & 0.795 & 1172 & 13 & 1481 & 18 & 21 \\
\hline 41.1 & 213 & 135 & 0.63 & 47.9 & 0.000008 & 0.01 & 3.825 & 0.045 & 0.1045 & 0.0007 & 0.2614 & 0.0031 & 3.763 & 0.052 & 0.1044 & 0.0007 & 0.859 & 1497 & 16 & 1704 & 13 & 12 \\
\hline 42.1 & 222 & 53 & 0.24 & 103.6 & 0.000042 & 0.06 & 1.844 & 0.021 & 0.1981 & 0.0007 & 0.5420 & 0.0062 & 14.763 & 0.177 & 0.1976 & 0.0007 & 0.952 & 2792 & 26 & 2806 & 6 & 1 \\
\hline 43.1 & 209 & 128 & 0.61 & 58.1 & 0.000024 & 0.04 & 3.092 & 0.038 & 0.1045 & 0.0007 & 0.3233 & 0.0040 & 4.642 & 0.066 & 0.1041 & 0.0007 & 0.877 & 1806 & 20 & 1699 & 13 & -6 \\
\hline 44.1 & 154 & 105 & 0.68 & 43.0 & 0.000019 & 0.03 & 3.080 & 0.038 & 0.1048 & 0.0008 & 0.3246 & 0.0040 & 4.678 & 0.067 & 0.1045 & 0.0008 & 0.858 & 1812 & 20 & 1706 & 14 & -6 \\
\hline 45.1 & 257 & 174 & 0.68 & 73.5 & 0.000028 & 0.04 & 3.007 & 0.035 & 0.1051 & 0.0006 & 0.3324 & 0.0039 & 4.799 & 0.063 & 0.1047 & 0.0006 & 0.883 & 1850 & 19 & 1709 & 11 & -8 \\
\hline 46.1 & 358 & 255 & 0.71 & 86.9 & 0.000142 & 0.22 & 3.539 & 0.040 & 0.1058 & 0.0005 & 0.2819 & 0.0032 & 4.037 & 0.060 & 0.1038 & 0.0010 & 0.753 & 1601 & 16 & 1694 & 18 & 5 \\
\hline 47.1 & 143 & 95 & 0.66 & 41.1 & 0.000020 & 0.03 & 3.001 & 0.038 & 0.1038 & 0.0008 & 0.3331 & 0.0042 & 4.755 & 0.072 & 0.1035 & 0.0009 & 0.830 & 1853 & 20 & 1688 & 16 & -10 \\
\hline 48.1 & 295 & 171 & 0.58 & 66.1 & 0.000175 & 0.28 & 3.832 & 0.042 & 0.1049 & 0.0005 & 0.2602 & 0.0029 & 3.678 & 0.050 & 0.1025 & 0.0008 & 0.810 & 1491 & 15 & 1670 & 15 & 11 \\
\hline 49.1 & 258 & 250 & 0.97 & 67.7 & 0.000010 & 0.02 & 3.274 & 0.039 & 0.1047 & 0.0006 & 0.3053 & 0.0036 & 4.404 & 0.059 & 0.1046 & 0.0006 & 0.885 & 1718 & 18 & 1707 & 11 & -1 \\
\hline 50.1 & 303 & 208 & 0.69 & 77.1 & 0.000041 & 0.07 & 3.374 & 0.038 & 0.1038 & 0.0006 & 0.2961 & 0.0034 & 4.215 & 0.054 & 0.1032 & 0.0006 & 0.886 & 1672 & 17 & 1683 & 11 & 1 \\
\hline 51.1 & 81 & 26 & 0.32 & 11.3 & - & $<0.01$ & 6.100 & 0.096 & 0.0685 & 0.0012 & 0.1642 & 0.0026 & 1.575 & 0.043 & 0.0696 & 0.0015 & 0.581 & 980 & 14 & 917 & 45 & -7 \\
\hline 52.1 & 293 & 185 & 0.63 & 75.2 & - & $<0.01$ & 3.344 & 0.039 & 0.1041 & 0.0006 & 0.2991 & 0.0034 & 4.297 & 0.056 & 0.1042 & 0.0006 & 0.891 & 1687 & 17 & 1700 & 11 & 1 \\
\hline 53.1 & 177 & 123 & 0.69 & 47.3 & 0.000014 & 0.02 & 3.211 & 0.040 & 0.1076 & 0.0008 & 0.3114 & 0.0039 & 4.610 & 0.068 & 0.1074 & 0.0008 & 0.852 & 1748 & 19 & 1755 & 14 & 0 \\
\hline 54.1 & 504 & 276 & 0.55 & 128.2 & 0.000037 & 0.06 & 3.376 & 0.038 & 0.1045 & 0.0004 & 0.2961 & 0.0033 & 4.245 & 0.051 & 0.1040 & 0.0005 & 0.920 & 1672 & 16 & 1696 & 9 & 1 \\
\hline 55.1 & 411 & 103 & 0.25 & 91.1 & 0.000019 & 0.03 & 3.882 & 0.043 & 0.0946 & 0.0005 & 0.2575 & 0.0028 & 3.349 & 0.041 & 0.0943 & 0.0005 & 0.899 & 1477 & 15 & 1514 & 10 & 2 \\
\hline 56.1 & 175 & 107 & 0.61 & 44.5 & - & $<0.01$ & 3.371 & 0.041 & 0.1041 & 0.0010 & 0.2968 & 0.0036 & 4.275 & 0.069 & 0.1045 & 0.0011 & 0.763 & 1675 & 18 & 1705 & 19 & 2 \\
\hline 57.1 & 197 & 175 & 0.89 & 49.7 & 0.000001 & $<0.01$ & 3.405 & 0.041 & 0.1031 & 0.0007 & 0.2937 & 0.0035 & 4.173 & 0.058 & 0.1031 & 0.0007 & 0.863 & 1660 & 18 & 1680 & 13 & 1 \\
\hline 58.1 & 61 & 23 & 0.37 & 7.1 & 0.000174 & 0.30 & 7.364 & 0.128 & 0.0676 & 0.0015 & 0.1358 & 0.0024 & 1.265 & 0.036 & 0.0676 & 0.0015 & 0.605 & 821 & 14 & 855 & 48 & 4 \\
\hline 59.1 & 334 & 175 & 0.52 & 86.5 & 0.000001 & $<0.01$ & 3.317 & 0.038 & 0.1045 & 0.0006 & 0.3015 & 0.0035 & 4.342 & 0.056 & 0.1044 & 0.0006 & 0.893 & 1699 & 17 & 1705 & 11 & 0 \\
\hline 60.1 & 91 & 65 & 0.71 & 24.6 & - & $<0.01$ & 3.191 & 0.046 & 0.1056 & 0.0011 & 0.3134 & 0.0045 & 4.562 & 0.080 & 0.1056 & 0.0011 & 0.816 & 1758 & 22 & 1724 & 19 & -2 \\
\hline 61.1 & 464 & 364 & 0.78 & 120.7 & 0.000037 & 0.06 & 3.300 & 0.036 & 0.1044 & 0.0005 & 0.3029 & 0.0033 & 4.337 & 0.053 & 0.1039 & 0.0006 & 0.898 & 1706 & 17 & 1694 & 10 & -1 \\
\hline
\end{tabular}

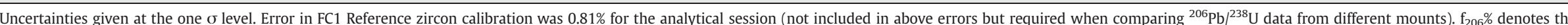
percentage of ${ }^{206} \mathrm{~Pb}$ that is common $\mathrm{Pb}$. Correction for common $\mathrm{Pb}$ made using the measured ${ }^{204} \mathrm{~Pb} /{ }^{206} \mathrm{~Pb}$ ratio. For \% Disc, $0 \%$ denotes a concordant analysis. Pb* is the proportion of $\mathrm{Pb}$ that is radiogenic. 
Table 6

Summary of SHRIMP U-Pb zircon results for Saltelva river sand

\begin{tabular}{|c|c|c|c|c|c|c|c|c|c|c|c|c|c|c|c|c|c|c|c|c|c|c|}
\hline \multirow[b]{2}{*}{$\begin{array}{l}\text { Grain. } \\
\text { spot }\end{array}$} & \multirow[b]{2}{*}{$\begin{array}{l}\mathrm{U} \\
(\mathrm{ppm})\end{array}$} & \multirow[b]{2}{*}{$\begin{array}{l}\text { Th } \\
(\mathrm{ppm})\end{array}$} & \multirow[b]{2}{*}{$\mathrm{Th} / \mathrm{U}$} & \multirow[b]{2}{*}{$\begin{array}{l}\mathrm{Pb}^{*} \\
(\mathrm{ppm})\end{array}$} & \multirow[b]{2}{*}{${ }^{204} \mathrm{~Pb} /{ }^{206} \mathrm{~Pb}$} & \multirow[b]{2}{*}{$f_{206} \%$} & \multicolumn{4}{|l|}{ Total ratios } & \multicolumn{6}{|c|}{ Radiogenic ratios } & \multirow[b]{2}{*}{$\mathrm{r}$} & \multicolumn{4}{|l|}{ Age (Ma) } & \multirow[b]{2}{*}{$\%$ Disc } \\
\hline & & & & & & & ${ }^{238} \mathrm{U} /{ }^{206} \mathrm{~Pb}$ & \pm & ${ }^{207} \mathrm{~Pb} /{ }^{206} \mathrm{~Pb}$ & \pm & ${ }^{206} \mathrm{~Pb} /{ }^{238} \mathrm{U}$ & \pm & ${ }^{207} \mathrm{~Pb} /\left.\right|^{235} \mathrm{U}$ & \pm & ${ }^{207} \mathrm{~Pb} /{ }^{206} \mathrm{~Pb}$ & \pm & & ${ }^{206} \mathrm{~Pb} /{ }^{238} \mathrm{U}$ & \pm & ${ }^{207} \mathrm{~Pb} /{ }^{206} \mathrm{~Pb}$ & \pm & \\
\hline 1.1 & 328 & 6 & 0.02 & 47.1 & - & $<0.01$ & 5.974 & 0.079 & 0.0736 & 0.0009 & 0.1674 & 0.0022 & 1.700 & 0.030 & 0.0736 & 0.0009 & 0.744 & 998 & 12 & 1032 & 24 & 3 \\
\hline 2.1 & 333 & 88 & 0.27 & 57.1 & 0.000025 & 0.04 & 5.013 & 0.065 & 0.0781 & 0.0008 & 0.1994 & 0.0026 & 2.138 & 0.037 & 0.0778 & 0.0009 & 0.759 & 1172 & 14 & 1141 & 22 & -3 \\
\hline 3.1 & 298 & 118 & 0.40 & 83.0 & 0.000009 & 0.01 & 3.084 & 0.040 & 0.1099 & 0.0008 & 0.3242 & 0.0042 & 4.908 & 0.075 & 0.1098 & 0.0009 & 0.860 & 1810 & 21 & 1796 & 14 & -1 \\
\hline 4.1 & 97 & 27 & 0.28 & 16.6 & 0.000086 & 0.15 & 5.017 & 0.095 & 0.0786 & 0.0016 & 0.1990 & 0.0038 & 2.124 & 0.059 & 0.0774 & 0.0016 & 0.679 & 1170 & 20 & 1131 & 41 & -3 \\
\hline 5.1 & 357 & 84 & 0.24 & 58.9 & 0.000189 & 0.30 & 5.208 & 0.081 & 0.0985 & 0.0011 & 0.1914 & 0.0030 & 2.530 & 0.064 & 0.0958 & 0.0019 & 0.615 & 1129 & 16 & 1545 & 38 & 27 \\
\hline 6.1 & 621 & 345 & 0.56 & 127.7 & 0.000292 & 0.47 & 4.180 & 0.057 & 0.0957 & 0.0009 & 0.2372 & 0.0033 & 2.896 & 0.057 & 0.0886 & 0.0012 & 0.699 & 1372 & 19 & 1394 & 27 & 2 \\
\hline 7.1 & 1474 & 189 & 0.13 & 225.9 & 0.000019 & 0.03 & 5.606 & 0.061 & 0.0748 & 0.0004 & 0.1783 & 0.0019 & 1.831 & 0.023 & 0.0745 & 0.0004 & 0.875 & 1058 & 11 & 1054 & 12 & 0 \\
\hline 8.1 & 416 & 181 & 0.44 & 71.0 & - & $<0.01$ & 5.025 & 0.064 & 0.0778 & 0.0007 & 0.1993 & 0.0025 & 2.169 & 0.042 & 0.0790 & 0.0012 & 0.657 & 1171 & 14 & 1171 & 29 & 0 \\
\hline 9.1 & 257 & 94 & 0.37 & 52.7 & 0.000039 & 0.06 & 4.183 & 0.058 & 0.0870 & 0.0009 & 0.2389 & 0.0033 & 2.846 & 0.053 & 0.0864 & 0.0011 & 0.745 & 1381 & 17 & 1347 & 24 & -2 \\
\hline 10.1 & 202 & 24 & 0.12 & 51.4 & 0.000076 & 0.12 & 3.367 & 0.058 & 0.1038 & 0.0011 & 0.2966 & 0.0051 & 4.202 & 0.094 & 0.1028 & 0.0015 & 0.764 & 1674 & 25 & 1675 & 27 & 0 \\
\hline 11.1 & 336 & 172 & 0.51 & 142.1 & 0.000034 & 0.05 & 2.033 & 0.026 & 0.1902 & 0.0009 & 0.4916 & 0.0062 & 12.866 & 0.173 & 0.1898 & 0.0009 & 0.938 & 2578 & 27 & 2740 & 8 & 6 \\
\hline 12.1 & 59 & 41 & 0.69 & 12.8 & 0.000565 & 0.93 & 3.963 & 0.090 & 0.0930 & 0.0020 & 0.2522 & 0.0057 & 3.219 & 0.101 & 0.0926 & 0.0020 & 0.722 & 1450 & 33 & 1479 & 41 & 2 \\
\hline 13.1 & 171 & 104 & 0.61 & 44.7 & 0.000104 & 0.17 & 3.290 & 0.049 & 0.1009 & 0.0010 & 0.3035 & 0.0045 & 4.164 & 0.078 & 0.0995 & 0.0011 & 0.795 & 1709 & 22 & 1615 & 21 & -6 \\
\hline 15.1 & 42 & 36 & 0.87 & 10.4 & 0.000917 & 1.46 & 3.414 & 0.100 & 0.1138 & 0.0030 & 0.2887 & 0.0087 & 4.027 & 0.311 & 0.1012 & 0.0072 & 0.389 & 1635 & 43 & 1646 & 132 & 1 \\
\hline 16.1 & 931 & 276 & 0.30 & 250.0 & 0.000023 & 0.03 & 3.200 & 0.038 & 0.1494 & 0.0006 & 0.3124 & 0.0038 & 6.421 & 0.082 & 0.1491 & 0.0006 & 0.941 & 1753 & 18 & 2335 & 7 & 25 \\
\hline 17.1 & 47 & 18 & 0.37 & 8.1 & - & $<0.01$ & 4.981 & 0.133 & 0.0841 & 0.0026 & 0.1995 & 0.0053 & 2.167 & 0.095 & 0.0788 & 0.0027 & 0.612 & 1173 & 31 & 1167 & 68 & -1 \\
\hline 18.1 & 95 & 56 & 0.59 & 22.7 & - & $<0.01$ & 3.579 & 0.066 & 0.0961 & 0.0015 & 0.2800 & 0.0053 & 3.774 & 0.141 & 0.0978 & 0.0032 & 0.503 & 1591 & 27 & 1582 & 61 & -1 \\
\hline 19.1 & 483 & 124 & 0.26 & 87.7 & 0.000623 & 1.02 & 4.730 & 0.059 & 0.0948 & 0.0011 & 0.2086 & 0.0026 & 2.406 & 0.059 & 0.0836 & 0.0018 & 0.509 & 1221 & 15 & 1284 & 41 & 5 \\
\hline 20.1 & 122 & 37 & 0.30 & 17.7 & 0.000299 & 0.51 & 5.904 & 0.102 & 0.0752 & 0.0014 & 0.1694 & 0.0029 & 1.763 & 0.045 & 0.0755 & 0.0014 & 0.677 & 1009 & 17 & 1081 & 38 & 7 \\
\hline 21.1 & 168 & 78 & 0.47 & 46.2 & 0.000024 & 0.04 & 3.123 & 0.047 & 0.1093 & 0.0010 & 0.3201 & 0.0048 & 4.810 & 0.087 & 0.1090 & 0.0011 & 0.835 & 1790 & 24 & 1783 & 18 & 0 \\
\hline 22.1 & 109 & 33 & 0.31 & 31.8 & 0.000103 & 0.16 & 2.944 & 0.051 & 0.1122 & 0.0013 & 0.3399 & 0.0059 & 5.282 & 0.111 & 0.1127 & 0.0013 & 0.832 & 1886 & 30 & 1843 & 21 & -2 \\
\hline 23.1 & 55 & 55 & 1.00 & 8.6 & 0.000136 & 0.23 & 5.475 & 0.129 & 0.0784 & 0.0048 & 0.1822 & 0.0046 & 1.921 & 0.224 & 0.0765 & 0.0087 & 0.215 & 1079 & 25 & 1107 & 227 & 3 \\
\hline 24.1 & 147 & 72 & 0.49 & 29.9 & - & $<0.01$ & 4.218 & 0.066 & 0.0932 & 0.0012 & 0.2374 & 0.0037 & 3.089 & 0.062 & 0.0944 & 0.0012 & 0.781 & 1373 & 19 & 1516 & 24 & 9 \\
\hline 25.1 & 1214 & 324 & 0.27 & 293.8 & 0.000051 & 0.08 & 3.550 & 0.040 & 0.0988 & 0.0004 & 0.2815 & 0.0032 & 3.808 & 0.047 & 0.0981 & 0.0005 & 0.915 & 1599 & 16 & 1589 & 9 & -1 \\
\hline 26.1 & 330 & 124 & 0.37 & 87.5 & 0.000007 & 0.01 & 3.239 & 0.041 & 0.1097 & 0.0008 & 0.3087 & 0.0039 & 4.663 & 0.071 & 0.1096 & 0.0009 & 0.842 & 1734 & 19 & 1792 & 15 & 3 \\
\hline 27.1 & 383 & 196 & 0.51 & 84.5 & 0.000025 & 0.04 & 3.893 & 0.049 & 0.0935 & 0.0007 & 0.2568 & 0.0032 & 3.297 & 0.049 & 0.0931 & 0.0007 & 0.847 & 1473 & 16 & 1490 & 15 & 1 \\
\hline 29.1 & 74 & 53 & 0.71 & 17.9 & 0.000331 & 0.54 & 3.578 & 0.075 & 0.0965 & 0.0016 & 0.2807 & 0.0058 & 3.863 & 0.103 & 0.0998 & 0.0017 & 0.778 & 1595 & 33 & 1621 & 31 & 2 \\
\hline 30.1 & 103 & 41 & 0.40 & 15.6 & 0.000214 & 0.37 & 5.667 & 0.103 & 0.0750 & 0.0014 & 0.1758 & 0.0032 & 1.745 & 0.063 & 0.0720 & 0.0022 & 0.506 & 1044 & 18 & 985 & 64 & -6 \\
\hline 31.1 & 145 & 35 & 0.24 & 18.1 & 0.006774 & 12.00 & 6.863 & 0.104 & 0.1585 & 0.0019 & 0.1282 & 0.0026 & 1.066 & 0.313 & 0.0603 & 0.0177 & 0.069 & 778 & 15 & 615 & 633 & -27 \\
\hline 32.1 & 173 & 47 & 0.27 & 27.0 & 0.000072 & 0.12 & 5.519 & 0.078 & 0.0760 & 0.0010 & 0.1810 & 0.0026 & 1.870 & 0.041 & 0.0749 & 0.0012 & 0.652 & 1072 & 14 & 1067 & 33 & -1 \\
\hline 33.1 & 209 & 109 & 0.52 & 27.6 & 0.000212 & 0.35 & 6.526 & 0.103 & 0.0927 & 0.0060 & 0.1527 & 0.0024 & 1.889 & 0.132 & 0.0897 & 0.0061 & 0.225 & 916 & 13 & 1420 & 130 & 35 \\
\hline 34.1 & 187 & 70 & 0.37 & 40.9 & 0.000162 & 0.27 & 3.925 & 0.052 & 0.0891 & 0.0009 & 0.2550 & 0.0034 & 3.155 & 0.052 & 0.0897 & 0.0009 & 0.814 & 1464 & 18 & 1420 & 18 & -3 \\
\hline 35.1 & 593 & 194 & 0.33 & 122.4 & 0.000064 & 0.10 & 4.162 & 0.047 & 0.1050 & 0.0005 & 0.2401 & 0.0027 & 3.447 & 0.045 & 0.1041 & 0.0007 & 0.864 & 1387 & 14 & 1699 & 12 & 18 \\
\hline
\end{tabular}




\begin{tabular}{|c|c|c|c|c|c|c|c|c|c|c|c|c|c|c|c|c|c|c|c|c|c|c|}
\hline 36.1 & 257 & 186 & 0.72 & 74.1 & 0.000020 & 0.03 & 2.981 & 0.039 & 0.1114 & 0.0008 & 0.3354 & 0.0044 & 5.137 & 0.081 & 0.1111 & 0.0010 & 0.823 & 1864 & 21 & 1817 & 16 & -3 \\
\hline 37.1 & 185 & 61 & 0.33 & 32.2 & 0.000258 & 0.44 & 4.917 & 0.071 & 0.0777 & 0.0010 & 0.2035 & 0.0029 & 2.198 & 0.042 & 0.0783 & 0.0010 & 0.761 & 1194 & 16 & 1155 & 24 & -3 \\
\hline 38.1 & 605 & 139 & 0.23 & 160.0 & 0.000011 & 0.02 & 3.247 & 0.037 & 0.1009 & 0.0005 & 0.3079 & 0.0035 & 4.278 & 0.052 & 0.1008 & 0.0005 & 0.920 & 1730 & 17 & 1638 & 9 & -6 \\
\hline 39.1 & 159 & 78 & 0.49 & 41.6 & 0.000044 & 0.07 & 3.293 & 0.047 & 0.1034 & 0.0010 & 0.3035 & 0.0043 & 4.303 & 0.078 & 0.1028 & 0.0012 & 0.782 & 1709 & 21 & 1676 & 21 & -2 \\
\hline 40.1 & 142 & 60 & 0.42 & 25.9 & 0.000130 & 0.22 & 4.717 & 0.069 & 0.0832 & 0.0011 & 0.2115 & 0.0031 & 2.373 & 0.070 & 0.0814 & 0.0021 & 0.499 & 1237 & 17 & 1230 & 50 & -1 \\
\hline 41.1 & 95 & 111 & 1.16 & 13.5 & 0.000311 & 0.54 & 6.045 & 0.119 & 0.0704 & 0.0015 & 0.1660 & 0.0033 & 1.684 & 0.049 & 0.0735 & 0.0016 & 0.675 & 990 & 22 & 1029 & 44 & 4 \\
\hline 42.1 & 39 & 17 & 0.45 & 6.9 & 0.001645 & 2.81 & 4.829 & 0.311 & 0.0953 & 0.0049 & 0.2067 & 0.0133 & 2.664 & 0.221 & 0.0935 & 0.0049 & 0.777 & 1211 & 76 & 1498 & 99 & 19 \\
\hline 43.1 & 180 & 75 & 0.42 & 34.7 & 0.000373 & 0.61 & 4.463 & 0.062 & 0.0960 & 0.0010 & 0.2227 & 0.0031 & 2.789 & 0.066 & 0.0908 & 0.0017 & 0.586 & 1296 & 16 & 1443 & 37 & 10 \\
\hline 44.1 & 133 & 46 & 0.30 & 36.6 & 0.000129 & 0.20 & 3.126 & 0.045 & 0.1112 & 0.0010 & 0.3193 & 0.0046 & 4.817 & 0.098 & 0.1094 & 0.0016 & 0.715 & 1786 & 23 & 1790 & 26 & 0 \\
\hline 45.1 & 90 & 44 & 0.49 & 16.8 & 0.000112 & 0.18 & 4.581 & 0.073 & 0.0903 & 0.0013 & 0.2179 & 0.0035 & 2.666 & 0.087 & 0.0887 & 0.0025 & 0.490 & 1271 & 19 & 1399 & 55 & 9 \\
\hline 46.1 & 101 & 35 & 0.35 & 18.4 & 0.000460 & 0.79 & 4.719 & 0.075 & 0.0790 & 0.0012 & 0.2118 & 0.0034 & 2.290 & 0.050 & 0.0784 & 0.0012 & 0.728 & 1238 & 19 & 1158 & 30 & -7 \\
\hline 47.1 & 189 & 140 & 0.74 & 25.9 & 0.000054 & 0.09 & 6.251 & 0.086 & 0.0725 & 0.0009 & 0.1598 & 0.0022 & 1.582 & 0.041 & 0.0718 & 0.0016 & 0.537 & 956 & 12 & 979 & 44 & 2 \\
\hline 48.1 & 147 & 54 & 0.37 & 32.6 & 0.000075 & 0.12 & 3.862 & 0.054 & 0.0966 & 0.0010 & 0.2586 & 0.0036 & 3.409 & 0.067 & 0.0956 & 0.0013 & 0.715 & 1483 & 19 & 1540 & 26 & 4 \\
\hline 49.1 & 49 & 31 & 0.63 & 14.6 & 0.000410 & 0.64 & 2.898 & 0.055 & 0.1125 & 0.0016 & 0.3451 & 0.0066 & 5.356 & 0.127 & 0.1126 & 0.0016 & 0.806 & 1911 & 35 & 1841 & 25 & -4 \\
\hline 50.1 & 235 & 90 & 0.38 & 38.7 & 0.000003 & 0.00 & 5.221 & 0.067 & 0.0759 & 0.0008 & 0.1915 & 0.0025 & 2.004 & 0.034 & 0.0759 & 0.0009 & 0.751 & 1130 & 13 & 1092 & 23 & -3 \\
\hline 51.1 & 407 & 180 & 0.44 & 110.5 & - & $<0.01$ & 3.162 & 0.037 & 0.1099 & 0.0006 & 0.3163 & 0.0037 & 4.797 & 0.063 & 0.1100 & 0.0007 & 0.887 & 1772 & 18 & 1799 & 11 & 2 \\
\hline 52.1 & 228 & 88 & 0.39 & 34.7 & 0.000034 & 0.06 & 5.641 & 0.073 & 0.0746 & 0.0008 & 0.1773 & 0.0023 & 1.832 & 0.030 & 0.0749 & 0.0008 & 0.773 & 1052 & 13 & 1066 & 21 & 1 \\
\hline 53.1 & 1315 & 225 & 0.17 & 294.8 & 0.000015 & 0.02 & 3.832 & 0.041 & 0.0926 & 0.0003 & 0.2609 & 0.0028 & 3.323 & 0.038 & 0.0924 & 0.0004 & 0.940 & 1494 & 14 & 1475 & 7 & -1 \\
\hline 54.1 & 62 & 42 & 0.68 & 10.4 & 0.000373 & 0.63 & 5.155 & 0.095 & 0.0791 & 0.0016 & 0.1940 & 0.0036 & 2.119 & 0.057 & 0.0792 & 0.0016 & 0.684 & 1143 & 22 & 1177 & 39 & 3 \\
\hline 55.1 & 299 & 121 & 0.40 & 48.0 & 0.000081 & 0.14 & 5.352 & 0.067 & 0.0760 & 0.0007 & 0.1870 & 0.0023 & 1.979 & 0.031 & 0.0767 & 0.0007 & 0.799 & 1105 & 13 & 1114 & 19 & 1 \\
\hline 56.1 & 745 & 20 & 0.03 & 52.8 & 0.000000 & 0.00 & 12.104 & 0.136 & 0.0578 & 0.0006 & 0.0826 & 0.0009 & 0.658 & 0.010 & 0.0578 & 0.0006 & 0.743 & 512 & 6 & 521 & 22 & 2 \\
\hline 57.1 & 53 & 60 & 1.12 & 14.4 & 0.000468 & 0.75 & 3.192 & 0.059 & 0.1034 & 0.0015 & 0.3131 & 0.0058 & 4.449 & 0.105 & 0.1030 & 0.0015 & 0.783 & 1756 & 34 & 1680 & 27 & -5 \\
\hline 58.1 & 12 & 8 & 0.67 & 5.2 & 0.001118 & 1.54 & 1.910 & 0.062 & 0.1927 & 0.0037 & 0.5179 & 0.0167 & 13.193 & 0.510 & 0.1848 & 0.0039 & 0.837 & 2690 & 78 & 2696 & 35 & 0 \\
\hline 59.1 & 155 & 38 & 0.25 & 43.0 & - & $<0.01$ & 3.101 & 0.042 & 0.1080 & 0.0009 & 0.3226 & 0.0043 & 4.820 & 0.081 & 0.1083 & 0.0011 & 0.802 & 1803 & 21 & 1772 & 18 & -2 \\
\hline 60.1 & 63 & 52 & 0.82 & 10.0 & 0.000002 & 0.00 & 5.464 & 0.098 & 0.0746 & 0.0015 & 0.1830 & 0.0033 & 1.883 & 0.055 & 0.0746 & 0.0017 & 0.611 & 1083 & 18 & 1058 & 47 & -2 \\
\hline
\end{tabular}

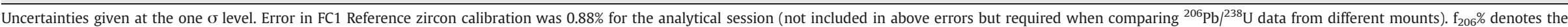
percentage of ${ }^{206} \mathrm{~Pb}$ that is common $\mathrm{Pb}$. Correction for common $\mathrm{Pb}$ made using the measured ${ }^{204} \mathrm{~Pb} /{ }^{206} \mathrm{~Pb}$ ratio. For \% Disc, $0 \%$ denotes a concordant analysis. Pb* is the proportion of $\mathrm{Pb}$ that is radiogenic. 
Table 7

Summary of SHRIMP U-Pb zircon results for Vefsna river sand

\begin{tabular}{|c|c|c|c|c|c|c|c|c|c|c|c|c|c|c|c|c|c|c|c|c|c|c|}
\hline \multirow[b]{2}{*}{$\begin{array}{l}\text { Grain. } \\
\text { spot }\end{array}$} & \multirow[b]{2}{*}{$\begin{array}{l}\mathrm{U} \\
(\mathrm{ppm})\end{array}$} & \multirow[b]{2}{*}{$\begin{array}{l}\text { Th } \\
\text { (ppm) }\end{array}$} & \multirow[b]{2}{*}{$\mathrm{Th} / \mathrm{U}$} & \multirow[b]{2}{*}{$\begin{array}{l}\mathrm{Pb}^{*} \\
(\mathrm{ppm})\end{array}$} & \multirow[b]{2}{*}{${ }^{204} \mathrm{~Pb} /{ }^{206} \mathrm{~Pb}$} & \multirow[b]{2}{*}{$\mathrm{f}_{206} \%$} & \multicolumn{4}{|l|}{ Total ratios } & \multicolumn{6}{|c|}{ Radiogenic ratios } & \multirow[b]{2}{*}{$\mathrm{r}$} & \multicolumn{4}{|l|}{ Age (Ma) } & \multirow[b]{2}{*}{ \% Disc } \\
\hline & & & & & & & ${ }^{238} \mathrm{U} / 206 \mathrm{~Pb}$ & \pm & ${ }^{207} \mathrm{~Pb} /{ }^{206} \mathrm{~Pb}$ & \pm & ${ }^{206} \mathrm{~Pb} /{ }^{238} \mathrm{U}$ & \pm & ${ }^{207} \mathrm{~Pb} /{ }^{235} \mathrm{U}$ & \pm & ${ }^{207} \mathrm{~Pb} /{ }^{206} \mathrm{~Pb}$ & \pm & & ${ }^{206} \mathrm{~Pb} / /^{238} \mathrm{U}$ & \pm & ${ }^{207} \mathrm{~Pb} /{ }^{206} \mathrm{~Pb}$ & \pm & \\
\hline 1.1 & 190 & 238 & 1.25 & 60.1 & 0.000060 & 0.09 & 3.399 & 0.045 & 0.1037 & 0.0009 & 0.2939 & 0.0039 & 4.170 & 0.068 & 0.1029 & 0.0010 & 0.821 & 1661 & 20 & 1677 & 17 & 1 \\
\hline 2.1 & 67 & 209 & 3.10 & 36.4 & 0.000036 & 0.06 & 4.927 & 0.069 & 0.0764 & 0.0009 & 0.2030 & 0.0028 & 2.147 & 0.039 & 0.0767 & 0.0009 & 0.766 & 1192 & 16 & 1113 & 23 & -7 \\
\hline 3.1 & 26 & 42 & 1.62 & 5.9 & 0.001040 & 1.84 & 6.129 & 0.159 & 0.0759 & 0.0025 & 0.1624 & 0.0042 & 1.613 & 0.071 & 0.0720 & 0.0026 & 0.590 & 970 & 26 & 987 & 72 & 2 \\
\hline 4.1 & 34 & 34 & 1.00 & 14.0 & 0.000147 & 0.21 & 2.073 & 0.053 & 0.1649 & 0.0024 & 0.4820 & 0.0122 & 10.924 & 0.321 & 0.1644 & 0.0024 & 0.863 & 2536 & 60 & 2501 & 25 & -1 \\
\hline 5.1 & 749 & 2445 & 3.27 & 141.5 & 0.000934 & 1.67 & 14.842 & 0.156 & 0.0708 & 0.0004 & 0.0662 & 0.0007 & 0.523 & 0.021 & 0.0572 & 0.0022 & 0.267 & 414 & 4 & 500 & 84 & 17 \\
\hline 6.1 & 151 & 624 & 4.13 & 113.6 & 0.000335 & 0.53 & 4.717 & 0.054 & 0.1063 & 0.0006 & 0.2109 & 0.0024 & 2.956 & 0.047 & 0.1017 & 0.0011 & 0.718 & 1233 & 13 & 1655 & 21 & 25 \\
\hline 7.1 & 56 & 121 & 2.17 & 18.7 & 0.000235 & 0.40 & 5.549 & 0.091 & 0.0751 & 0.0013 & 0.1795 & 0.0030 & 1.774 & 0.078 & 0.0717 & 0.0029 & 0.379 & 1064 & 16 & 977 & 83 & -9 \\
\hline 8.1 & 50 & 192 & 3.86 & 52.7 & 0.000063 & 0.10 & 3.122 & 0.043 & 0.1078 & 0.0009 & 0.3200 & 0.0044 & 4.717 & 0.080 & 0.1069 & 0.0011 & 0.812 & 1789 & 22 & 1748 & 18 & -2 \\
\hline 9.1 & 176 & 716 & 4.06 & 96.2 & 0.000690 & 1.11 & 6.393 & 0.074 & 0.1044 & 0.0007 & 0.1547 & 0.0018 & 2.024 & 0.050 & 0.0949 & 0.0020 & 0.476 & 927 & 10 & 1526 & 41 & 39 \\
\hline 10.1 & 21 & 81 & 3.85 & 15.2 & 0.000806 & 1.32 & 4.587 & 0.083 & 0.1001 & 0.0017 & 0.2151 & 0.0040 & 2.636 & 0.132 & 0.0889 & 0.0041 & 0.372 & 1256 & 21 & 1401 & 89 & 10 \\
\hline 11.1 & 18 & 34 & 1.92 & 10.2 & - & $<0.01$ & 2.866 & 0.071 & 0.1151 & 0.0023 & 0.3489 & 0.0087 & 5.539 & 0.178 & 0.1151 & 0.0023 & 0.773 & 1929 & 41 & 1882 & 37 & -3 \\
\hline 12.1 & 83 & 249 & 3.00 & 38.2 & 0.000074 & 0.13 & 5.589 & 0.075 & 0.0745 & 0.0009 & 0.1787 & 0.0024 & 1.809 & 0.043 & 0.0734 & 0.0014 & 0.571 & 1060 & 13 & 1025 & 39 & -3 \\
\hline 13.1 & 49 & 123 & 2.51 & 22.1 & 0.000307 & 0.52 & 4.762 & 0.075 & 0.0793 & 0.0012 & 0.2101 & 0.0033 & 2.312 & 0.050 & 0.0798 & 0.0012 & 0.733 & 1229 & 19 & 1192 & 29 & -3 \\
\hline 14.1 & 86 & 118 & 1.36 & 19.3 & 0.000050 & 0.08 & 5.234 & 0.087 & 0.0780 & 0.0014 & 0.1909 & 0.0032 & 2.035 & 0.069 & 0.0773 & 0.0023 & 0.492 & 1126 & 17 & 1129 & 59 & 0 \\
\hline 15.1 & 128 & 307 & 2.39 & 53.5 & 0.000165 & 0.28 & 4.940 & 0.068 & 0.0783 & 0.0007 & 0.2025 & 0.0028 & 2.197 & 0.037 & 0.0787 & 0.0007 & 0.829 & 1189 & 16 & 1164 & 18 & -2 \\
\hline 16.1 & 82 & 104 & 1.28 & 23.7 & - & $<0.01$ & 3.780 & 0.066 & 0.0948 & 0.0014 & 0.2645 & 0.0046 & 3.453 & 0.080 & 0.0947 & 0.0014 & 0.751 & 1513 & 26 & 1522 & 29 & 1 \\
\hline 17.1 & 138 & 216 & 1.57 & 57.7 & 0.000064 & 0.10 & 3.220 & 0.043 & 0.1024 & 0.0009 & 0.3103 & 0.0041 & 4.342 & 0.074 & 0.1015 & 0.0011 & 0.788 & 1742 & 20 & 1652 & 19 & -5 \\
\hline 18.1 & 283 & 640 & 2.26 & 134.8 & 0.000039 & 0.06 & 4.076 & 0.046 & 0.1067 & 0.0006 & 0.2452 & 0.0028 & 3.588 & 0.046 & 0.1061 & 0.0006 & 0.883 & 1414 & 14 & 1734 & 11 & 18 \\
\hline 19.1 & 51 & 73 & 1.45 & 9.2 & 0.000252 & 0.42 & 6.801 & 0.131 & 0.0820 & 0.0018 & 0.1464 & 0.0029 & 1.582 & 0.085 & 0.0784 & 0.0039 & 0.366 & 881 & 16 & 1157 & 99 & 24 \\
\hline 20.1 & 254 & 385 & 1.51 & 25.8 & - & $<0.01$ & 12.807 & 0.183 & 0.0556 & 0.0009 & 0.0782 & 0.0011 & 0.617 & 0.017 & 0.0572 & 0.0014 & 0.516 & 486 & 7 & 499 & 53 & 3 \\
\hline 21.1 & 148 & 334 & 2.26 & 99.3 & 0.000027 & 0.04 & 2.885 & 0.035 & 0.1101 & 0.0006 & 0.3465 & 0.0042 & 5.242 & 0.073 & 0.1097 & 0.0007 & 0.879 & 1918 & 20 & 1795 & 12 & -7 \\
\hline 22.1 & 303 & 367 & 1.21 & 25.2 & 0.000073 & 0.13 & 12.521 & 0.165 & 0.0573 & 0.0009 & 0.0798 & 0.0011 & 0.626 & 0.013 & 0.0569 & 0.0009 & 0.629 & 495 & 7 & 486 & 36 & -2 \\
\hline 23.1 & 105 & 168 & 1.60 & 46.4 & 0.000097 & 0.16 & 3.114 & 0.051 & 0.0881 & 0.0009 & 0.3228 & 0.0053 & 4.112 & 0.082 & 0.0924 & 0.0010 & 0.822 & 1804 & 29 & 1475 & 21 & -22 \\
\hline 24.1 & 148 & 506 & 3.43 & 130.7 & 0.000043 & 0.07 & 3.330 & 0.039 & 0.1101 & 0.0006 & 0.3001 & 0.0035 & 4.531 & 0.060 & 0.1095 & 0.0007 & 0.888 & 1692 & 17 & 1791 & 11 & 6 \\
\hline 25.1 & 21 & 40 & 1.93 & 6.9 & 0.003969 & 7.42 & 5.017 & 0.263 & 0.0971 & 0.0064 & 0.1966 & 0.0103 & 2.322 & 0.217 & 0.0857 & 0.0066 & 0.560 & 1157 & 62 & 1331 & 150 & 13 \\
\hline 26.1 & 263 & 268 & 1.02 & 18.0 & 0.000293 & 0.53 & 12.788 & 0.179 & 0.0577 & 0.0010 & 0.0784 & 0.0011 & 0.641 & 0.014 & 0.0593 & 0.0011 & 0.620 & 486 & 8 & 580 & 39 & 16 \\
\hline 27.1 & 104 & 398 & 3.81 & 58.5 & 0.000074 & 0.13 & 5.838 & 0.073 & 0.0772 & 0.0008 & 0.1706 & 0.0021 & 1.742 & 0.030 & 0.0741 & 0.0009 & 0.711 & 1016 & 12 & 1043 & 25 & 3 \\
\hline 28.1 & 413 & 1399 & 3.39 & 86.4 & 0.000074 & 0.13 & 13.911 & 0.157 & 0.0565 & 0.0005 & 0.0719 & 0.0008 & 0.556 & 0.008 & 0.0561 & 0.0005 & 0.813 & 447 & 5 & 455 & 18 & 2 \\
\hline 29.1 & 83 & 211 & 2.55 & 48.1 & 0.000311 & 0.50 & 3.772 & 0.050 & 0.1025 & 0.0009 & 0.2638 & 0.0035 & 3.571 & 0.077 & 0.0982 & 0.0017 & 0.621 & 1509 & 18 & 1590 & 32 & 5 \\
\hline 30.1 & 101 & 316 & 3.12 & 50.5 & 0.000060 & 0.10 & 5.368 & 0.067 & 0.0771 & 0.0007 & 0.1861 & 0.0023 & 1.956 & 0.036 & 0.0762 & 0.0010 & 0.672 & 1100 & 13 & 1102 & 27 & 0 \\
\hline 31.1 & 21 & 39 & 1.85 & 5.5 & - & $<0.01$ & 6.073 & 0.148 & 0.0659 & 0.0022 & 0.1662 & 0.0042 & 1.693 & 0.142 & 0.0739 & 0.0059 & 0.301 & 991 & 23 & 1038 & 162 & 4 \\
\hline 32.1 & 291 & 569 & 1.95 & 48.3 & 0.001326 & 2.38 & 10.130 & 0.116 & 0.0760 & 0.0007 & 0.0967 & 0.0011 & 0.785 & 0.030 & 0.0588 & 0.0022 & 0.298 & 595 & 7 & 561 & 80 & -6 \\
\hline
\end{tabular}

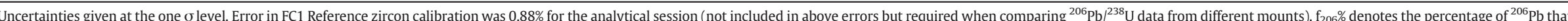
is common Pb. Correction for common $\mathrm{Pb}$ made using the measured ${ }^{204} \mathrm{~Pb} /{ }^{206} \mathrm{~Pb}$ ratio. For \% Disc, $0 \%$ denotes a concordant analysis. $\mathrm{Pb}^{*}$ is the proportion of Pb that is radiogenic 
concordance on the Tera and Wasserburg plot), the relative concentrations of $U$ and $T h$, the nature of the area analysed when examined post analysis, and the abundance of a particular age grouping. Zircon age data from the samples are presented in Tables 2-9, and are shown on combined histogram-relative probability plots in Figs. 2 and 3.

\section{Zircon age data and provenance implications}

\subsection{Fennoscandian Shield}

The age spectra from two samples of river sediment derived from the northern part of the Fennoscandian domain in Sweden (Byskeälven and Raneälven) are relatively simple. The spectrum from Byskeälven has a bimodal pattern, with a main peak at $1.80 \mathrm{Ga}$ and a subordinate peak at $1.87 \mathrm{Ga}$ (Fig. 2). By contrast, the Raneälven spectrum has a single main peak at $1.89 \mathrm{Ga}$, although there is a small secondary group comprising five zircons dated between c. 1.76 and $1.79 \mathrm{Ga}$ (Fig. 2). Both samples have a small number of younger Proterozoic zircons ranging down to c. $940 \mathrm{Ma}$, the Byskeälven sample containing more of these than the Raneälven sample. Neither sample contains any unambiguous Archaean or pre-1.95 Ga zircons, apart from grain 48.1 in the Raneälven sample, which has an apparent age of c. 3.5 Ga but is markedly discordant (Table 3 ).

The main peaks in the Raneälven and Byskeälven zircon age spectra can be directly matched with Svecokarelian phase 1 and Svecokarelian phase 2 . The group peaking at $1.89 \mathrm{Ga}$ in the Raneälven sample directly correlates with the age range of the Svecokarelian phase 1, and the small number of zircons between c. 1.76-1.79 Ma correlate with the end of Svecokarelian phase 2. The main peak in the Byskeälven sample $(1.80 \mathrm{Ga})$ corresponds to Svecokarelian phase 2, and the subordinate peak at c. $1.87 \mathrm{Ga}$ is close to the range of Svecokarelian phase 1 . The origin of the small number of younger grains is more speculative. Those between c. 1.70 and 1.54 Ga may represent erosional products of post-orogenic granites, which are found sporadically within the Svecofennian Domain (Gaal and Gorbatschev, 1987; Koistinen et al., 2001). The small number of younger zircons, especially those between c. $940 \mathrm{Ma}$ and $1130 \mathrm{Ma}$, presumably diagnose minor input from the Caledonian Nappe Domain further to the west, although neither river appears to drain this region at present.

The absence of proven Archaean or pre-1.95 Ga zircons in the two samples indicates that very little detritus was supplied by the metasedimentary units within the Fennoscandian Shield area. The metasediments from the Svecofennian Domain contain two main groups of zircon, Archaean (mostly 2.97-2.60 Ga) and early Proterozoic (2.12-1.88 Ga), the latter being the predominant group (Claesson et al., 1993; Skiöld and Rutland, 2006; Bergman et al., 2008). The absence of such zircons in the two samples is interpreted as reflecting the biased nature of the zircon population, since it implies that most of the zircons were derived from the granitoid rocks and comparatively few from the metasediments.

\subsection{Archaean}

Lainioälven almost exclusively drains the Archaean domain in northern Sweden (Fig. 1). The age spectrum (Fig. 2) has a bimodal pattern, with a well-defined peak at c. $1.80 \mathrm{Ga}$ and a broader group between 2.65 and $2.78 \mathrm{Ga}$. The latter group corresponds to the age of the granitoids, migmatites and grey gneisses that form the Archaean basement in the Lainoalven drainage basin, which are considered to have formed between 2.75-2.50 Ga (Koistinen et al., 2001). The $1.80 \mathrm{Ga}$ peak directly matches the main peak in the sample from Byskeälven, indicating that the main phase of metamorphic reworking of the Archaean took place during Svecokarelian phase 2. There is no evidence for zircons derived from the Lapponian volcanic succession, which has an upper age limit of c. $2.3 \mathrm{Ga}$ (Gaal and Gorbatschev, 1987). This probably reflects the composition of the Lapponian volcanic succession, which consists predominantly of mafic or ultramafic rocks, lithologies that are generally zircon-poor. There is also no evidence for derivation from Saamian basement rocks (3.1-2.9 Ga), consistent with regional data that suggest basement of this age is confined to Finland and the Kola Peninsula (Koistinen et al., 2001).

\subsection{Trans-Scandinavian igneous belt}

The zircon population from the Ljusnan river, which drains the Dalarna province of the Trans-Scandinavian Igneous Belt (Fig. 1) comprises a single main group peaking at 1.70 Ga (Fig. 2), corresponding precisely to TIB2 as defined by (Larson and Berglund, 1992). Curiously, although both TIB1 (which correlates with the late Svecofennian granitoid intrusion phase) and TIB2 events are recorded in the Dalarna province (Larson and Berglund, 1992), the river sample only identifies the younger of the two episodes. This either indicates significant regional differences in age of the granitoid rocks in the Dalarna province, or that TIB1 granitoids are volumetrically minor in the Ljusnan river catchment. There are scattered younger zircons in the Ljusnan river sample, ranging down to c. $0.80 \mathrm{Ga}$. These zircons probably represent minor supply from the Caledonian Nappe Domain, since the Ljusnan catchment extends into a region comprising the Lower/Middle Allochthon.

\subsection{Caledonian Nappe Domain}

The four zircon age spectra from modern river sediments derived from the Caledonian Nappe Domain are broadly similar, being composed almost exclusively of Proterozoic zircons between $\mathrm{c}$. $0.80 \mathrm{Ga}$ and c. $1.85 \mathrm{Ga}$ (Fig. 3). This compares well with the only other available data from metasediments of the Caledonian Nappe Domain, since the 15 detrital zircons analysed by Williams and Claesson (1987) from the Seve Nappes fall into the same age range. In addition, the Vefsna sample contains a significant number of Early Paleozoic zircons, which form c. 15\% (5 out of 32 grains) of the population, and a single late Neoproterozoic grain ( $595 \pm 7 \mathrm{Ma})$. The Saltelva and Vesfna samples also contain a small number of Archaean grains, two being present in the Saltelva sample and one in the Vefsna sample.

The four spectra share several common elements within the Proterozoic part of the spectra (Fig. 4). Vefsna, Saltelva and Vindelälven all show a major peak at c.1.79-1.80 Ga, an event that is poorly represented in the Ljungan sample. All samples have major group at c.1.64-1.67 Ga, together with younger zircons ranging down to c. $1.55 \mathrm{Ga}$. Saltelva, Vindelälven and Ljungan contain significant clusters of zircons between c. $1.45 \mathrm{Ma}$ and $1.50 \mathrm{Ga}$. There is relatively little representation between $1.40 \mathrm{Ga}$ and $1.20 \mathrm{Ga}$. All four samples have broadly bimodal patterns within the $0.90-1.22$ Ga range, with peaks between c. $1.15 \mathrm{Ga}$ and $1.22 \mathrm{Ga}$ and between c. $0.95 \mathrm{Ga}$ and $1.06 \mathrm{Ga}$. In addition, the Vefsna sample has minor peaks at c. $1.74 \mathrm{Ga}$ and c. $1.10 \mathrm{Ga}$ that are not represented in the other samples.

These peaks can be directly related to events in Baltica. The peak at c. 1.79-1.80 Ga group is the same age as the basement windows and allochthonous granites within the Caledonian Nappe Domain (Skår, 2002; Rehnström and Corfu, 2004), which correlate with Svecokarelian phase 2 granitoids and the oldest Trans-Scandinavian event, TIB1 (Gaal and Gorbatschev, 1987; Larson and Berglund, 1992; Koistinen et al., 2001). The minor peak at c. $1.74 \mathrm{Ga}$ in the Vefsna sample corresponds to the ages of granitoids in the Rombak-Sjangeli basement window (Rehnström and Corfu, 2004). The 1.64-1.67 Ga peak corresponds to the Gothian/Kongsbergian orogeny, which is the main crust-forming event in southwest Scandinavia and the Western Gneiss Region (Gaal and Gorbatschev, 1987; Gower et al., 1991; Tucker et al., 
Table 8

Summary of SHRIMP U-Pb zircon results for Vindelälven river sand

\begin{tabular}{|c|c|c|c|c|c|c|c|c|c|c|c|c|c|c|c|c|c|c|c|c|c|c|}
\hline \multirow[b]{2}{*}{$\begin{array}{l}\text { Grain. } \\
\text { spot }\end{array}$} & \multirow[b]{2}{*}{$\begin{array}{l}\mathrm{U} \\
(\mathrm{ppm})\end{array}$} & \multirow[b]{2}{*}{$\begin{array}{l}\text { Th } \\
(\mathrm{ppm})\end{array}$} & \multirow[b]{2}{*}{$\mathrm{Th} / \mathrm{U}$} & \multirow[b]{2}{*}{$\begin{array}{l}\mathrm{Pb}^{*} \\
(\mathrm{ppm})\end{array}$} & \multirow[b]{2}{*}{${ }^{204} \mathrm{~Pb} /{ }^{206} \mathrm{~Pb}$} & \multirow[b]{2}{*}{$f_{206} \%$} & \multicolumn{4}{|l|}{ Total ratios } & \multicolumn{6}{|c|}{ Radiogenic ratios } & \multirow[b]{2}{*}{$\mathrm{r}$} & \multicolumn{4}{|l|}{ Age (Ma) } & \multirow[b]{2}{*}{$\%$ Disc } \\
\hline & & & & & & & ${ }^{238} \mathrm{U} /{ }^{206} \mathrm{~Pb}$ & \pm & ${ }^{207} \mathrm{~Pb} /{ }^{206} \mathrm{~Pb}$ & \pm & ${ }^{206} \mathrm{~Pb} /{ }^{238} \mathrm{U}$ & \pm & ${ }^{207} \mathrm{~Pb} /{ }^{235} \mathrm{U}$ & \pm & ${ }^{207} \mathrm{~Pb} /{ }^{206} \mathrm{~Pb}$ & \pm & & ${ }^{206} \mathrm{~Pb} /{ }^{238} \mathrm{U}$ & \pm & ${ }^{207} \mathrm{~Pb} /{ }^{206} \mathrm{~Pb}$ & \pm & \\
\hline 1.1 & 139 & 93 & 0.67 & 34.2 & 0.000048 & 0.08 & 3.486 & 0.075 & 0.1003 & 0.0012 & 0.2866 & 0.0062 & 3.938 & 0.101 & 0.0996 & 0.0014 & 0.845 & 1625 & 31 & 1617 & 25 & 0 \\
\hline 2.1 & 83 & 51 & 0.61 & 12.5 & 0.000908 & 1.57 & 5.726 & 0.100 & 0.0805 & 0.0016 & 0.1723 & 0.0030 & 1.646 & 0.058 & 0.0693 & 0.0021 & 0.492 & 1025 & 19 & 907 & 64 & -13 \\
\hline 3.1 & 530 & 141 & 0.27 & 98.0 & 0.000010 & 0.02 & 4.647 & 0.054 & 0.0806 & 0.0006 & 0.2152 & 0.0025 & 2.388 & 0.034 & 0.0805 & 0.0007 & 0.808 & 1256 & 13 & 1209 & 17 & -4 \\
\hline 4.1 & 184 & 68 & 0.37 & 51.6 & - & $<0.01$ & 3.060 & 0.041 & 0.1115 & 0.0010 & 0.3272 & 0.0044 & 5.084 & 0.087 & 0.1127 & 0.0012 & 0.786 & 1825 & 21 & 1843 & 19 & 1 \\
\hline 5.1 & 64 & 31 & 0.48 & 8.6 & - & $<0.01$ & 6.421 & 0.121 & 0.0734 & 0.0018 & 0.1560 & 0.0030 & 1.612 & 0.062 & 0.0749 & 0.0025 & 0.496 & 935 & 16 & 1066 & 67 & 12 \\
\hline 6.1 & 29 & 27 & 0.91 & 4.7 & 0.000496 & 0.82 & 5.349 & 0.135 & 0.0936 & 0.0029 & 0.1854 & 0.0048 & 2.215 & 0.171 & 0.0867 & 0.0063 & 0.338 & 1097 & 26 & 1353 & 140 & 19 \\
\hline 7.1 & 459 & 207 & 0.45 & 123.9 & - & $<0.01$ & 3.183 & 0.037 & 0.1070 & 0.0006 & 0.3144 & 0.0037 & 4.665 & 0.061 & 0.1076 & 0.0006 & 0.901 & 1762 & 19 & 1760 & 10 & 0 \\
\hline 8.1 & 723 & 110 & 0.15 & 108.1 & 0.000015 & 0.03 & 5.743 & 0.065 & 0.0735 & 0.0005 & 0.1741 & 0.0020 & 1.759 & 0.024 & 0.0733 & 0.0005 & 0.848 & 1035 & 11 & 1021 & 14 & -1 \\
\hline 9.1 & 222 & 94 & 0.42 & 31.2 & 0.000488 & 0.79 & 6.110 & 0.089 & 0.1006 & 0.0012 & 0.1624 & 0.0024 & 2.101 & 0.072 & 0.0939 & 0.0029 & 0.430 & 970 & 13 & 1505 & 59 & 36 \\
\hline 10.1 & 276 & 67 & 0.24 & 74.6 & 0.000080 & 0.13 & 3.182 & 0.040 & 0.1107 & 0.0008 & 0.3138 & 0.0040 & 4.742 & 0.074 & 0.1096 & 0.0010 & 0.807 & 1760 & 19 & 1792 & 17 & 2 \\
\hline 11.1 & 618 & 203 & 0.33 & 164.0 & 0.000025 & 0.04 & 3.237 & 0.039 & 0.1100 & 0.0006 & 0.3088 & 0.0037 & 4.671 & 0.063 & 0.1097 & 0.0007 & 0.890 & 1735 & 18 & 1794 & 11 & 3 \\
\hline 12.1 & 199 & 128 & 0.65 & 46.4 & 0.000028 & 0.05 & 3.681 & 0.049 & 0.0988 & 0.0010 & 0.2715 & 0.0036 & 3.683 & 0.063 & 0.0984 & 0.0010 & 0.784 & 1548 & 18 & 1594 & 20 & 3 \\
\hline 13.1 & 180 & 72 & 0.40 & 29.0 & 0.000156 & 0.26 & 5.311 & 0.079 & 0.0794 & 0.0013 & 0.1878 & 0.0028 & 1.998 & 0.061 & 0.0772 & 0.0020 & 0.494 & 1109 & 15 & 1126 & 53 & 1 \\
\hline 14.1 & 531 & 116 & 0.22 & 115.3 & 0.000027 & 0.04 & 3.956 & 0.047 & 0.0999 & 0.0006 & 0.2527 & 0.0030 & 3.468 & 0.047 & 0.0995 & 0.0007 & 0.865 & 1452 & 15 & 1616 & 13 & 10 \\
\hline 15.1 & 417 & 239 & 0.57 & 82.7 & 0.000046 & 0.07 & 4.331 & 0.054 & 0.0917 & 0.0009 & 0.2307 & 0.0029 & 2.898 & 0.049 & 0.0911 & 0.0010 & 0.740 & 1338 & 15 & 1448 & 22 & 8 \\
\hline 16.1 & 298 & 204 & 0.68 & 72.8 & 0.000146 & 0.23 & 3.523 & 0.044 & 0.1032 & 0.0008 & 0.2832 & 0.0036 & 3.952 & 0.067 & 0.1012 & 0.0011 & 0.746 & 1608 & 18 & 1646 & 21 & 2 \\
\hline 17.1 & 679 & 347 & 0.51 & 96.4 & 0.000164 & 0.27 & 6.049 & 0.071 & 0.0887 & 0.0007 & 0.1649 & 0.0020 & 1.963 & 0.039 & 0.0864 & 0.0014 & 0.598 & 984 & 11 & 1347 & 31 & 27 \\
\hline 18.1 & 1191 & 421 & 0.35 & 261.7 & 0.000130 & 0.21 & 3.909 & 0.044 & 0.0928 & 0.0004 & 0.2553 & 0.0029 & 3.204 & 0.041 & 0.0910 & 0.0006 & 0.878 & 1466 & 15 & 1447 & 12 & -1 \\
\hline 19.1 & 505 & 248 & 0.49 & 124.8 & - & $<0.01$ & 3.476 & 0.041 & 0.1012 & 0.0006 & 0.2877 & 0.0034 & 4.016 & 0.054 & 0.1012 & 0.0006 & 0.880 & 1630 & 17 & 1647 & 12 & 1 \\
\hline 20.1 & 448 & 783 & 1.75 & 102.4 & 0.000050 & 0.08 & 3.762 & 0.045 & 0.0937 & 0.0007 & 0.2656 & 0.0032 & 3.406 & 0.051 & 0.0930 & 0.0008 & 0.801 & 1518 & 16 & 1488 & 17 & -2 \\
\hline 21.1 & 91 & 47 & 0.52 & 19.0 & 0.000072 & 0.12 & 4.105 & 0.124 & 0.0918 & 0.0018 & 0.2433 & 0.0074 & 3.047 & 0.113 & 0.0908 & 0.0020 & 0.813 & 1404 & 38 & 1443 & 41 & 3 \\
\hline 22.1 & 209 & 92 & 0.44 & 46.2 & 0.000375 & 0.59 & 3.877 & 0.052 & 0.1091 & 0.0011 & 0.2564 & 0.0035 & 3.675 & 0.083 & 0.1039 & 0.0019 & 0.592 & 1471 & 18 & 1696 & 34 & 13 \\
\hline 23.1 & 684 & 529 & 0.77 & 162.8 & 0.000002 & $<0.01$ & 3.611 & 0.042 & 0.1013 & 0.0006 & 0.2769 & 0.0032 & 3.868 & 0.051 & 0.1013 & 0.0006 & 0.883 & 1576 & 16 & 1648 & 11 & 4 \\
\hline 24.1 & 467 & 87 & 0.19 & 118.8 & 0.000006 & 0.01 & 3.378 & 0.041 & 0.1099 & 0.0007 & 0.2960 & 0.0036 & 4.483 & 0.063 & 0.1098 & 0.0008 & 0.864 & 1671 & 18 & 1797 & 13 & 7 \\
\hline 25.1 & 271 & 78 & 0.29 & 64.9 & 0.000105 & 0.17 & 3.591 & 0.047 & 0.1020 & 0.0009 & 0.2780 & 0.0037 & 3.855 & 0.065 & 0.1006 & 0.0011 & 0.775 & 1581 & 18 & 1635 & 20 & 3 \\
\hline 26.1 & 477 & 116 & 0.24 & 129.4 & 0.000022 & 0.03 & 3.167 & 0.037 & 0.1089 & 0.0007 & 0.3156 & 0.0037 & 4.726 & 0.064 & 0.1086 & 0.0007 & 0.868 & 1768 & 18 & 1776 & 12 & 0 \\
\hline 27.1 & 43 & 17 & 0.38 & 7.5 & 0.000649 & 1.11 & 4.980 & 0.117 & 0.0802 & 0.0027 & 0.2013 & 0.0047 & 2.285 & 0.092 & 0.0823 & 0.0027 & 0.586 & 1182 & 27 & 1253 & 64 & 6 \\
\hline 28.1 & 209 & 211 & 1.01 & 26.2 & 0.000178 & 0.31 & 6.837 & 0.097 & 0.0715 & 0.0011 & 0.1458 & 0.0021 & 1.386 & 0.037 & 0.0689 & 0.0016 & 0.532 & 877 & 12 & 896 & 47 & 2 \\
\hline 29.1 & 86 & 43 & 0.50 & 12.1 & - & $<0.01$ & 6.074 & 0.110 & 0.0757 & 0.0018 & 0.1653 & 0.0030 & 1.797 & 0.071 & 0.0789 & 0.0028 & 0.465 & 986 & 17 & 1169 & 69 & 16 \\
\hline
\end{tabular}




\begin{tabular}{|c|c|c|c|c|c|c|c|c|c|c|c|c|c|c|c|c|c|c|c|c|c|c|}
\hline 30.1 & 35 & 21 & 0.60 & 6.4 & - & $<0.01$ & 4.716 & 0.116 & 0.0930 & 0.0027 & 0.2104 & 0.0052 & 2.503 & 0.103 & 0.0863 & 0.0029 & 0.595 & 1231 & 30 & 1345 & 64 & 8 \\
\hline 31.1 & 108 & 82 & 0.76 & 26.5 & 0.000210 & 0.34 & 3.494 & 0.057 & 0.1002 & 0.0014 & 0.2875 & 0.0047 & 4.114 & 0.088 & 0.1038 & 0.0014 & 0.762 & 1629 & 26 & 1693 & 25 & 4 \\
\hline 32.1 & 80 & 64 & 0.80 & 11.3 & 0.000387 & 0.65 & 6.097 & 0.124 & 0.0834 & 0.0020 & 0.1629 & 0.0034 & 1.750 & 0.123 & 0.0779 & 0.0052 & 0.301 & 973 & 19 & 1144 & 133 & 15 \\
\hline 33.1 & 145 & 41 & 0.28 & 21.3 & 0.000094 & 0.16 & 5.825 & 0.083 & 0.0734 & 0.0011 & 0.1714 & 0.0025 & 1.703 & 0.063 & 0.0721 & 0.0024 & 0.395 & 1020 & 14 & 988 & 69 & -3 \\
\hline 34.1 & 247 & 91 & 0.37 & 56.7 & 0.000056 & 0.09 & 3.747 & 0.047 & 0.0920 & 0.0008 & 0.2667 & 0.0034 & 3.353 & 0.055 & 0.0912 & 0.0009 & 0.770 & 1524 & 17 & 1451 & 20 & -5 \\
\hline 34.2 & 189 & 81 & 0.43 & 48.2 & 0.000048 & 0.08 & 3.371 & 0.047 & 0.1035 & 0.0010 & 0.2965 & 0.0041 & 4.206 & 0.074 & 0.1029 & 0.0011 & 0.789 & 1674 & 21 & 1677 & 20 & 0 \\
\hline 35.2 & 106 & 31 & 0.29 & 23.6 & 0.000124 & 0.20 & 3.842 & 0.059 & 0.0927 & 0.0013 & 0.2599 & 0.0040 & 3.274 & 0.069 & 0.0914 & 0.0013 & 0.731 & 1489 & 21 & 1455 & 27 & -2 \\
\hline 36.1 & 117 & 5 & 0.04 & 21.9 & - & $<0.01$ & 4.577 & 0.084 & 0.0876 & 0.0014 & 0.2185 & 0.0040 & 2.639 & 0.064 & 0.0876 & 0.0014 & 0.759 & 1274 & 21 & 1374 & 30 & 7 \\
\hline 37.1 & 186 & 98 & 0.53 & 46.8 & 0.000120 & 0.19 & 3.420 & 0.045 & 0.1055 & 0.0009 & 0.2919 & 0.0039 & 4.181 & 0.090 & 0.1039 & 0.0018 & 0.617 & 1651 & 19 & 1695 & 31 & 3 \\
\hline 38.1 & 73 & 32 & 0.44 & 16.8 & 0.000115 & 0.19 & 3.720 & 0.063 & 0.0916 & 0.0014 & 0.2683 & 0.0046 & 3.330 & 0.077 & 0.0900 & 0.0014 & 0.730 & 1532 & 25 & 1426 & 30 & -7 \\
\hline 39.1 & 576 & 236 & 0.41 & 142.2 & 0.000018 & 0.03 & 3.481 & 0.040 & 0.1016 & 0.0005 & 0.2872 & 0.0033 & 4.014 & 0.052 & 0.1014 & 0.0006 & 0.885 & 1627 & 16 & 1650 & 11 & 1 \\
\hline 40.1 & 138 & 31 & 0.23 & 37.3 & 0.000090 & 0.14 & 3.188 & 0.048 & 0.1078 & 0.0011 & 0.3135 & 0.0048 & 4.643 & 0.085 & 0.1074 & 0.0011 & 0.825 & 1758 & 24 & 1756 & 19 & 0 \\
\hline 41.1 & 827 & 285 & 0.30 & 190.3 & 0.000028 & 0.04 & 3.733 & 0.041 & 0.0999 & 0.0004 & 0.2678 & 0.0029 & 3.674 & 0.044 & 0.0995 & 0.0005 & 0.921 & 1530 & 15 & 1615 & 9 & 5 \\
\hline 42.1 & 507 & 202 & 0.40 & 142.7 & 0.000076 & 0.12 & 3.052 & 0.035 & 0.1069 & 0.0006 & 0.3283 & 0.0038 & 4.905 & 0.063 & 0.1084 & 0.0006 & 0.904 & 1830 & 19 & 1772 & 10 & -3 \\
\hline 43.1 & 269 & 44 & 0.16 & 38.6 & 0.000066 & 0.11 & 5.984 & 0.086 & 0.0759 & 0.0042 & 0.1669 & 0.0024 & 1.726 & 0.103 & 0.0750 & 0.0043 & 0.242 & 995 & 13 & 1069 & 116 & 7 \\
\hline 44.1 & 128 & 86 & 0.67 & 33.4 & 0.000134 & 0.21 & 3.305 & 0.048 & 0.1034 & 0.0011 & 0.3038 & 0.0044 & 4.461 & 0.082 & 0.1065 & 0.0012 & 0.797 & 1710 & 24 & 1741 & 20 & 2 \\
\hline 45.1 & 47 & 18 & 0.38 & 8.2 & 0.000176 & 0.29 & 4.916 & 0.102 & 0.0819 & 0.0024 & 0.2028 & 0.0044 & 2.221 & 0.178 & 0.0794 & 0.0061 & 0.272 & 1190 & 24 & 1183 & 152 & -1 \\
\hline 46.1 & 369 & 246 & 0.67 & 86.2 & - & $<0.01$ & 3.679 & 0.044 & 0.0977 & 0.0007 & 0.2718 & 0.0033 & 3.671 & 0.051 & 0.0980 & 0.0007 & 0.861 & 1550 & 17 & 1586 & 13 & 2 \\
\hline 47.1 & 326 & 104 & 0.32 & 87.0 & 0.000004 & 0.01 & 3.225 & 0.039 & 0.1091 & 0.0007 & 0.3100 & 0.0038 & 4.660 & 0.065 & 0.1090 & 0.0008 & 0.863 & 1741 & 18 & 1783 & 13 & 2 \\
\hline 48.1 & 202 & 41 & 0.20 & 54.5 & 0.000107 & 0.17 & 3.191 & 0.042 & 0.1085 & 0.0009 & 0.3129 & 0.0042 & 4.617 & 0.079 & 0.1070 & 0.0012 & 0.775 & 1755 & 20 & 1750 & 20 & 0 \\
\hline 49.1 & 210 & 85 & 0.41 & 52.5 & 0.000055 & 0.09 & 3.434 & 0.050 & 0.1037 & 0.0010 & 0.2910 & 0.0042 & 4.129 & 0.077 & 0.1029 & 0.0012 & 0.778 & 1646 & 21 & 1678 & 22 & 2 \\
\hline 50.1 & 80 & 77 & 0.96 & 20.9 & 0.000040 & 0.06 & 3.274 & 0.055 & 0.1016 & 0.0014 & 0.3062 & 0.0051 & 4.374 & 0.095 & 0.1036 & 0.0014 & 0.775 & 1722 & 29 & 1690 & 25 & -2 \\
\hline 51.1 & 214 & 100 & 0.47 & 57.0 & - & $<0.01$ & 3.222 & 0.042 & 0.1106 & 0.0009 & 0.3104 & 0.0041 & 4.745 & 0.074 & 0.1109 & 0.0009 & 0.840 & 1743 & 20 & 1814 & 15 & 4 \\
\hline 52.1 & 611 & 157 & 0.26 & 159.9 & 0.000065 & 0.10 & 3.283 & 0.039 & 0.1100 & 0.0006 & 0.3043 & 0.0036 & 4.576 & 0.062 & 0.1091 & 0.0007 & 0.880 & 1713 & 18 & 1784 & 12 & 4 \\
\hline 53.1 & 32 & 12 & 0.38 & 8.5 & 0.000643 & 1.02 & 3.249 & 0.081 & 0.1092 & 0.0025 & 0.3066 & 0.0077 & 4.485 & 0.156 & 0.1061 & 0.0026 & 0.718 & 1724 & 40 & 1733 & 44 & 1 \\
\hline 54.1 & 493 & 203 & 0.41 & 118.7 & - & $<0.01$ & 3.570 & 0.042 & 0.1021 & 0.0006 & 0.2801 & 0.0033 & 3.943 & 0.052 & 0.1021 & 0.0006 & 0.893 & 1592 & 17 & 1662 & 11 & 4 \\
\hline 55.1 & 511 & 297 & 0.58 & 69.7 & 0.000034 & 0.06 & 6.300 & 0.074 & 0.0712 & 0.0006 & 0.1586 & 0.0019 & 1.548 & 0.023 & 0.0708 & 0.0006 & 0.791 & 949 & 10 & 951 & 19 & 0 \\
\hline 56.1 & 421 & 311 & 0.74 & 111.8 & 0.000058 & 0.09 & 3.236 & 0.040 & 0.1078 & 0.0013 & 0.3088 & 0.0038 & 4.556 & 0.079 & 0.1070 & 0.0013 & 0.715 & 1735 & 19 & 1749 & 22 & 1 \\
\hline 57.1 & 220 & 93 & 0.42 & 49.4 & 0.000115 & 0.19 & 3.817 & 0.050 & 0.0938 & 0.0009 & 0.2622 & 0.0034 & 3.418 & 0.055 & 0.0945 & 0.0009 & 0.810 & 1501 & 19 & 1519 & 18 & 1 \\
\hline 58.1 & 338 & 83 & 0.24 & 95.5 & 0.000033 & 0.05 & 3.044 & 0.039 & 0.1088 & 0.0007 & 0.3289 & 0.0042 & 4.972 & 0.071 & 0.1096 & 0.0007 & 0.897 & 1833 & 21 & 1793 & 12 & -2 \\
\hline 59.1 & 66 & 64 & 0.97 & 16.2 & 0.000222 & 0.35 & 3.477 & 0.063 & 0.1036 & 0.0017 & 0.2866 & 0.0052 & 3.973 & 0.128 & 0.1006 & 0.0027 & 0.563 & 1624 & 26 & 1634 & 50 & 1 \\
\hline 60.1 & 707 & 244 & 0.34 & 192.5 & 0.000043 & 0.07 & 3.158 & 0.036 & 0.1118 & 0.0005 & 0.3165 & 0.0036 & 4.855 & 0.061 & 0.1113 & 0.0006 & 0.910 & 1773 & 18 & 1820 & 9 & 3 \\
\hline
\end{tabular}

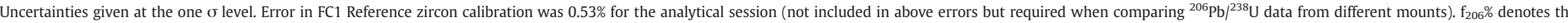
percentage of ${ }^{206} \mathrm{~Pb}$ that is common Pb. Correction for common $\mathrm{Pb}$ made using the measured $\left.{ }^{204} \mathrm{~Pb}\right|^{206} \mathrm{~Pb}$ ratio. For \% Disc, $0 \%$ denotes a concordant analysis. Pb* is the proportion of $\mathrm{Pb}$ that is radiogenic. 

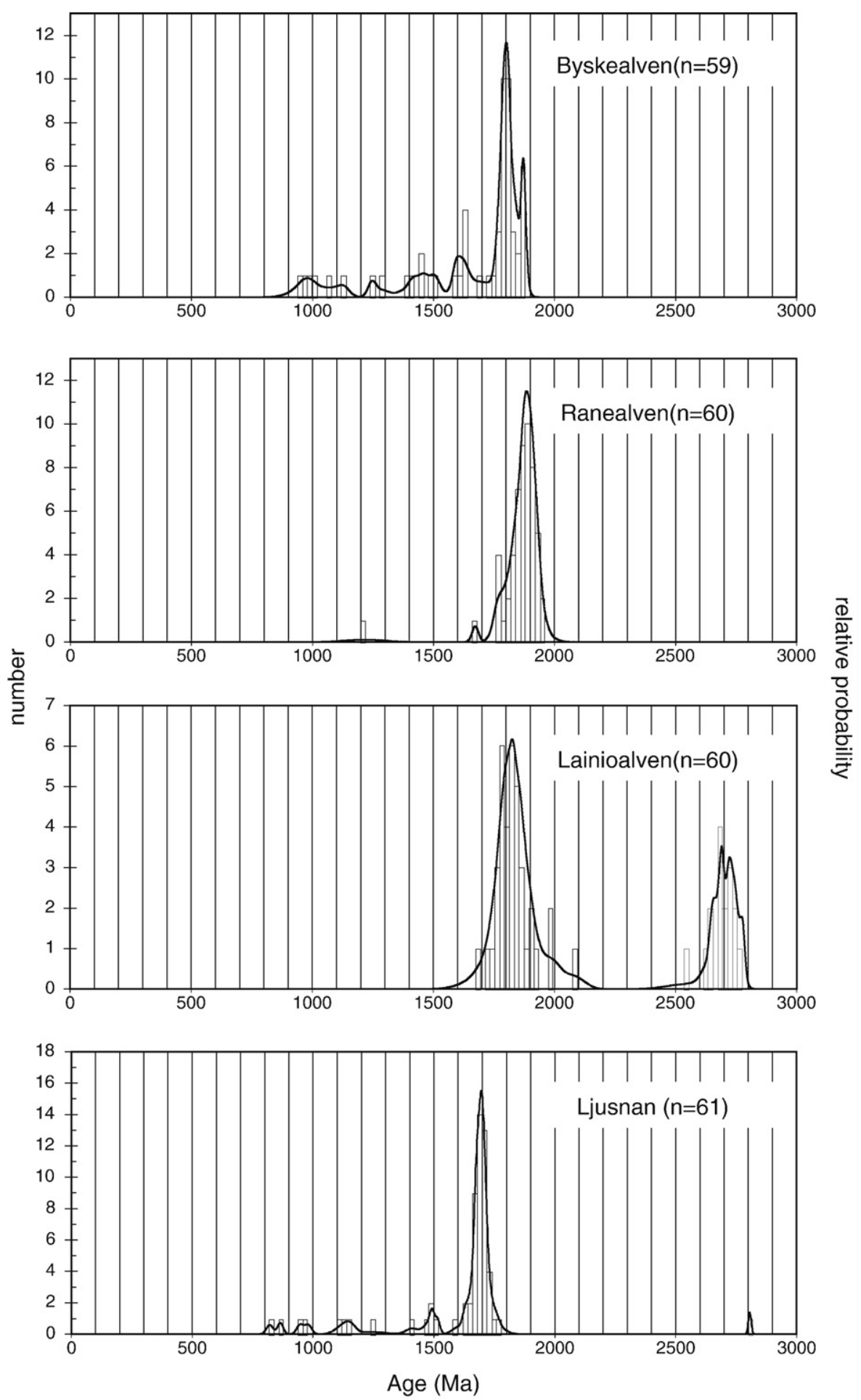

Fig. 2. Combined histogram-relative probability plots of zircon ages in rivers draining the Fennoscandian Shield and Trans-Scandinavian Igneous Belt.

1991; Skår and Pedersen, 2003). The 1.45-1.50 Ga peak corresponds to felsic magmatism in various parts of southern Scandinavia (Gower et al., 1991). The 1.15-1.22 Ga range is coincident with the onset of the Sveconorwegian orogeny, the effects of which continued until c. 0.90 Ga (Gower et al., 1991; Starmer, 1996).

The 1.79-1.80 Ga peak in the Vefsna, Saltelva and Vindelälven samples is found only in sediments from rivers with catchments that include, or are closely adjacent to, the basement windows. It is there- fore likely that this peak reflects first-cycle derivation from the basement window exposures, rather than recycling from Caledonian Nappe metasediments. This view is given further credence by the poor representation of the 1.79-1.80 Ga event in the Ljungan sample, whose catchment does not include basement windows.

Although the zircon age spectra in the rivers draining the Caledonian Nappe Domain can be reconciled with ultimate derivation from Baltica, Laurentia cannot be ruled out, since Laurentian and Baltican zircon age signatures are closely comparable (Cawood et al., 2007). Hence, some 

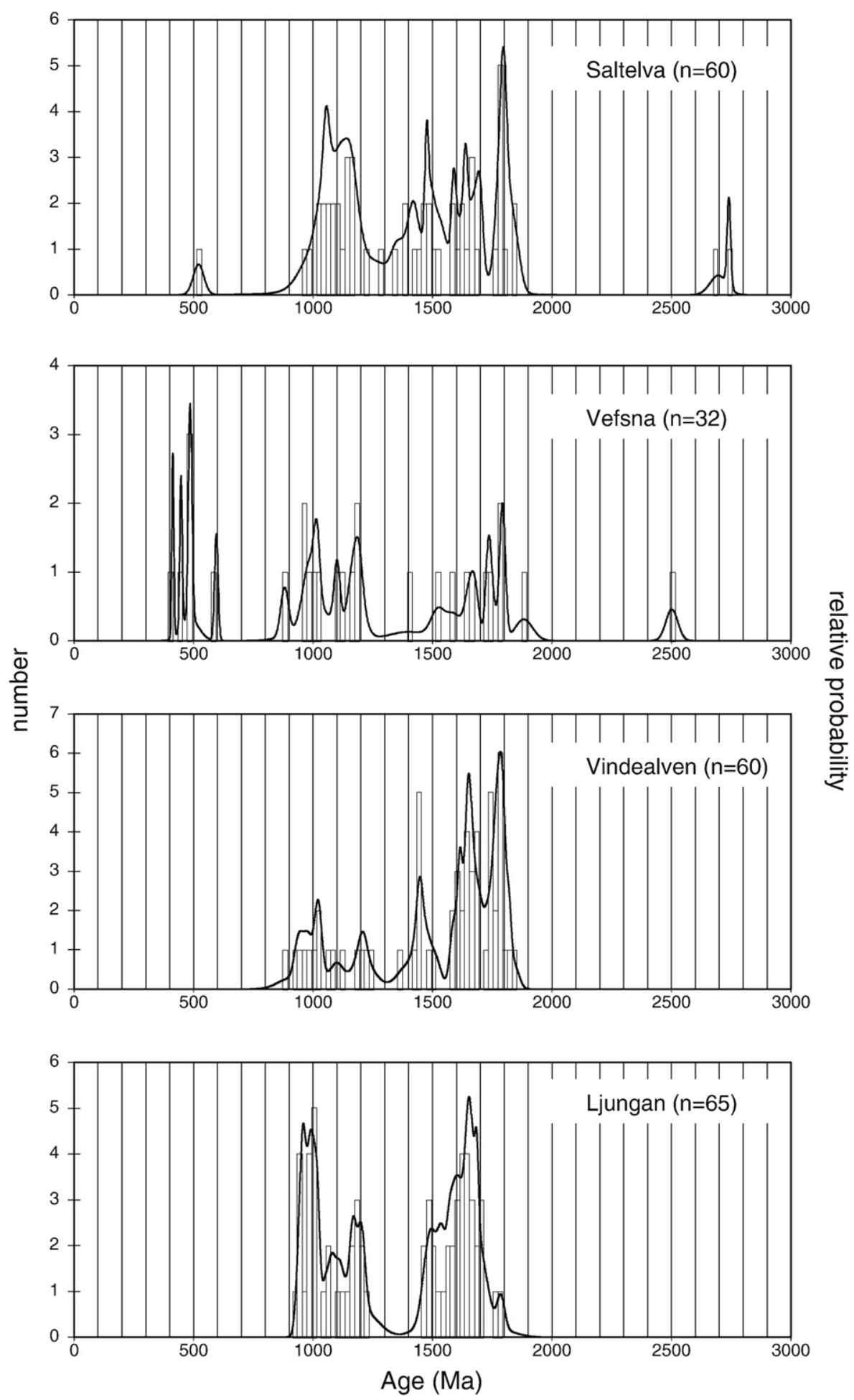

Fig. 3. Combined histogram-relative probability plots of zircon ages in rivers draining the Caledonian Nappe Domain.

of the zircons in the Saltelva and Vesfna samples, which were derived from the Upper and Uppermost Allochthon, could be ultimately of Laurentian origin, since the metasediments of the Uppermost Allochthon are believed to have had a Laurentian ancestry (Roberts, 2003; Kirkland et al., 2007).

The relatively large number of Early Paleozoic zircons in the Vefsna sample (Fig. 3, Table 7) reflects the widespread occurrence of Caledonian granitoids in the catchment of this river, represented by diorite-granite plutons within the Western Helgeland nappes. These granitoids have been dated as 443-447 Ma and 469-477 Ma (Bingen et al., 2002: Yoshinobu et al., 2002). The former of these two groups is represented by grain $28.1(447 \pm 5 \mathrm{Ma})$, and the latter corresponds reasonably closely with grains 20.1, 22.1 and 26.1 (486 $\pm 7,495 \pm 7$ and $486 \pm 8$ Ma respectively). Derivation from high-grade Caledonian metamorphic rocks can be ruled out because the zircons have relatively high Th/U ratios (Table 7). The late Neoproterozoic grain in the Vefsna 

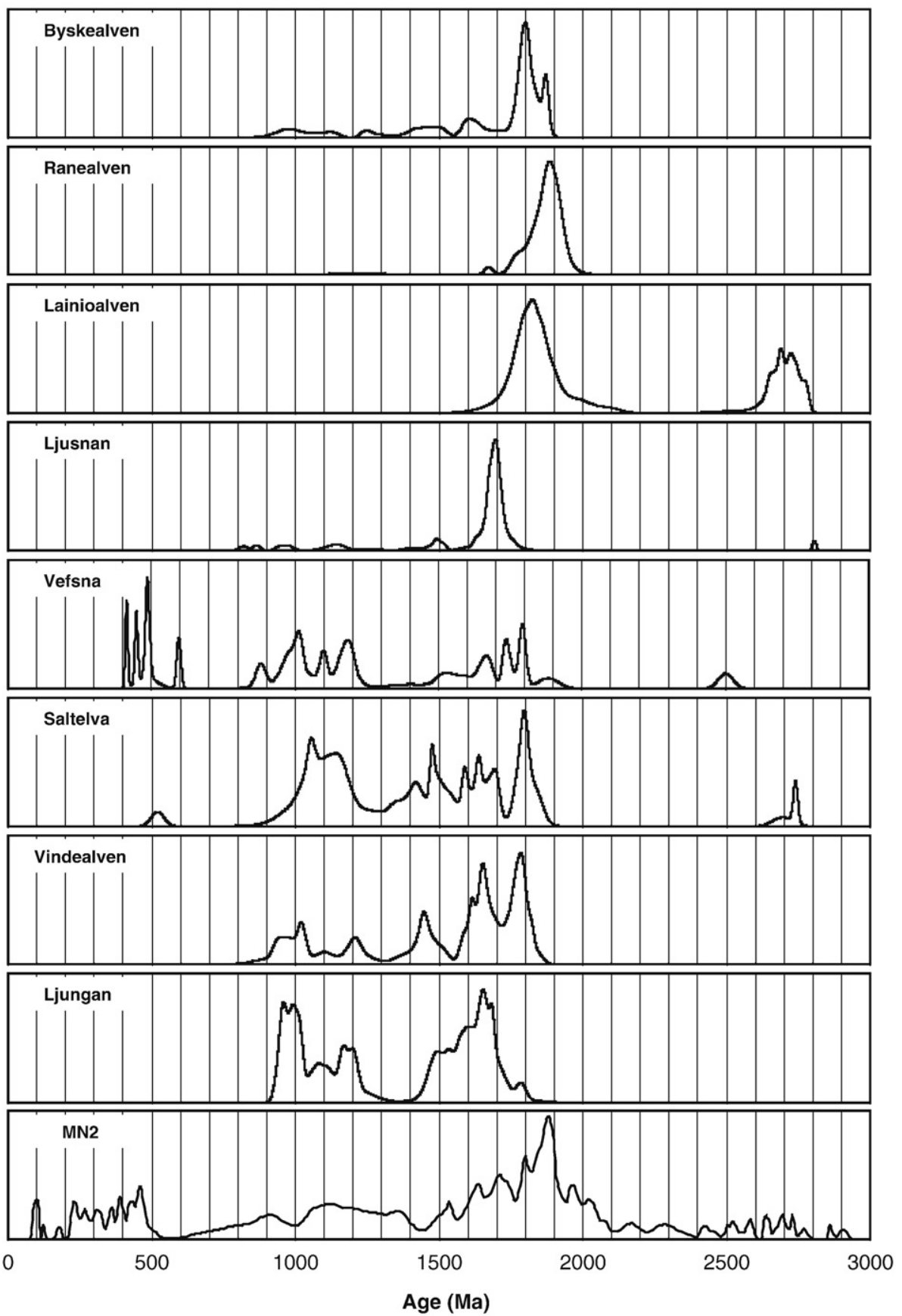

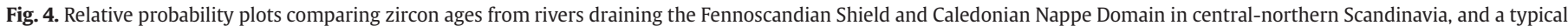
MN2 sandstone (Well 6607/5-2, $4172.0 \mathrm{~m}$ depth) from the Norwegian Sea.

sample corresponds to the major igneous pulse related to the opening of Iapetus. This is well represented in Baltica by the presence of mafic dykes dated as $616 \pm 3 \mathrm{Ma}$ (Bingen et al., 1998), although these are likely to be zircon-poor. There was also igneous activity of this age on the Laurentian margin (Cawood et al., 2001), in this case including felsic bodies that are more likely to supply zircons.

\subsection{Comparison with the Cretaceous of the Norwegian Sea}

A combined heavy mineral, mineral chemical and zircon age study of Cretaceous-Paleocene sandstones of the Norwegian Sea (Morton et al., 2005a,b) identified three different sand types in the basin, termed MN1, MN2 and MN3. Sand types MN1 and MN3 can be confidently tied back to source areas on the Norwegian margin, MN1 being derived from the northern Nordland area and MN3 to western Norway, including the Western Gneiss Region (Fig. 5). MN2 sandstones, by contrast, have a complex provenance involving Archaean and Early Proterozoic basement, Proterozoic metasedimentary rocks, Caledonian granites and Permo-Triassic and Early Cretaceous felsic igneous rocks, all of which occur, or can be inferred to occur, on the East Greenland margin. MN2 sandstones have therefore been interpreted as having an East Greenland source (Fonneland et al., 2004; Morton et al., 2005a,b). However, a source from the Scandinavian margin cannot be entirely ruled out because of the scarcity of data on detrital zircons in the metasediments of the Caledonian Nappe Domain. These metasediments could include 
Archaean and Early Proterozoic zircons absent from Norwegian basement rocks, by analogy with Dalradian metasedimentary rocks in Scotland. The Dalradian succession is broadly equivalent to the metasediments of the Caledonian Nappe Domain and is known to contain a significant proportion of Archaean zircons (Cawood et al., 2003). Another possibility is that the Archaean and Early Proterozoic zircons in the MN2 sandstones were supplied from the Fennoscandian Shield to the east of the Caledonides.

A comparison of the MN2 zircon age spectrum with those found in Scandinavian river sediments (Fig. 4) shows that the MN2 spectrum could not have been generated by recycling of Caledonian Nappe metasediments similar to those sampled by the modern rivers. The Caledonian Nappe metasediments are scarce in zircons older than c. $1.8 \mathrm{Ga}$, whereas such zircons are abundant in MN2 sandstones. The pre-1.8 Ga population in MN2 sandstones includes both Early Proterozoic and Archaean zircons. Only three of the 217 zircons analysed from the rivers draining the Caledonian Nappe Domain are Archaean, in contrast to the MN2 spectrum (Fig. 5), where c. 10\% of the zircon population is Archaean. Furthermore, the main peak in MN2 is at c. $1.90 \mathrm{Ga}$, significantly older than the oldest Proterozoic zircons in the rivers draining the Caledonian Nappe Domain.

The MN2 spectrum can also not be reconciled with a proposed origin through combined recyling of the Caledonian Nappe metasediments plus first-cycle derivation from the Fennoscandian Shield. It would be possible to supply the requisite number of Archaean zircons through this mechanism, providing derivation was from the Archaean realm of the Fennoscandian Shield in the northern part of Sweden, which supplied the sediment in Lainioälven, as discussed above. However, the Archaean in this region is heavily intruded by Late Svecofennian granitoids dated at c. $1.80 \mathrm{Ga}$, as demonstrated by the Lainioälven spectrum (Fig. 4). Since the MN2 spectrum lacks a significant peak at c. $1.80 \mathrm{Ga}$, derivation from this part of the Fennoscandian Shield can be discounted. Furthermore, the MN2 spectrum contains a small but significant number of pre-1.95 Ga zircons, which are almost entirely absent in sediments derived from either the Fennoscandian Shield or the Caledonian Nappe Domain. Involvement of sediment derived from the Fennoscandian Shield can therefore be ruled out. The c. 1.90 Ga peak that is a characteristic feature of the MN2 spectrum is also found in Devonian and Cretaceous sandstones of northern East Greenland, and is evidently of local origin (Knudsen et al., 2001; Morton et al., 2005b). Derivation from LofotenVesterålen can be ruled out, since although there are exposures of Archaean rocks dated as c. 2600 Ma by Jacobsen and Wasserburg (1978), these are not spatially associated with a potential source of abundant pre- 1800 Ma Proterozoic zircons, the Early Proterozoic of Lofoten-Vesterålen and the adjacent basement windows in the Nordland region being dated as c. 1770-1800 Ma (Skår, 2002; Corfu, 2004). There can therefore be little doubt that MN2 sandstones were not derived from Scandinavia, leaving East Greenland as the only viable source area.

\section{Conclusions}

The zircon age spectra in river sediments derived from the Caledonian Nappe Domain are fundamentally different to those derived from the Fennoscandian Shield. Whereas the Fennoscandian Shield spectra are comparatively simple, with one or two sharp and well-defined peaks, the Caledonian Nappe Domain spectra are much more diverse, with a wider range of ages and multiple peaks being present. The greater diversity largely reflects the nature of the sediment source, since most of the zircons from the Caledonian domain have been derived from metasedimentary rocks, which were themselves derived from the variety of precursor sources available at the time. For the most part, these precursor sources lay on the Baltican margin, although the Uppermost Allochthon may include sediment derived from Laurentia (Roberts, 2003). By contrast, the Fennoscan- dian Shield zircons were largely derived from granitoid rocks formed during specific crust-forming events. Thus, the Fennoscandian Shield spectra are typical of first-cycle, basement-derived detritus, whereas the Caledonian Nappe Domain spectra are typical of a multicyclic source.

The results from the river sediment study highlight a problem concerning the definition of polycyclic sediment. In lithological and mineralogical terms, the sediments derived from the Caledonian Nappe Domain would be classified as first-cycle, being predominantly sourced from metasedimentary rocks of varying metamorphic grades. However, the zircon population is predominantly recycled, the range of ages largely reflecting the variety of precursor sources that supplied the Caledonian metasedimentary successions. Integration of petrographic or mineralogical data with detrital zircon age data should clarify whether sediments are firstcycle or polycyclic.

Zircon age data from river sediment draining the Fennoscandian Shield faithfully reflect the ages of major phases of granitoid intrusion in these areas. The main peaks recognised in the Fennoscandian river sediments are (i) between 2.65 and $2.78 \mathrm{Ga}$, which corresponds to the age of the granitoids, migmatites and grey gneisses that form the Archaean basement of northern Sweden: (ii) $1.89 \mathrm{Ga}$ and $1.80 \mathrm{Ga}$, which represent Svecokarelian phases 1 and 2, and; (iii) $1.70 \mathrm{Ga}$, corresponding to the second phase of granitoid intrusion within the Trans-Scandinavian Igneous Belt belt (TIB2). The Fennoscandian zircon spectra show virtually no representation of Lapponian volcanics or Early Proterozoic metasediments, despite the presence of both lithologies within the drainage basins of the rivers concerned. The lack of Lapponian-age zircons is unsurprising given the mafic/ ultramafic nature of the volcanic succession, but the scarcity of zircons reworked from the metasediments is more enigmatic. The biased nature of the zircon populations is most likely to reflect a difference in relative abundance of zircons in the granitoids compared with the metasediments.

The main spectra in the river sediments derived from the Caledonian Nappe Domain also reflect major crust-forming events in Baltica, notably the Gothian/Kongsbergian and Sveconorwegian orogenies, plus intervening phases of anorogenic granitoid intrusion. Three of the four zircon populations also contain a peak at c. $1.80 \mathrm{Ga}$, the age of basement windows and allochthonous granites within the Caledonian Nappe Domain. These zircons are interpreted as being of first-cycle origin, rather than recycled from the Caledonian metasediments, since the catchments of the rivers containing these zircons include, or are closely adjacent to, the basement windows. One sample contains a high proportion of Early Paleozoic zircons derived from Caledonian granites, which are widespread in the catchment of this river. It is not possible to rule out ultimate derivation from Laurentia, since the zircon signature of Laurentian and Baltican detritus contain many common elements (Cawood et al., 2007), and the catchments of two of the rivers (Saltelva and Vefsna) include Laurentian-derived metasediments in the Uppermost Allochthon.

Comparison between the zircons in the Scandinavian rivers and Cretaceous sandstones in the Norwegian Sea demonstrate that recycling of Caledonian metasediments cannot account for the presence of Early Proterozoic and Archaean zircons in the MN2 group, as defined by Morton et al. (2005a). It is also difficult to reconcile the MN2 spectra with a combined provenance involving recycling of Caledonian metasediments and first-cycle sourcing from the Fennoscandian Shield. The most likely source of this sand type is therefore East Greenland, supporting previous interpretations of provenance data (Fonneland et al., 2004; Morton et al., 2005a,b).

\section{Acknowledgements}

We are grateful to Sarah Poynter, who collected the samples described in this paper, to Tasmin Coleman, who drafted some of the 
Table 9

Summary of SHRIMP U-Pb zircon results for Ljungan river sand

\begin{tabular}{|c|c|c|c|c|c|c|c|c|c|c|c|c|c|c|c|c|c|c|c|c|c|c|}
\hline \multirow[b]{2}{*}{$\begin{array}{l}\text { Grain. } \\
\text { spot }\end{array}$} & \multirow[b]{2}{*}{$\begin{array}{l}\mathrm{U} \\
(\mathrm{ppm})\end{array}$} & \multirow[b]{2}{*}{$\begin{array}{l}\text { Th } \\
(\mathrm{ppm})\end{array}$} & \multirow[b]{2}{*}{$\mathrm{Th} / \mathrm{U}$} & \multirow[b]{2}{*}{$\begin{array}{l}\mathrm{Pb}^{*} \\
(\mathrm{ppm})\end{array}$} & \multirow[b]{2}{*}{${ }^{204} \mathrm{~Pb} /{ }^{206} \mathrm{~Pb}$} & \multirow[b]{2}{*}{$\mathrm{f}_{206} \%$} & \multicolumn{4}{|l|}{ Total ratios } & \multicolumn{6}{|c|}{ Radiogenic ratios } & \multirow[b]{2}{*}{$\mathrm{r}$} & \multicolumn{4}{|l|}{ Age (Ma) } & \multirow[b]{2}{*}{ \% Disc } \\
\hline & & & & & & & ${ }^{238} \mathrm{U} /{ }^{206} \mathrm{~Pb}$ & \pm & ${ }^{207} \mathrm{~Pb} /{ }^{206} \mathrm{~Pb}$ & \pm & ${ }^{206} \mathrm{~Pb} /{ }^{238} \mathrm{U}$ & \pm & ${ }^{207} \mathrm{~Pb} /\left.\right|^{235} \mathrm{U}$ & \pm & ${ }^{207} \mathrm{~Pb} /{ }^{206} \mathrm{~Pb}$ & \pm & & ${ }^{206} \mathrm{~Pb} /{ }^{238} \mathrm{U}$ & \pm & ${ }^{207} \mathrm{~Pb} /{ }^{206} \mathrm{~Pb}$ & \pm & \\
\hline 1.1 & 260 & 70 & 0.27 & 46.0 & - & $<0.01$ & 4.859 & 0.059 & 0.0781 & 0.0008 & 0.2062 & 0.0026 & 2.263 & 0.051 & 0.0796 & 0.0012 & 0.793 & 1208 & 14 & 1187 & 29 & -2 \\
\hline 2.1 & 117 & 80 & 0.69 & 26.4 & - & $<0.01$ & 3.799 & 0.050 & 0.0924 & 0.0011 & 0.2632 & 0.0035 & 3.355 & 0.060 & 0.2632 & 0.0035 & 0.733 & 1506 & 18 & 1476 & 23 & -2 \\
\hline 3.1 & 55 & 20 & 0.37 & 9.5 & 0.000093 & 0.16 & 4.947 & 0.080 & 0.0788 & 0.0016 & 0.2020 & 0.0035 & 2.177 & 0.095 & 0.0782 & 0.0027 & 0.681 & 1186 & 19 & 1151 & 68 & -3 \\
\hline 4.1 & 50 & 55 & 1.10 & 9.5 & - & $<0.01$ & 4.492 & 0.073 & 0.0800 & 0.0016 & 0.2231 & 0.0036 & 2.512 & 0.080 & 0.2231 & 0.0036 & 0.514 & 1298 & 19 & 1238 & 53 & -5 \\
\hline 5.1 & 256 & 80 & 0.31 & 35.1 & 0.000062 & 0.11 & 6.272 & 0.073 & 0.0712 & 0.0008 & 0.1595 & 0.0019 & 1.571 & 0.040 & 0.0714 & 0.0013 & 0.759 & 954 & 11 & 970 & 37 & 2 \\
\hline 6.1 & 114 & 94 & 0.83 & 25.4 & 0.000100 & 0.16 & 3.853 & 0.050 & 0.0939 & 0.0011 & 0.2591 & 0.0033 & 3.304 & 0.064 & 0.2591 & 0.0033 & 0.660 & 1485 & 17 & 1477 & 28 & -1 \\
\hline 7.1 & 306 & 137 & 0.45 & 74.2 & 0.000011 & 0.02 & 3.547 & 0.041 & 0.0972 & 0.0007 & 0.2819 & 0.0033 & 3.774 & 0.051 & 0.2819 & 0.0033 & 0.858 & 1601 & 16 & 1569 & 13 & -2 \\
\hline 8.1 & 64 & 40 & 0.63 & 16.5 & - & $<0.01$ & 3.325 & 0.049 & 0.1043 & 0.0015 & 0.3008 & 0.0044 & 4.325 & 0.087 & 0.3008 & 0.0044 & 0.722 & 1695 & 22 & 1702 & 26 & 0 \\
\hline 9.1 & 213 & 135 & 0.64 & 55.1 & 0.000040 & 0.06 & 3.316 & 0.039 & 0.1032 & 0.0008 & 0.3014 & 0.0035 & 4.268 & 0.061 & 0.3014 & 0.0035 & 0.813 & 1698 & 17 & 1673 & 15 & -1 \\
\hline 10.1 & 89 & 33 & 0.37 & 20.5 & - & $<0.01$ & 3.745 & 0.051 & 0.0975 & 0.0013 & 0.2670 & 0.0037 & 3.590 & 0.068 & 0.2670 & 0.0037 & 0.727 & 1526 & 19 & 1577 & 24 & 3 \\
\hline 11.1 & 206 & 70 & 0.34 & 35.0 & - & $<0.01$ & 5.050 & 0.061 & 0.0787 & 0.0009 & 0.1980 & 0.0024 & 2.150 & 0.036 & 0.1980 & 0.0024 & 0.722 & 1165 & 13 & 1166 & 23 & 0 \\
\hline 12.1 & 592 & 191 & 0.32 & 81.4 & 0.000012 & 0.02 & 6.246 & 0.069 & 0.0714 & 0.0005 & 0.1601 & 0.0018 & 1.571 & 0.021 & 0.1601 & 0.0018 & 0.836 & 957 & 10 & 963 & 15 & 1 \\
\hline 13.1 & 126 & 35 & 0.28 & 19.7 & 0.000040 & 0.07 & 5.497 & 0.072 & 0.0721 & 0.0011 & 0.1821 & 0.0025 & 1.831 & 0.055 & 0.0729 & 0.0017 & 0.701 & 1078 & 14 & 1012 & 46 & -7 \\
\hline 14.1 & 204 & 100 & 0.49 & 46.9 & - & $<0.01$ & 3.729 & 0.044 & 0.0949 & 0.0008 & 0.2682 & 0.0032 & 3.513 & 0.051 & 0.2682 & 0.0032 & 0.806 & 1532 & 16 & 1528 & 16 & 0 \\
\hline 15.1 & 291 & 155 & 0.53 & 80.5 & 0.000109 & 0.17 & 3.108 & 0.035 & 0.1030 & 0.0007 & 0.3212 & 0.0036 & 4.493 & 0.065 & 0.3212 & 0.0036 & 0.786 & 1795 & 18 & 1651 & 17 & -9 \\
\hline 16.1 & 113 & 39 & 0.34 & 19.4 & 0.000081 & 0.14 & 5.028 & 0.068 & 0.0781 & 0.0011 & 0.1988 & 0.0028 & 2.136 & 0.069 & 0.0779 & 0.0019 & 0.723 & 1169 & 15 & 1145 & 48 & -2 \\
\hline 17.1 & 365 & 191 & 0.52 & 90.8 & 0.000028 & 0.05 & 3.453 & 0.038 & 0.1020 & 0.0006 & 0.2895 & 0.0032 & 4.054 & 0.053 & 0.2895 & 0.0032 & 0.859 & 1639 & 16 & 1653 & 12 & 1 \\
\hline 18.1 & 47 & 34 & 0.73 & 7.3 & - & $<0.01$ & 5.503 & 0.097 & 0.0758 & 0.0019 & 0.1817 & 0.0032 & 1.899 & 0.058 & 0.1817 & 0.0032 & 0.577 & 1076 & 17 & 1090 & 50 & 1 \\
\hline 19.1 & 116 & 44 & 0.38 & 16.5 & - & $<0.01$ & 6.062 & 0.083 & 0.0709 & 0.0012 & 0.1650 & 0.0023 & 1.613 & 0.036 & 0.1650 & 0.0023 & 0.623 & 984 & 13 & 955 & 35 & -3 \\
\hline 20.1 & 306 & 108 & 0.35 & 74.5 & 0.000064 & 0.10 & 3.530 & 0.042 & 0.1034 & 0.0008 & 0.2830 & 0.0033 & 3.998 & 0.059 & 0.2830 & 0.0033 & 0.805 & 1606 & 17 & 1669 & 16 & 4 \\
\hline 21.1 & 114 & 31 & 0.28 & 17.3 & - & $<0.01$ & 5.655 & 0.078 & 0.0727 & 0.0012 & 0.1768 & 0.0025 & 1.773 & 0.038 & 0.1768 & 0.0025 & 0.640 & 1050 & 13 & 1006 & 34 & -4 \\
\hline 22.1 & 146 & 99 & 0.68 & 38.0 & 0.000048 & 0.08 & 3.292 & 0.041 & 0.1022 & 0.0010 & 0.3039 & 0.0042 & 4.293 & 0.135 & 0.1025 & 0.0022 & 0.818 & 1711 & 21 & 1669 & 40 & -2 \\
\hline 23.1 & 83 & 30 & 0.36 & 11.2 & 0.000079 & 0.14 & 6.321 & 0.096 & 0.0701 & 0.0015 & 0.1583 & 0.0026 & 1.537 & 0.068 & 0.0704 & 0.0025 & 0.660 & 947 & 14 & 941 & 73 & -1 \\
\hline 24.1 & 65 & 44 & 0.68 & 14.7 & 0.000117 & 0.19 & 3.830 & 0.059 & 0.0922 & 0.0015 & 0.2609 & 0.0045 & 3.299 & 0.157 & 0.0917 & 0.0033 & 0.758 & 1495 & 23 & 1461 & 69 & -2 \\
\hline 25.1 & 1268 & 138 & 0.11 & 324.8 & 0.000013 & 0.02 & 3.354 & 0.035 & 0.1026 & 0.0004 & 0.2985 & 0.0031 & 4.253 & 0.052 & 0.1033 & 0.0004 & 0.947 & 1684 & 16 & 1685 & 8 & 0 \\
\hline 26.1 & 20 & 9 & 0.44 & 3.7 & - & $<0.01$ & 4.672 & 0.111 & 0.0794 & 0.0027 & 0.2140 & 0.0051 & 2.343 & 0.097 & 0.2140 & 0.0051 & 0.571 & 1250 & 27 & 1182 & 67 & -6 \\
\hline 27.1 & 323 & 130 & 0.40 & 72.7 & - & $<0.01$ & 3.817 & 0.043 & 0.0956 & 0.0007 & 0.2620 & 0.0030 & 3.454 & 0.046 & 0.2620 & 0.0030 & 0.846 & 1500 & 15 & 1540 & 13 & 3 \\
\hline 28.1 & 207 & 41 & 0.20 & 30.4 & - & $<0.01$ & 5.853 & 0.071 & 0.0736 & 0.0009 & 0.1709 & 0.0021 & 1.733 & 0.030 & 0.1709 & 0.0021 & 0.699 & 1017 & 11 & 1030 & 25 & 1 \\
\hline 29.1 & 88 & 0 & 0.00 & 14.3 & - & $<0.01$ & 5.270 & 0.076 & 0.0760 & 0.0013 & 0.1898 & 0.0027 & 1.989 & 0.045 & 0.1898 & 0.0027 & 0.633 & 1120 & 15 & 1096 & 35 & -2 \\
\hline 30.1 & 620 & 287 & 0.46 & 86.5 & - & $<0.01$ & 6.158 & 0.067 & 0.0708 & 0.0005 & 0.1624 & 0.0018 & 1.585 & 0.021 & 0.1624 & 0.0018 & 0.819 & 970 & 10 & 951 & 16 & -2 \\
\hline 31.1 & 386 & 291 & 0.75 & 88.8 & 0.001064 & 1.69 & 3.738 & 0.041 & 0.1145 & 0.0008 & 0.2630 & 0.0030 & 3.622 & 0.071 & 0.2630 & 0.0030 & 0.574 & 1505 & 15 & 1622 & 30 & 7 \\
\hline
\end{tabular}




\begin{tabular}{|c|c|c|c|c|c|c|c|c|c|c|c|c|c|c|c|c|c|c|c|c|c|c|}
\hline 32.1 & 134 & 209 & 1.56 & 32.3 & 0.000025 & 0.04 & 3.558 & 0.045 & 0.1009 & 0.0010 & 0.2809 & 0.0036 & 3.896 & 0.064 & 0.2809 & 0.0036 & 0.772 & 1596 & 18 & 1635 & 20 & 2 \\
\hline 33.1 & 171 & 119 & 0.70 & 43.0 & 0.000030 & 0.05 & 3.410 & 0.042 & 0.0981 & 0.0009 & 0.2937 & 0.0040 & 4.025 & 0.127 & 0.0994 & 0.0022 & 0.820 & 1660 & 20 & 1613 & 41 & -3 \\
\hline 34.1 & 41 & 30 & 0.72 & 11.4 & 0.000291 & 0.46 & 3.075 & 0.058 & 0.1110 & 0.0019 & 0.3243 & 0.0069 & 4.856 & 0.252 & 0.1086 & 0.0041 & 0.791 & 1811 & 33 & 1776 & 68 & -2 \\
\hline 35.1 & 278 & 89 & 0.32 & 40.0 & - & $<0.01$ & 5.973 & 0.070 & 0.0727 & 0.0008 & 0.1674 & 0.0019 & 1.678 & 0.026 & 0.1674 & 0.0019 & 0.738 & 998 & 11 & 1006 & 22 & 1 \\
\hline 36.1 & 71 & 65 & 0.91 & 18.8 & 0.000320 & 0.51 & 3.259 & 0.048 & 0.1057 & 0.0015 & 0.3053 & 0.0046 & 4.266 & 0.126 & 0.3053 & 0.0046 & 0.504 & 1718 & 23 & 1649 & 47 & -4 \\
\hline 37.1 & 100 & 40 & 0.40 & 13.4 & 0.000086 & 0.15 & 6.370 & 0.091 & 0.0708 & 0.0013 & 0.1568 & 0.0023 & 1.504 & 0.040 & 0.1568 & 0.0023 & 0.540 & 939 & 13 & 916 & 46 & -3 \\
\hline 38.1 & 171 & 119 & 0.70 & 43.6 & 0.000034 & 0.05 & 3.371 & 0.041 & 0.0994 & 0.0013 & 0.2964 & 0.0036 & 4.042 & 0.074 & 0.2964 & 0.0036 & 0.673 & 1674 & 18 & 1603 & 25 & -4 \\
\hline 39.1 & 36 & 50 & 1.41 & 9.2 & 0.000045 & 0.07 & 3.304 & 0.060 & 0.1047 & 0.0020 & 0.3024 & 0.0055 & 4.340 & 0.119 & 0.3024 & 0.0055 & 0.663 & 1703 & 27 & 1698 & 38 & 0 \\
\hline 40.1 & 100 & 64 & 0.64 & 22.9 & 0.001101 & 1.77 & 3.743 & 0.056 & 0.1097 & 0.0014 & 0.2625 & 0.0042 & 3.418 & 0.193 & 0.2625 & 0.0042 & 0.285 & 1502 & 22 & 1517 & 102 & 1 \\
\hline 41.1 & 227 & 78 & 0.34 & 55.6 & 0.000016 & 0.02 & 3.508 & 0.041 & 0.0984 & 0.0008 & 0.2852 & 0.0035 & 3.894 & 0.083 & 0.0990 & 0.0013 & 0.827 & 1618 & 18 & 1606 & 24 & -1 \\
\hline 42.1 & 640 & 167 & 0.26 & 94.2 & 0.000016 & 0.03 & 5.836 & 0.063 & 0.0728 & 0.0005 & 0.1713 & 0.0019 & 1.713 & 0.023 & 0.1713 & 0.0019 & 0.817 & 1019 & 10 & 1001 & 15 & -2 \\
\hline 43.1 & 87 & 28 & 0.32 & 15.3 & 0.000178 & 0.30 & 4.897 & 0.071 & 0.0794 & 0.0014 & 0.2045 & 0.0031 & 2.275 & 0.078 & 0.0807 & 0.0021 & 0.698 & 1200 & 17 & 1214 & 51 & 1 \\
\hline 44.1 & 151 & 58 & 0.38 & 20.8 & - & $<0.01$ & 6.234 & 0.082 & 0.0709 & 0.0011 & 0.1604 & 0.0021 & 1.569 & 0.032 & 0.1604 & 0.0021 & 0.645 & 959 & 12 & 955 & 32 & 0 \\
\hline 45.1 & 167 & 66 & 0.40 & 24.2 & 0.000368 & 0.64 & 5.927 & 0.077 & 0.0712 & 0.0011 & 0.1689 & 0.0023 & 1.683 & 0.060 & 0.0722 & 0.0020 & 0.707 & 1006 & 13 & 993 & 56 & -1 \\
\hline 46.1 & 111 & 89 & 0.80 & 24.4 & - & $<0.01$ & 3.899 & 0.052 & 0.0932 & 0.0012 & 0.2565 & 0.0034 & 3.307 & 0.063 & 0.2565 & 0.0034 & 0.710 & 1472 & 18 & 1498 & 25 & 2 \\
\hline 47.1 & 221 & 117 & 0.53 & 58.5 & 0.000017 & 0.03 & 3.247 & 0.038 & 0.1038 & 0.0008 & 0.3081 & 0.0039 & 4.428 & 0.110 & 0.1042 & 0.0016 & 0.837 & 1731 & 19 & 1701 & 29 & -2 \\
\hline 48.1 & 91 & 65 & 0.72 & 23.6 & 0.001061 & 1.69 & 3.305 & 0.047 & 0.1140 & 0.0014 & 0.2976 & 0.0048 & 4.095 & 0.194 & 0.0998 & 0.0036 & 0.769 & 1680 & 24 & 1620 & 68 & -4 \\
\hline 49.1 & 21 & 18 & 0.88 & 3.1 & 0.000855 & 1.51 & 5.630 & 0.138 & 0.0735 & 0.0029 & 0.1749 & 0.0046 & 1.473 & 0.194 & 0.1749 & 0.0046 & 0.199 & 1039 & 25 & 642 & 277 & -62 \\
\hline 50.1 & 284 & 111 & 0.39 & 49.8 & - & $<0.01$ & 4.894 & 0.059 & 0.0787 & 0.0008 & 0.2046 & 0.0026 & 2.249 & 0.059 & 0.0797 & 0.0014 & 0.801 & 1200 & 14 & 1191 & 35 & -1 \\
\hline 51.1 & 434 & 131 & 0.30 & 61.5 & - & $<0.01$ & 6.066 & 0.068 & 0.0705 & 0.0007 & 0.1649 & 0.0019 & 1.604 & 0.023 & 0.1649 & 0.0019 & 0.771 & 984 & 10 & 944 & 19 & -4 \\
\hline 52.1 & 451 & 203 & 0.45 & 103.3 & 0.000009 & 0.01 & 3.748 & 0.041 & 0.0933 & 0.0006 & 0.2669 & 0.0031 & 3.451 & 0.075 & 0.0938 & 0.0012 & 0.858 & 1525 & 16 & 1503 & 25 & -1 \\
\hline 53.1 & 96 & 63 & 0.66 & 22.7 & - & $<0.01$ & 3.610 & 0.051 & 0.0978 & 0.0013 & 0.2770 & 0.0039 & 3.734 & 0.073 & 0.2770 & 0.0039 & 0.724 & 1576 & 20 & 1582 & 25 & 0 \\
\hline 54.1 & 528 & 87 & 0.17 & 134.7 & - & $<0.01$ & 3.369 & 0.037 & 0.1011 & 0.0006 & 0.2969 & 0.0033 & 4.148 & 0.062 & 0.1013 & 0.0007 & 0.886 & 1676 & 16 & 1648 & 13 & -2 \\
\hline 55.1 & 72 & 44 & 0.61 & 16.7 & - & $<0.01$ & 3.688 & 0.056 & 0.0909 & 0.0015 & 0.2720 & 0.0046 & 3.510 & 0.149 & 0.0936 & 0.0030 & 0.750 & 1551 & 23 & 1500 & 60 & -3 \\
\hline 56.1 & 41 & 17 & 0.41 & 6.1 & 0.000240 & 0.42 & 5.764 & 0.128 & 0.0644 & 0.0021 & 0.1735 & 0.0041 & 1.543 & 0.113 & 0.0645 & 0.0039 & 0.656 & 1031 & 23 & 758 & 127 & -36 \\
\hline 57.1 & 218 & 117 & 0.53 & 60.7 & 0.000033 & 0.05 & 3.089 & 0.037 & 0.1096 & 0.0009 & 0.3235 & 0.0039 & 4.871 & 0.072 & 0.3235 & 0.0039 & 0.815 & 1807 & 19 & 1786 & 16 & -1 \\
\hline 58.1 & 39 & 21 & 0.54 & 6.5 & 0.000143 & 0.24 & 5.131 & 0.100 & 0.0801 & 0.0022 & 0.1944 & 0.0038 & 2.092 & 0.078 & 0.1944 & 0.0038 & 0.520 & 1145 & 20 & 1148 & 64 & 0 \\
\hline 59.1 & 78 & 24 & 0.31 & 10.5 & - & $<0.01$ & 6.400 & 0.101 & 0.0731 & 0.0016 & 0.1563 & 0.0025 & 1.575 & 0.043 & 0.1563 & 0.0025 & 0.578 & 936 & 14 & 1017 & 45 & 8 \\
\hline 60.1 & 130 & 109 & 0.84 & 20.8 & 0.000053 & 0.09 & 5.355 & 0.073 & 0.0761 & 0.0012 & 0.1866 & 0.0025 & 1.938 & 0.042 & 0.1866 & 0.0025 & 0.626 & 1103 & 14 & 1077 & 34 & -2 \\
\hline 61.1 & 236 & 148 & 0.63 & 59.3 & - & $<0.01$ & 3.422 & 0.041 & 0.0990 & 0.0008 & 0.2926 & 0.0038 & 4.039 & 0.113 & 0.1001 & 0.0019 & 0.830 & 1654 & 19 & 1626 & 35 & -2 \\
\hline 62.1 & 81 & 27 & 0.33 & 11.5 & - & $<0.01$ & 6.045 & 0.094 & 0.0721 & 0.0016 & 0.1655 & 0.0026 & 1.659 & 0.046 & 0.1655 & 0.0026 & 0.564 & 987 & 14 & 1005 & 46 & 2 \\
\hline 63.1 & 226 & 205 & 0.91 & 61.0 & 0.000036 & 0.06 & 3.182 & 0.039 & 0.1023 & 0.0008 & 0.3149 & 0.0044 & 4.525 & 0.158 & 0.1042 & 0.0025 & 0.848 & 1765 & 22 & 1700 & 44 & -4 \\
\hline 64.1 & 114 & 78 & 0.68 & 16.8 & 0.000985 & 1.69 & 5.864 & 0.082 & 0.0853 & 0.0014 & 0.1683 & 0.0027 & 1.730 & 0.099 & 0.0745 & 0.0035 & 0.736 & 1003 & 15 & 1056 & 94 & 5 \\
\hline 65.1 & 408 & 112 & 0.27 & 99.9 & 0.000038 & 0.06 & 3.509 & 0.039 & 0.0967 & 0.0006 & 0.2856 & 0.0033 & 3.883 & 0.068 & 0.0986 & 0.0010 & 0.860 & 1619 & 16 & 1598 & 18 & -1 \\
\hline
\end{tabular}

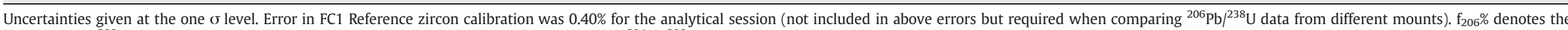
percentage of ${ }^{206} \mathrm{~Pb}$ that is common $\mathrm{Pb}$. Correction for common $\mathrm{Pb}$ made using the measured ${ }^{204} \mathrm{~Pb} /{ }^{206} \mathrm{~Pb}$ ratio. For $\%$ Disc, $0 \%$ denotes a concordant analysis. $\mathrm{Pb}{ }^{*}$ is the proportion of $\mathrm{Pb}$ that is radiogenic 


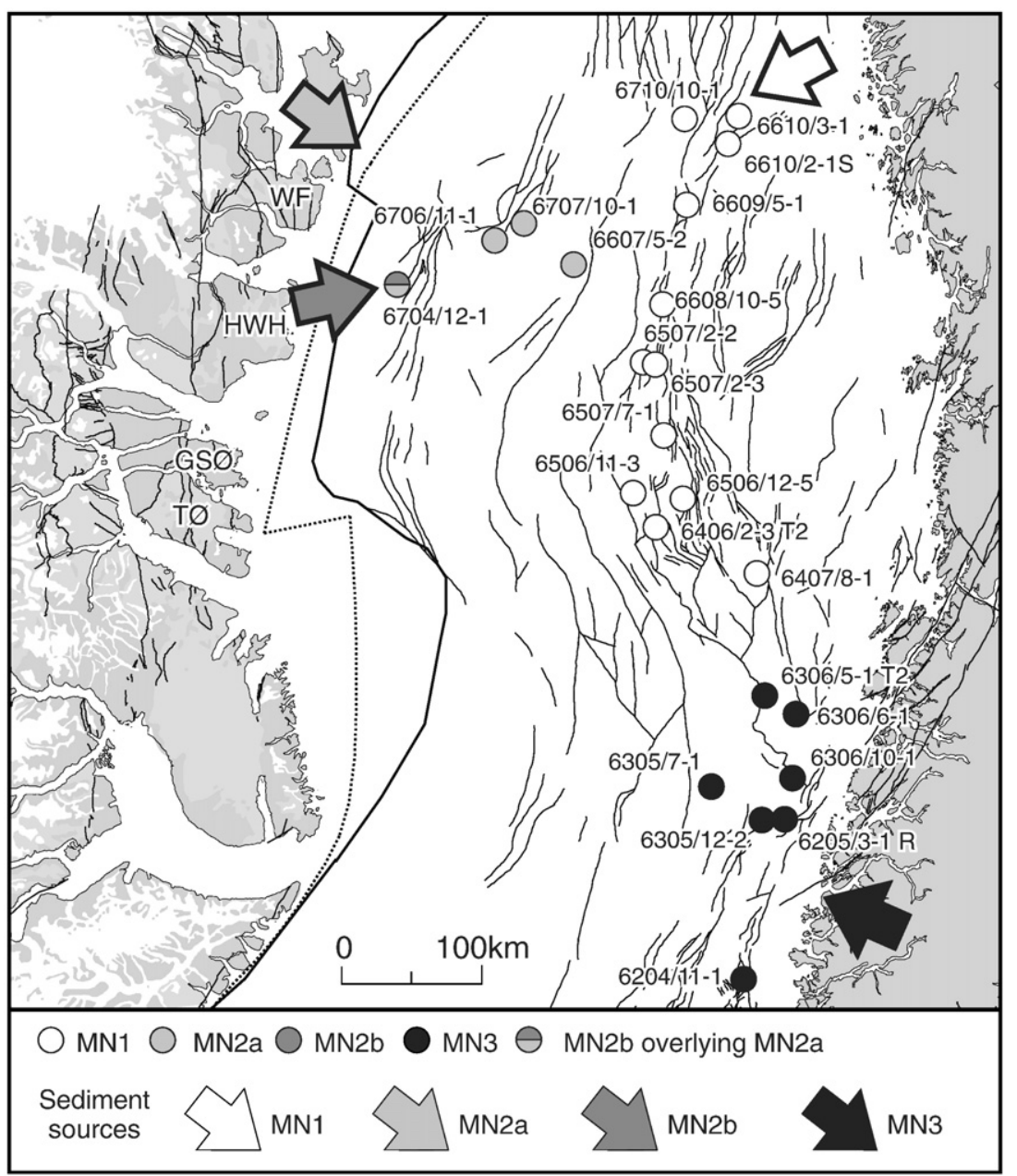

Fig. 5. Regional distribution and inferred sources of Cretaceous-Paleocene sand types MN1, MN2 and MN3 in the Norwegian Sea. Adapted from Morton et al. (2005a).

figures, and especially to BP Norway AS, who funded a large proportion of the analysis. Thanks are also due to Peter Cawood, Hilmar von Eynatten, Emma Rehnström and Andy Whitham for their constructive comments on earlier versions of this manuscript.

\section{References}

Bergman, S., Högdahl, K., Nironen, M., Ogenhall, E., Sjöström, H., Lundqvist, L., Lahtinen, R., 2008. Timing of Palaeoproterozoic intra-orogenic sedimentation in the central Fennoscandian Shield; evidence from detrital zircon in metasandstone. Precambrian Research 161, 231-249.

Bingen, B., Demaiffe, D., van Breemen, O., 1998. The 616 Ma old Egersund basaltic dike swarm, SW Norway, and Late Neoproterozoic opening of the Iapetus Ocean. Journal of Geology 106, 565-574

Bingen, B., Nordgulen, Ø., Solli, A., 2002. U-Pb geochronology of Paleozoic events in the mid-Scandinavian Caledonides (coord.) In: Eide, E.A. (Ed.), BATLAS - Mid Norway plate reconstruction atlas with global and Atlantic perspectives. Geological Survey of Norway, pp. 66-67.

Cawood, P.A., McCausland, P.J.A., Dunning, G.R., 2001. Opening lapetus: constraints from the Laurentian margin in Newfoundland. Bulletin of the Geological Society of America 113, 443-453.

Cawood, P.A., Nemchin, A.A., Smith, M., Loewy, S., 2003. Source of the Dalradian Supergroup constrained by $\mathrm{U}-\mathrm{Pb}$ dating of detrital zircon and implications for the East Laurentian margin. Journal of the Geological Society, London 160, 231-246.

Cawood, P.A., Nemchin, A.A., Strachan, R., Prave, T., Krabbendam, M., 2007. Sedimentary basin and detrital zircon record along East Laurentia and Baltica during assembly and breakup of Rodinia. Journal of the Geological Society, London 164, 257-275.

Claesson, S., Huhma, H., Kinny, P.D., Williams, I.S., 1993. Svecofennian detrital zircon ages - implications for the Precambrian evolution of the Baltic Shield. Precambrian Research 64, 109-130.

Corfu, F., 2004. U-Pb age, setting and tectonic significance of the anorthositemangerite-charnockite-granite suite, Lofoten-Vesterålen, Norway. Journal of Petrology 45, 1799-1819.
Evans, J.A., Chisholm, J.I., Leng, M.J., 2001. How U-Pb detrital monazite ages contribute to the interpretation of the Pennine Basin infill. Journal of the Geological Society, London 158, 741-744

Fedo, C.M., Sircombe, K.N., Rainbird, R.H., 2003. Detrital zircon analysis of the sedimentary record. In: Hanchar, J.M., Hoskin, P.W.O. (Eds.), Zircon. Reviews in Mineralogy and Geochemistry, vol. 53, pp. 277-303.

Fonneland, H.C., Lien, T., Martinsen, O.J., Pedersen, R.B., Kosler, J., 2004. Onshore and offshore provenance studies: a key to understanding the deposition of deepmarine sandstones in the Norwegian Sea. Sedimentary Geology 164, 147-159.

Gaal, G., Gorbatschev, R., 1987. An outline of the Precambrian evolution of the Baltic Shield. Precambrian Research 35, 15-52.

Gower, C.F., Ryan, A.B., Rivers, T., 1991. Mid-Proterozoic Laurentia-Baltica: an overview of its geological evolution and a summary of the contributions made by this volume. In: Gower, C.F., Rivers, T., Ryan, B. (Eds.), Mid-Proterozoic Laurentia-Baltica. Geological Association of Canada, Special Paper, vol. 38, pp. 1-20.

Hemming S.R. Bond, G.C. Broecker, W.S., Sharp, W.D., Klas-Mendelson, M., 2000. Evidence from ${ }^{40} \mathrm{Ar} /{ }^{39} \mathrm{Ar}$ ages of individual hornblende grains for varying Laurentide sources of iceberg discharges 22,000 to $10,500 \mathrm{yr}$ B.P. Quaternary Geology 54, 372-383.

Ireland, T.R., 1992. Crustal evolution of New Zealand: evidence from age distributions of detrital zircons in Western Province paragneisses and Torlesse greywacke. Geochimica et Cosmochimica Acta 56, 911-920.

Jacobsen, S.B., Wasserburg, G.J., 1978. Interpretation of $\mathrm{Nd}, \mathrm{Sr}$ and $\mathrm{Pb}$ isotope data from Archaean migmatites in Lofoten-Vesterålen, Norway. Earth and Planetary Science Letters 41, 245-253.

Kirkland, C.L., Daly, J.S., Whitehouse, M.S., 2007. Provenance and terrane evolution of the Kalak Nappe Complex, Norwegian Caledonides: implications for Neoproterozoic paleogeography and tectonics. Journal of Geology 115, 21-41.

Koistinen, T., Stephens, M.B., Bogatchev, V., Nørdgulen, O., Wennerström, M., Korhonen, J., 2001. Geological map of the Fennoscandian Shield, scale 1: 2000 000. Geologica Surveys of Finland, Norway and Sweden and the North-West Departmnet of Natural Resources of Russia.

Kosler, J. Sylvester, PJ., 2003. Present trends and the future of zircon in geochronology: lase ablation ICPMS, Considerations in Zircon Geochronology by SIMS. In: Hanchar, J.M. Hoskin, P.W.O. (Eds.), Zircon. Reviews in Mineralogy and Geochemistry, 53, pp. 243-275. 
Knudsen, T.-L., Griffin, W.L., Hartz, E.H., Andresen, A., Jackson, S.E., 2001. In-situ hafnium and lead isotope analyses of detrital zircons from the Devonian sedimentary basin of NE Greenland: a record of repeated crustal reworking. Contributions to Mineralogy and Petrology 141, 83-94.

Larson, S.Å., Berglund, J., 1992. A chronological subdivision of the Transscandinavian Igneous Belt - three magmatic episodes? Geologiska Föreningens i Stockholm Förhandlingar 114, 459-461.

Ludwig, K.R., 1999. Isoplot, a geochronological toolkit for Microsoft Excel. Berkeley Geochronology Center, Special Publication 1a.

Ludwig, K.R., 2001. SQUID 1.00, a user's manual. Berkeley Geochronology Center, Special Publication 2.

Mahoney, J.B., Mustard, P.S., Haggart, J.W., Friedman, R.M., Fanning, C.M., McNicoll, V.J. 1999. Archean zircons in Cretaceous strata of the western Canadian Cordillera: the "Baja B.C." hypothesis fails a "crucial test". Geology 27, 195-198.

Morton, A.C., Whitham, A.G., Fanning, C.M., 2005a. Provenance of Late CretaceousPaleocene submarine fan sandstones in the Norwegian Sea: integration of heavy mineral, mineral chemical and zircon age data. Sedimentary Geology 182, 3-28.

Morton, A.C., Whitham, A.G., Fanning, C.M., Claoué-Long, J.C., 2005b. The role of Eas Greenland as a source of sediment to the Vøring Basin during the Late Cretaceous. In: Wandås, B.T.G., Eide, E.A., Gradstein, F., Nystuen, J.P. (Eds.), Onshore-offshore relationships on the North Atlantic margin, vol. 12. Norwegian Petroleum Society Special Publication, pp. 83-110.

Paces, J.B., Miller, J.D., 1993. Precise U-Pb ages of Duluth Complex and related mafic intrusions, northeastern Minnesota: Geochronological insights to physical, petrogenetic, paleomagnetic, and tectonomagmatic process associated with the $1.1 \mathrm{Ga}$ Midcontinent Rift System. Journal of Geophysical Research 98, 13997-14013.

Rehnström, E.F., Corfu, F., 2004. Palaeoproterozoic ages of authochthonous and allochthonous granites from the northern Swedish Caledonides - regional and palaeogeographical implications. Precambrian Research 132, 363-378.

Roberts, D., 2003. The Scandinavian Caledonides: event chronology, paleogeographic settings and likely modern analogues. Tectonophysics 365, 283-299.

Roberts, D., Gee, D.G., 1985. An introduction to the structure of the Scandinavian Caledonides. In: Gee, D.G., Sturt, B. (Eds.), The Caledonian Orogen - Scandinavia and related areas. John Wiley and Son, Chichester, pp. 55-68.

Skår, Ø., 2002. U-Pb geochronology and geochemistry of early Proterozoic rocks of the tectonic basement windows in central Nordland, Caledonides of north-central Norway. Precambrian Research 116, 265-283.
Skår, Ø., Pedersen, R.B., 2003. Relations between granitoid magmatism and migmatization: $\mathrm{U}-\mathrm{Pb}$ geochronological evidence from the Western Gneiss Complex, Norway. Journal of the Geological Society, London 160, 935-946.

Skiöld, T., Rutland, R.W.R., 2006. Successive $\sim 1.94$ Ga plutonism and $\sim 1.92$ Ga deformation and metamorphism south of the Skellefte district, northern Sweden: substantiation of the marginal basin accretion hypothesis of Svecofennian evolution. Precambrian Research 148, 181-204.

Starmer, IC 1996. Accretion, rifting rotation and collision in the North Atlantic supercontinent, 1700-950 Ma. In: Brewer, T.S. (Ed.), Precambrian crustal evolution in the North Atlantic region, vol. 112. Geological Society, London, Special Publication, pp. 219-248.

Tera, F., Wasserburg, G., 1972. U-Th-Pb systematics in three Apollo 14 basalts and the problem of initial $\mathrm{Pb}$ in lunar rocks. Earth and Planetary Science Letters 14, 281-304.

Tucker, R.D., Krogh, T.E., Råheim, A., 1991. Proterozoic evolution and age-province boundaries in the central part of the Western Gneiss Region, Norway: results of U$\mathrm{Pb}$ dating of accessory minerals from Trondheimsfjord to Geiranger. In: Gower, C.F., Rivers, T., Ryan, B. (Eds.), Mid-Proterozoic Laurentia-Baltica, vol. 38. Geological Association of Canada, Special Paper, pp. 149-173.

Von Eynatten, H., Gaupp, R., 1999. Provenance of Cretaceous synorogenic sandstones in the Eastern Alps: constraints from framework petrography, heavy mineral analysis and mineral chemistry. Sedimentary Geology 124, 81-111.

Von Eynatten, H., Gaupp, R., Wijbrans, J.R., 1996. ${ }^{40} \mathrm{Ar} /{ }^{39} \mathrm{Ar}$ laser-probe dating of detrital white micas from Cretaceous sedimentary rocks of the Eastern Alps: evidence for Variscan high-pressure metamorphism and implications for Alpine orogeny. Geology 24, 691-694.

Williams, I.S., 1998. U-Th-Pb geochronology by ion microprobe. In: McKibben, M.A., ShanksIII III, W.C., Ridley, W.I. (Eds.), Applications of microanalytical techniques to understanding mineralising processes. Society of Economic Geologists, Reviews in Economic Geology, vol. 7, pp. 1-35.

Williams, I.S., Claesson, S., 1987. Isotopic evidence for the Precambrian provenance and Caledonian metamorphism of high grade paragneisses from the Seve Nappes, Scandinavian Caledonides. II. Ion microprobe U-Th-Pb. Contributions to Mineralogy and Petrology 97, 205-217.

Yoshinobu, A.S., Barnes, C.G., Nordgulen, Ø., Prestvik, T., Fanning, M., Pedersen, R.B., 2002. Ordovician magmatism, deformation, and exhumation in the Caledonides of central Norway: an orphan of the Taconic orogeny? Geology 30, 883-886. 